University of Louisville

ThinkIR: The University of Louisville's Institutional Repository

Electronic Theses and Dissertations

$12-2014$

\title{
Biomarker discovery of liver diseases using mass spectrometry- based metabolomics.
}

Xue Shi

University of Louisville

Follow this and additional works at: https://ir.library.louisville.edu/etd

Part of the Chemistry Commons, and the Gastroenterology Commons

\section{Recommended Citation}

Shi, Xue, "Biomarker discovery of liver diseases using mass spectrometry-based metabolomics." (2014). Electronic Theses and Dissertations. Paper 1766.

https://doi.org/10.18297/etd/1766

This Doctoral Dissertation is brought to you for free and open access by ThinkIR: The University of Louisville's Institutional Repository. It has been accepted for inclusion in Electronic Theses and Dissertations by an authorized administrator of ThinkIR: The University of Louisville's Institutional Repository. This title appears here courtesy of the author, who has retained all other copyrights. For more information, please contact thinkir@louisville.edu. 


\title{
BIOMARKER DISCOVERY OF LIVER DISEASES USING MASS SPECTROMETRY-BASED METABOLOMICS
}

\section{By}

Xue Shi

\begin{abstract}
A Dissertation
Submitted to the Faculty of the

in Partial Fulfillment of the Requirements

for the Degree of

Doctor of Philosophy

Department of Chemistry

University of Louisville

Louisville, Kentucky
\end{abstract}

College of Arts and Sciences of the University of Louisville

December 2014 



\title{
BIOMARKER DISCOVERY OF LIVER DISEASES USING MASS SPECTROMETRY-BASED METABOLOMICS
}

\author{
By \\ Xue Shi \\ A Dissertation Approved on \\ November 20, 2014 \\ by the following Dissertation Committee: \\ Dissertation Director \\ Dr. Xiang Zhang \\ Dr. Michael Nantz \\ Dr. Richard Baldwin \\ Dr. Walter Watson
}




\section{DEDICATION}

This dissertation is dedicated to my parents, my husband, my son and to all of my family and friends. 


\section{ACKNOWLEDGEMENTS}

I would like to express my deepest gratitude to my advisor, Dr. Xiang Zhang for taking me under his wing and for his continuous support, optimism, and creativity throughout my research career at the University of Louisville. I am deeply grateful for the enthusiasm towards research that Dr. Zhang installed into me and for his guidance and wisdom in directing that energy towards pushing back the scientific envelope.

I want to thank my committee members Dr. Richard P. Baldwin, Dr. Michael H. Nantz, and Dr. Walter H. Watson for their unconditional assistance and discussion involving scientific, professional, and sometimes personal challenges.

I offer my thanks to other faculty and staff members in the Chemistry Department for their support. Specifically, I would like to thanks Mrs. Xinmin Yin for her help on instrumentation and scientific discussion and Mr. Steve Riley for his help in instrument troubleshooting. I am also grateful for the support of Drs. Matthew Cave, Walter H. Watson, Wenke Feng, Seong Ho Kim, Craig J. McClain and Zhanxiang Zhou from School of Medicine.

I also want to thank a number of my fellow research scientists and graduate students who were of great support as friends and colleagues throughout my graduate studies: Xiaoli Wei, Imhoi Koo, Sen Yao, Marina Malovichko, Aiqin Fang, Ye Yang, Tatiana Krivokhizhina, and Ramya Billur. 
All my family members have been very supportive in every aspect of my life. I need to thank my parents for their love and the great education they gave to me. And without their help to take care of my baby here, this dissertation would not been possible. I want to thank my sister and my brother for their support. I want to thank my husband Hanwen Yuan for his love and support and thank my son Oliver Yuan bring me all the joy and happiness. 


\title{
ABSTRACT \\ BIOMARKER DISCOVERY OF LIVER DISEASES USING MASS SPECTROMETRY-BASED METABOLOMICS
}

\author{
Xue Shi
}

November 20, 2014

Metabolomics has emerged as one of the latest of the "-omics" disciplines that can detect metabolite biomarkers in biological samples. The diverse characteristics of metabolites make the analytical platform challenging in metabolomics. Two bioanalytical platforms were developed in this study to investigate metabolite abundance changes under different biological conditions. We first developed a bioanalytical platform that coupled linear trap quadruple - Fourier transform ion cyclotron mass spectrometer (LTQ-FTICR MS) with direct infusion chip-based nano-electrospray ionization (DI-nESI) and applied it to study polychlorinated biphenyls (PCB) effects on non-alcoholic fatty liver disease (NAFLD). We also employed this platform in conjunction with in vivo metabolite deuterium labeling to study whether chronic alcohol exposure disturbs lipid homeostasis by analyzing triacylglycerol regulation alteration in adipose tissue and liver tissue. We further developed a comprehensive two-dimensional gas chromatography time-of-flight mass spectrometry (GC×GC-TOF MS) platform for metabolomic profiling and applied it to study the effects of arsenic exposure in a mouse model of diet-induced fatty liver disease, as well as the effects of Lactobacillus rhamnosus GG on alcoholic fatty liver 
disease (AFLD) in mice. The results of our metabolomics analyses agreed with the results of histological studies and provided molecular level information for further understanding the mechanisms of liver diseases. 


\section{TABLE OF CONTENTS}

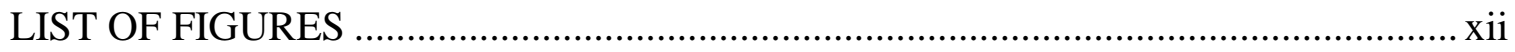

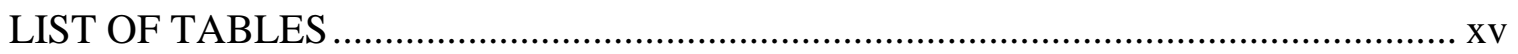

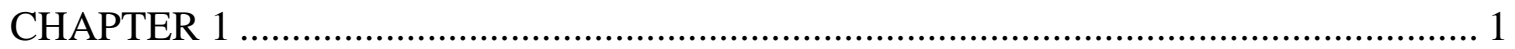

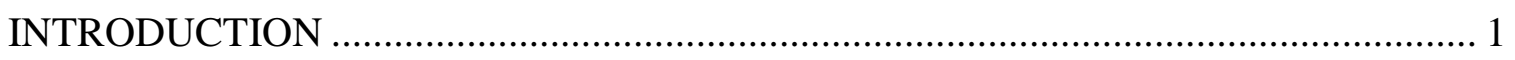

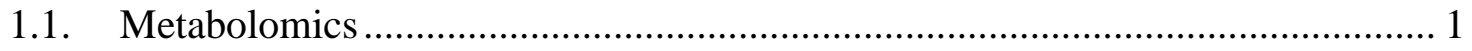

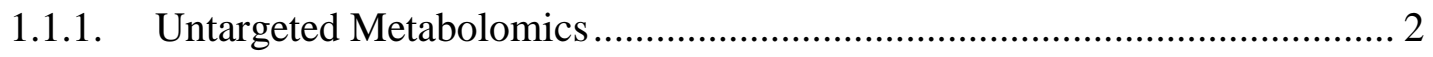

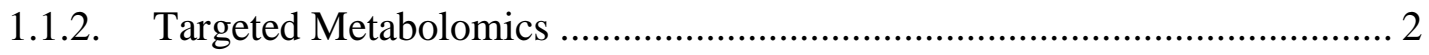

1.1.3. Stable Isotope Assisted Metabolomics (SIAM) ...................................... 3

1.2. Analytical Platforms for Metabolomics ...................................................... 4

1.2.1. Nuclear Magnetic Resonance (NMR) Spectroscopy ................................. 4

1.2.2. Direct Infusion Mass Spectrometry (DI-MS) ........................................ 5

1.2.3. Liquid Chromatography Mass Spectrometry (LC-MS) ............................. 6

1.2.4. Gas Chromatography Mass Spectrometry (GC-MS) ................................ 7

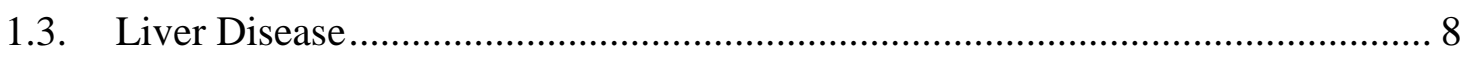

1.4. Objectives of This Dissertation .................................................................. 10

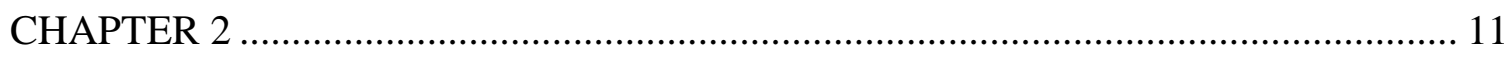

METABOLOMIC ANALYSIS OF THE EFFECTS OF POLYCHLORINATED BIPHENYLS IN NON-ALCOHOLIC LIVER DISEASE .......................................... 11

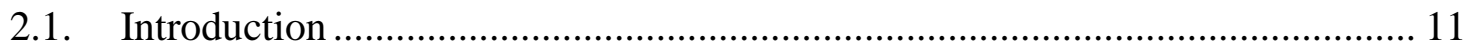

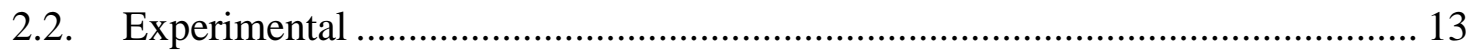

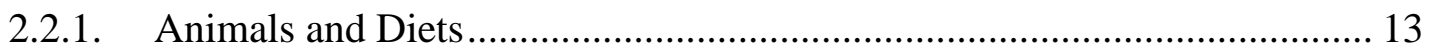

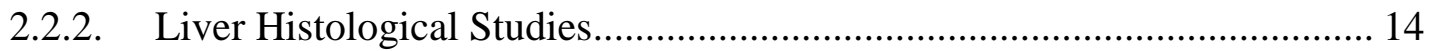

2.2.3. Metabolite Sample Preparation .............................................................. 14

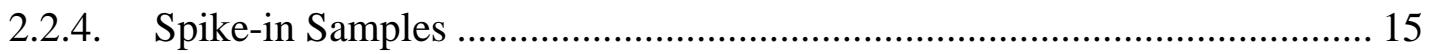

2.2.5. FTICR-MS and LTQ-MS/MS Analysis............................................. 16

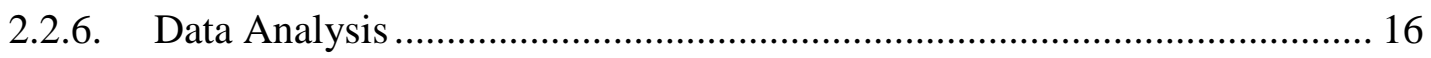




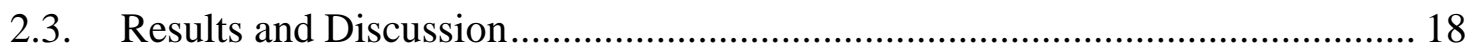

2.3.1. PCB 153 Worsened Steatosis in Mice Fed a High Fat Diet (HFD) ............ 18

2.3.2. Evaluation of Metabolomics Analysis Platform ........................................... 19

2.3.3. Metabolite Identification...................................................................... 22

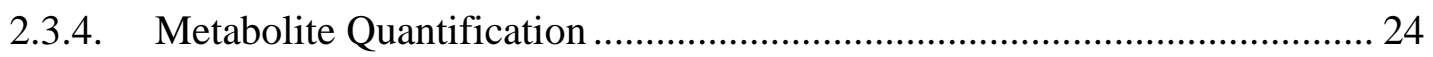

2.3.5. Ingenuity Pathway Analysis (IPA) …………………............................... 32

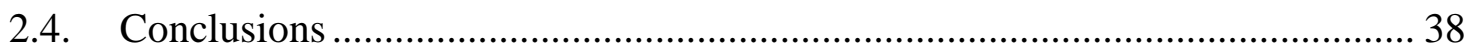

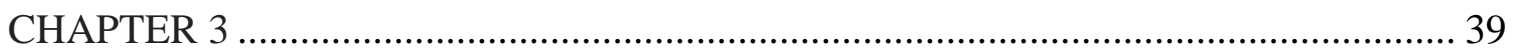

CHRONIC ALCOHOL EXPOSURE DISTURBS LIPID HOMEOSTASIS AT THE ADIPOSE-LIVER AXIS: ANALYSIS OF TRIACYLGLYCEROLS USING HIGH-RESOLUTION MASS SPECTROMETRY IN COMBINATION WITH IN VIVO METABOLITE DEUTERIUM LABELING ……………............................................ 39

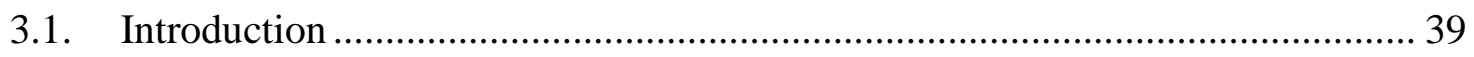

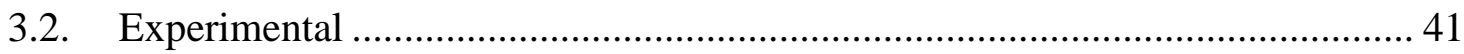

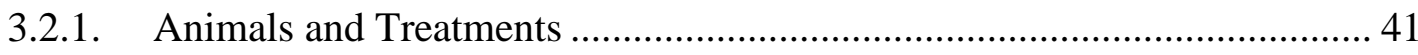

3.2.2. Tissue Sample Preparation........................................................................ 42

3.2.3. FT-MS and LTQ-MS/MS Analysis ........................................................ 43

3.2.4. Metabolite Quantification ............................................................................ 43

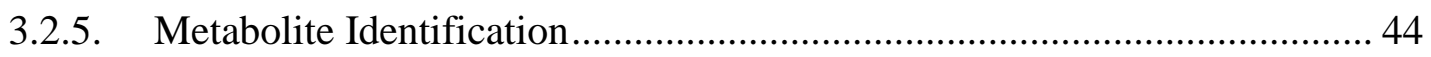

3.2.6. Measurements of Liver Steatosis and Routine Parameters......................... 45

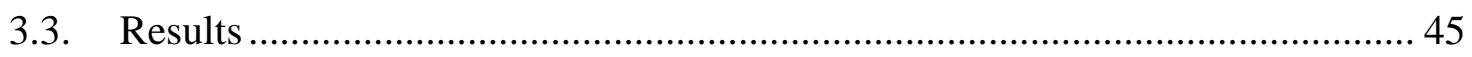

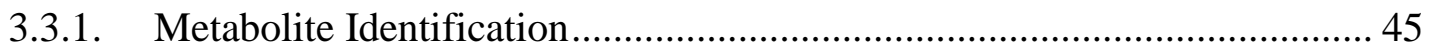

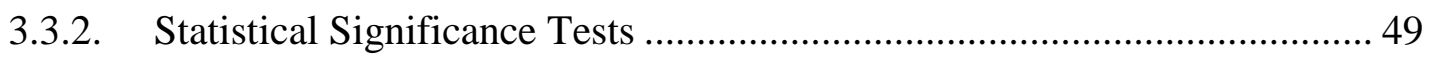

3.3.3. Temporal Analysis ............................................................................... 51

3.3.4. Alterations of Hepatic Neutral Lipid and WAT Mass ................................. 54

3.3.5. Routine Parameters .............................................................................. 57

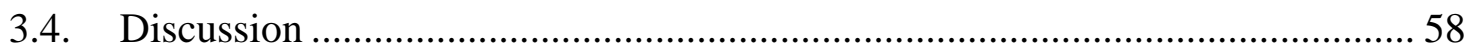

3.4.1. Biological Experiment Design ................................................................. 59

3.4.2. Statistical Significance Tests .................................................................. 59

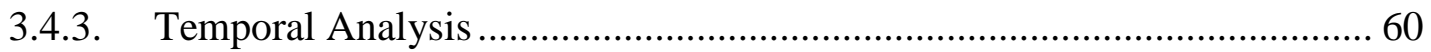

3.4.4. WAT Dysfunction and Fatty Liver .............................................................. 61 


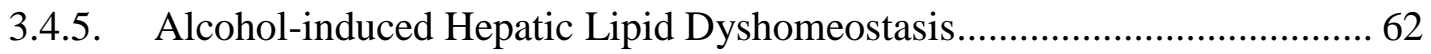

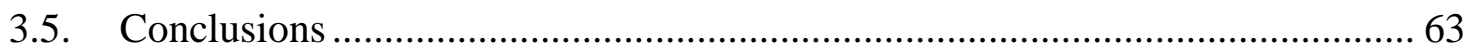

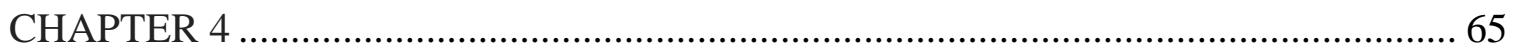

THE EFFECTS OF CHRONIC ARSENIC EXPOSURE IN A MOUSE MODEL OF

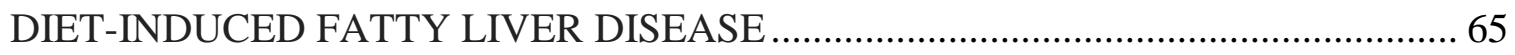

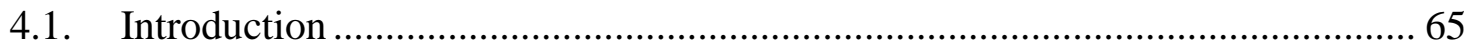

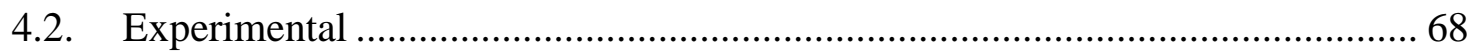

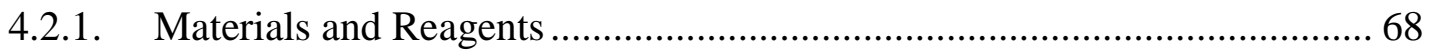

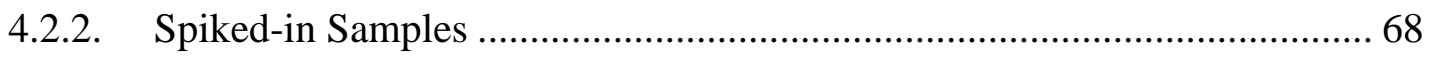

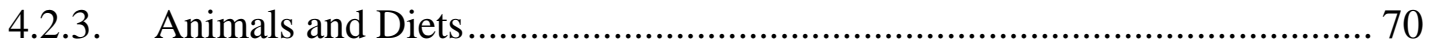

4.2.4. Metabolite Sample Preparation from Mouse Liver ................................ 71

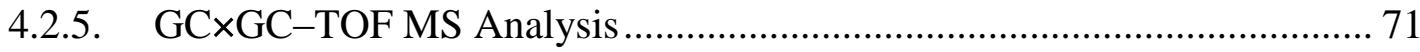

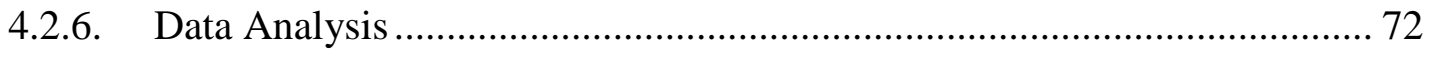

4.2.7. Metabolite Relative Quantification..................................................... 73

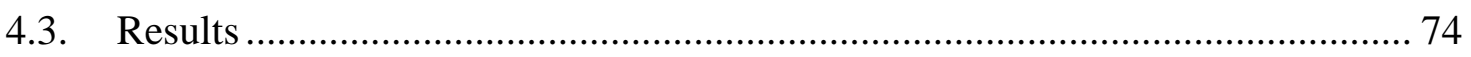

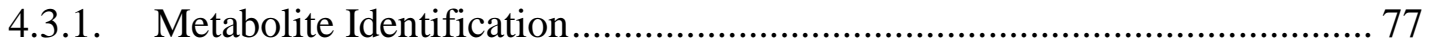

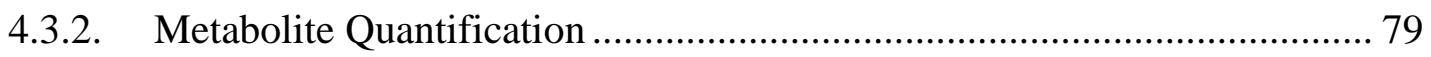

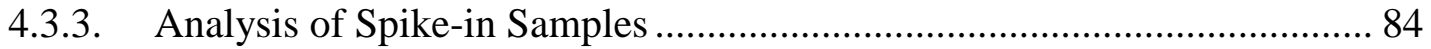

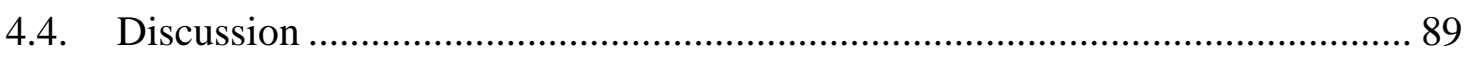

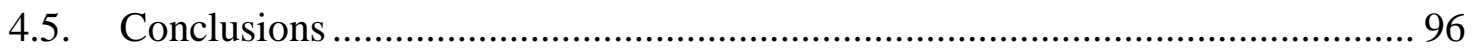

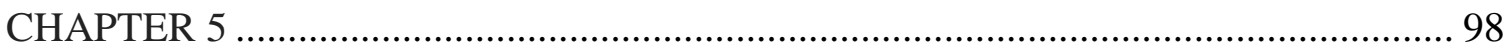

HEPATIC AND FECAL METABOLOMIC ANALYSIS OF THE EFFECTS OF LACTOBACILLUS RHAMNOSUS GG ON ALCOHOLIC FATTY LIVER DISEASE

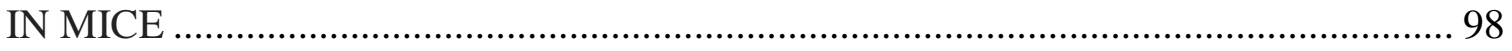

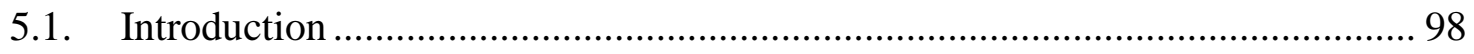

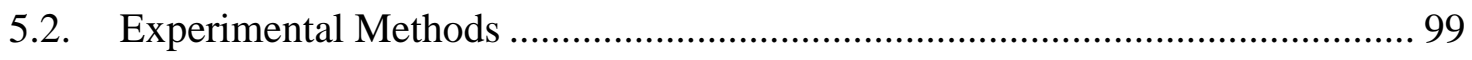

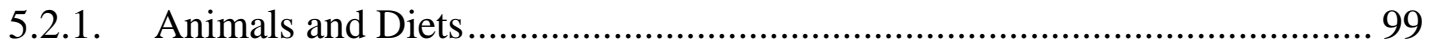

5.2.2. Liver Oil Red O Staining and Liver Total Free Fatty Acid Analysis ....... 100

5.2.3. Metabolite Sample Preparation ........................................................... 100

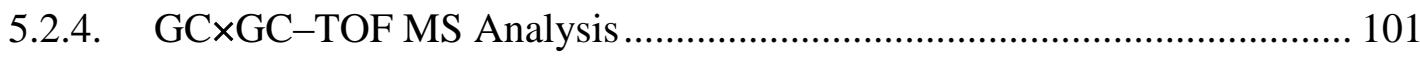

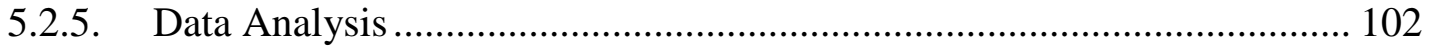




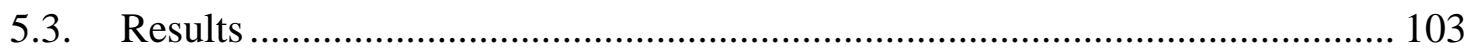

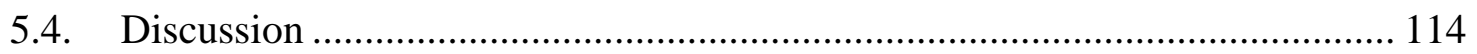

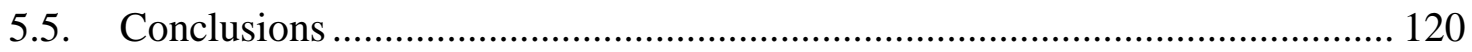

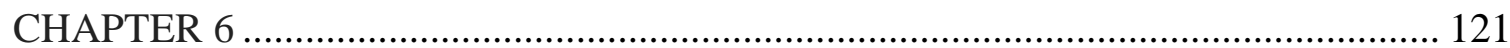

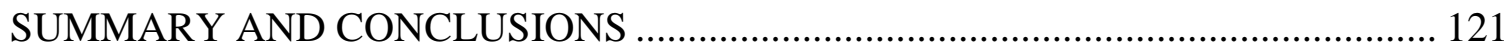

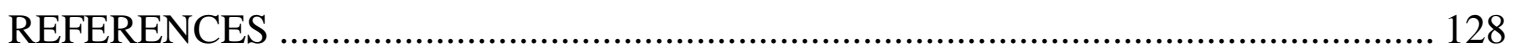

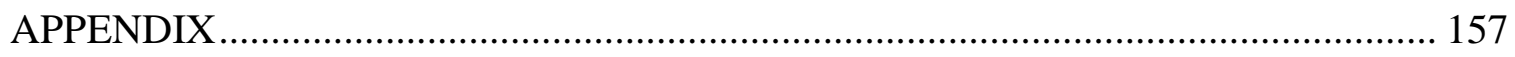

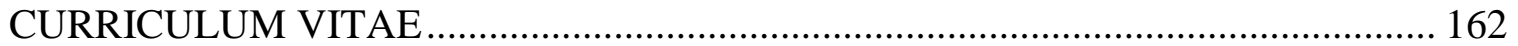




\section{LIST OF FIGURES}

Figure 1. PCB 153 Structure ......................................................................................... 12

Figure 2. PCB 153 worsened hepatic steatosis in mice fed a high fat diet. (A) Oil Red O staining of hepatic sections established the occurrence of micro-vesicular steatosis in the HFD+PCB 153 mice. (B) The same group of mice also showed macro-vesicular steatosis by $\mathrm{H} \&$ E staining.

Figure 3. Relationship between the cutoff value of the p-value of t-test and the values of TPR, PPV, and F1 during the analysis of the spiked-in experiments. (A) TPR (B) PPV (C) F1

Figure 4. An example of identifying metabolites using MS/MS information. The metabolite ion $m / z$ value was measured using FTICR-MS as 156.0773. (A) is the experiment MS/MS spectrum of the metabolite ion. (B) is the matching result of the metabolite between the experiment MS/MS spectrum and the in silico MS/MS spectrum of the same metabolite ion generated by Mass Frontier 7.0. The matched fragment ions are highlighted in red and the unmatched fragment ions in black. (C) is the MS/MS spectrum of the L-histidine standard..

Figure 5. The peak area distribution of metabolite fucose 1-phosphate in the HFD samples (red triangles) and in the HFD+PCB 153 samples (blue stars). The abundance test (pairwise two-tail $t$-test) shows that the regulation of this metabolite in the HFD+PCB 153 group is decreased with a fold change of 2.65 and a $p$-value of $4.84 \times 10^{-4}$.

Figure 6. Erythronic Acid Structure.

Figure 7. IPA proof-of-knowledge characterization of the metabolic networks in mouse liver affected by PCB 153.The dashed line indicates indirect interactions between metabolites while the solid line means a direct metabolite interaction. Filled circles represent metabolites discovered in this work and the color of each filled circle represents the direction and magnitude of fold-changes. Green color means down-regulated, red color means up-regulated.

Figure 8. An example of identifying a deuterium incorporated metabolite using MS/MS information. The metabolite ion $\mathrm{m} / \mathrm{z}$ value was measured on FTICR-MS as 904.74066. (A) is the experimental MS/MS spectrum of non-deuterated metabolite. (B) is the matching result of the non-deuterated metabolite between the experiment MS/MS spectrum and the theoretical MS/MS spectrum generated by Mass Frontier. The matched fragment ions are 
highlighted in red and the not matched ions in black. (C) is the MS/MS spectrum of deuterium incorporated metabolite.

Figure 9. Sample concentration changes of metabolite in two different physiological conditions. The abundance test (pair-wise two-tail t-test) shows that the concentration of this metabolite in the test cohort is increased with a fold change of 2.8 and a p-value of $1.4 \times 10^{-5}$. This metabolite was further identified as TG(16:0/18:2/20:4)[iso6] by MS/MS analysis.

Figure 10. Sample time course trajectories of deuterium labeled triacylglycerols detected in liver, eWAT and sWAT samples.(A) TG(16:0/18:2/20:4)[iso6] with one ${ }^{2} \mathrm{H}$ label and one $\mathrm{Na}^{+}$as adduct ion in liver samples. (B) TG(16:0/16:1/16:1)[iso3] with one ${ }^{2} \mathrm{H}$ label and an adduct ion of $\mathrm{Na}^{+}$eWAT samples. (C) TG(16:0/16:0/18:1)[iso3] with one ${ }^{2} \mathrm{H}$ label and an adduct ion of $\mathrm{Na}^{+}$in sWAT samples 53

Figure 11. Time course trajectory of triacylglycerol TG(16:0/18:2/20:4)[iso6] without any deuterium labeling in liver samples. 53

Figure 12. Time course changes of hepatic lipid content. Hepatic neutral lipid detected by Oil red $\mathrm{O}$ staining of cryostat liver sections. Alcohol exposure increased hepatic neutral lipid (lipid droplets) gradually along the 4 weeks of experiment. 55

Figure 13. Time course changes of WAT tissues.(A) WAT mass. The weights of both eWAT and sWAT in control mice increased gradually during the 4 weeks of experiment. However, the alcohol-fed mice did not show weight change in both eWAT and sWAT at either 2 weeks or 4 weeks. (B) WAT to body weight ratio (\%). Data are expressed as mean \pm SD ( $n=6-8)$. Statistical differences were analyzed by ANOVA followed by Bonferroni post hoc comparison, and means without a common letter differ at $p<0.05 . .56$

Figure 14. Workflow of metabolomics Study by GC×GC-TOF MS. 75

Figure 15. Sample GC $\times$ GC-TOF MS chromatograms of metabolite extract from mouse liver. The $\mathrm{x}$-axis is the first dimension retention time ${ }^{1} t_{R}$ in seconds. The y-axis is the second dimension retention time ${ }^{2} t_{R}$ in seconds. The color bar shows the signal intensity of each peak plotted on the chromatogram in total ion current.

Figure 16. Abundance distribution of metabolite glycine in the samples of HFD+As group and HFD group. The abundance test (pairwise two-tail t-test) shows that the regulation of this metabolite in the HFD+As group is decreased with a fold change of 1.7 and a $\mathrm{p}$-value of $5.0 \times 10^{-3}$ comparing with HFD group.

Figure 17. Boxplot of peak area of the 28 spiked-in metabolites in G10 before reference spectrum-based peak area conversion (A) and after the reference spectrum-based peak area conversion (B). + refers to the values of peak area considered as outliers.

Figure 18. The receiver operating characteristic (ROC) curve of the spike-in data. The area under curve (AUC) of the three comparisons is $0.87,0.71$, and 0.81 for G20 vs. G10, G40 vs. G10 and G40 vs. G20, respectively. 88

Figure 19. Clustering analysis of the four sample groups using fuzzy $\mathrm{C}$ mean algorithm. The input data were the abundance of all metabolites that were detected with significant 
regulation changes between two or more sample groups when the FDR threshold $\mathrm{q} \leq 0.2$. (A) depicts the clustering results of all four sample groups. The overall clustering accuracy reached 0.70 . (B) displays the clustering accuracy between two sample groups.

Figure 20. Sample GC $\times$ GC-TOF MS chromatogram of metabolite extract from mouse fecal samples. The $x$-axis is the first dimension retention time ${ }^{1} t_{R}$ in seconds. The $y$-axis is the second dimension retention time ${ }^{2} t_{R}$ in seconds. The color bar shows the signal intensity of each peak plotted on the chromatogram in total ion current......................... 105

Figure 21. Abundance distribution of metabolite hexanoic acid in four sample groups. (A) is in liver samples, and (B) is in fecal samples......................................................... 108

Figure 22. Effect of LGGs on hepatic free fatty acid levels. Stars * refer to the amount of total free fatty acids between two sample groups is significantly different with $p<0.05$.

Figure 23. Effect of LGGs on liver fat accumulation. Hepatic cryosections were Oil red O stained and images were acquired by light microscopy 114

Figure 24. Clustering of metabolite profiles using partial least squares discriminant analysis (PLSDA). (A) is the clustering result of metabolite profiles of all liver samples and (B) is the clustering result of all fecal samples. 


\section{LIST OF TABLES}

Table 1. Metabolites detected with significant abundance changes between the HFD

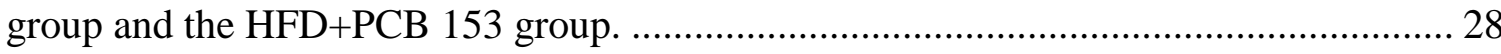

Table 2. Metabolites detected with significant abundance changes between the CD+PCB 153 group and the HFD+PCB 153 group. 30

Table 3. Metabolites detected with significant abundance changes between the CD group and the HFD group...... 31

Table 4. List of triacylglycerols in liver identified with significant concentration changes between the control cohort and the test cohort at two and four weeks.

Table 5. List of triacylglycerols in sWAT and eWAT identified with significant concentration changes between the control cohort and the test cohort at two and four weeks

Table 6. Body weight, liver weight and plasma parameters of the control cohort and the test cohort at two and four weeks.

Table 7. Metabolites with significance abundance difference between sample groups HFD and LFD.

Table 8. Metabolites with significance abundance difference between sample groups LFD+As and LFD.

Table 9. Metabolites with significance abundance difference between sample groups HFD+As and HFD.

Table 10. The number of metabolites with significant differences between sample groups with different values of FDR threshold. It should be noted that the number of metabolites detected with significant difference between sample groups are different from these listed in Tables 7, 8, 9. The number of metabolites listed in this table was directly calculated from the alignment table. That is the identifications of these compounds were not yet confirmed using the methods described in section 4.3.1.

Table 11. Metabolites with significant changes of abundance level among the four liver sample groups.

Table 12. Metabolites with significant change of abundance level among four fecal sample groups. 


\section{CHAPTER 1 INTRODUCTION}

\subsection{Metabolomics}

Metabolomics is the study of biochemical process involving small-molecule metabolites in a biological system. It aims to simultaneously measure and interpret the complex time-related concentration, activity, and flux of all metabolites present in metabolome. In contrast to classical biochemical approaches that often focus on a single metabolite, metabolomics reveals biochemical activities of a broad range of small molecules such as lipids, amino acids, sterols, organic acids, carbohydrates, and vitamins and as such provides a comprehensive overview of the impact of the pathophysiological processes or pharmacological interventions of interest on metabolism and metabolic dynamics[1].

Even though one still does not know how many types of metabolites are present in the metabolome, the human metabolome database (HMDB) has collected 41,808 metabolite entries[2]. Metabolites have a wide range of molecular weights and a large variation in concentration. Metabolites can be polar or nonpolar, as well as organic or inorganic molecules. Also the metabolome is much more dynamic than proteome and genome, which makes the metabolome more time sensitive. The large number of metabolites and diverse characteristics of metabolites make the chemical separation the most challenging technical step in metabolomics. 
With the extreme complexity of metabolome, metabolomics becomes an interdisciplinary field of science, which combines biology, chemistry, mathematics, computer science, statistics with biomedical sciences. Metabolomics studies can be divided into three analytical approaches: untargeted metabolomics, targeted metabolomics, and stable isotope assisted metabolomics.

\subsubsection{Untargeted Metabolomics}

Untargeted metabolomics intends the comprehensive analysis of all measurable metabolites in a sample, such as tissues (e.g., liver, heart, lung, muscle, kidney), biofluids (e.g., blood, serum ,plasma, urine, bile and saliva), cell lines, feces or volatile organic compounds (e.g., those found in breath). This approach is mainly used to investigate the regulation alteration of all detectable metabolites in metabolome. A suite of bioinformatics tools are then employed to deconvolute the instrumental data, perform metabolite identification, quantification, network association analysis and pathway analysis[3-5]. Untargeted metabolomics is mainly used for metabolite biomarker discovery, disease diagnosis, indication of the disease mechanisms, and monitoring responses to therapy, etc.

\subsubsection{Targeted Metabolomics}

Targeted metabolomics measures predefined groups of chemically characterized and biochemically annotated metabolites. It is often used to determine relative abundance and concentrations of a limited number of known metabolites. By analyzing the known metabolites, predefined metabolite-specific signals are measured in biological samples, 
using selected reaction monitoring (SRM) by tandem mass spectrometry (MS/MS)[6], or selected ion recording (SIR) by gas chromatography mass spectrometry (GC-MS)[7].

\subsubsection{Stable Isotope Assisted Metabolomics (SIAM)}

All metabolites are associated with each other in the metabolome to form a large network of molecular reactions. The outputs from one enzymatic biochemical reaction are inputs to another. If multiple pathways result in the same product metabolite, the measured abundance of this metabolite by untargeted metabolic profiling or targeted metabolomics will be the summed abundance of this metabolite in all metabolic pathways. Therefore, the abundance measurement alone is frequently insufficient to determine whether and where a dysregulation took place, and the importance of the individual roles may be misinterpreted or lost.

SIAM uses stable isotope tracers (e.g. ${ }^{13} \mathrm{C}$ or ${ }^{15} \mathrm{~N}$ ) to support studies of biochemical mechanisms[8-10]. For example, when a primary substrate such as ${ }^{13} \mathrm{C}$-glucose is added to a biological system, the relative abundances of formation of particular isotopologues (metabolites that differ only in isotopic composition) of metabolic intermediates from

${ }^{13} \mathrm{C}$-labeled precursors in living cells provide information on the routes taken by the initial ${ }^{13} \mathrm{C}$-atoms. The unique isotopologue patterns of metabolic products reflecting the biosynthetic history of the metabolite under study, i.e., SIAM follows the fate of each atom and its incorporation into a multitude of metabolites produced from the labeled tracer and therefore helps identify and quantify stable isotope labeled molecules and leads to exact biochemical pathway assignment.

While SIAM is able to differentiate the biochemical source of a metabolite, the coverage of metabolites by SIAM is limited to the precursor molecule(s). The metabolites that do 
not inherent labeled atoms, i.e., heavy atoms, from the precursor molecules will not be studied. Another disadvantage of SIAM is that it requires much improved bioinformatics tools to decipher the biochemical information from the experimental data. For example, analysis of unlabeled samples have to be performed to limit the search space of metabolite identification; two dimensional nuclear magnetic resonance (NMR) data have to be used to determine the positions of labeled atoms in a metabolite. The low sensitivity of NMR induces a significant number of isotopologues without chemical structure information.

\subsection{Analytical Platforms for Metabolomics}

Owing to the significant complexity of the metabolome, there is nosingle instrument that can analyze all metabolites simultaneously. Several types of instruments have been utilized to analyze metabolites, including nuclear magnetic magnetic resonance spectroscopy(NMR)[11-17], gas chromatography mass spectrometry GC-MS[18-25] and liquid chromatography mass spectrometry (LC-MS)[26-29]. Each type of instrumental analysis affords limited coverage of the metabolites and therefore, only provides a partial metabolite profile of each sample. The development of comprehensive analytical platforms by integrating the modern instrumental analytical approaches has unraveled the ideal outcomes in metabolomics, and is beneficial to increase the coverage of metabolites that cannot be achieved by a single analytical platform[30].

\subsubsection{Nuclear Magnetic Resonance (NMR) Spectroscopy}

Nuclear magnetic resonance spectroscopy is a technique that exploits magnetic properties of certain atomic nuclei. It determines the physical and chemical properties of atoms in 
each molecule. Suitable samples range from small compounds analyzed with one dimensional proton or ${ }^{13} \mathrm{C}$ NMR spectroscopy to large proteins or nucleic acids using even four dimensional techniques. NMR spectra are unique and often highly predictable for small molecules. Different functional groups are distinguishable and even identical functional groups with differing neighboring substituents still give distinguishable signals. As one of the most common spectroscopic techniques, NMR can uniquely identify and simultaneously quantify a wide range of organic compounds.

NMR-based metabolomics is able to provide a 'holistic view' of the metabolites under certain conditions, and thus is well-suited and advantageous for metabolomic studies[11-17]. Compared with other techniques, NMR-based metabolomics is able to measure intact biomaterials nondestructively and provides rich molecular structural information as well as quantitative information of each detected metabolite. NMR also has good reproducibility with simple sample preparation. However, the sensitivity of NMR is relatively poor compared with MS methods, resulting in metabolites with low abundance not detectable. In case of metabolite biomarker discovery, concentrations of potential biomarkers may be below the detection limit of NMR and these metabolite biomarkers will be missed[30]. Therefore, NMR is inappropriate for the analysis of large number of low-abundance metabolites.

\subsubsection{Direct Infusion Mass Spectrometry (DI-MS)}

Mass spectrometer is a molecular detector as well as a separation device. It measures the mass-to-charge $(\mathrm{m} / \mathrm{z})$ ratio and abundance of gas-phase ions to help identify the type and the amount of species present in a sample[31]. Mass spectrometry has become the 
technique of choice in metabolomics because of its high sensitivity, high accuracy and wide range of covered metabolites[32].

DI-MS directly analyzes metabolites on a high resolution, high accuracy mass spectrometer without any previous separation. A typical analytical platform involves coupling a chip-based nanoelectrospray ion source (nESI) with a Fourier transform ion cyclotron resonance mass spectrometer (FTICR-MS). DI-MS has been used for metabolomics fingerprinting, such as analysis of urine[33], plasma[34] and plant extract[35]. DI-MS has the advantages of high sensitivity and accuracy, large dynamic range, capable of identifying and quantifying metabolites from complex samples, no sample cross-contamination, unchanging sample matrix and high reproducibility[36-42]. The significant drawbacks of DI-MS include severe ion suppression and low ionization efficiencies owing to no pre-separation.

\subsubsection{Liquid Chromatography Mass Spectrometry (LC-MS)}

LC-MS is currently the most widely used analytical platform in metabolomics. It allows for the collection of both quantitative and structural information of each metabolite, and can achieve pg $\mathrm{mL}^{-1}$ sensitivity, Therefore, LC-MS offers a very versatile tool for metabolic study[43]. The LC-MS based metabolomics has been applied to study serum[44,45], liver[46], lung[47] and other diseases.

The choice of LC column and mobile phase composition are the two important components for optimization of a LC-MS system. While most of the LC-MS analyses use reverse phase liquid chromatography (RP-LC) to separate metabolites before MS analysis[48], hydrophobic interaction liquid chromatography (HILIC) has emerged as another choice of separation platform for LC-MS based metabolomics[49]. HILIC is 
similar to normal phase $\mathrm{LC}$, in that the stationary phase is polar; however, in contrast to normal phase LC, the mobile phase in HILIC uses water miscible solvents, such as acetonitrile or methanol, and also contains a substantial amount of water.

To improve the coverage of metabolites, two-dimensional liquid chromatography mass spectrometry (2D LC-MS) has been studied. Some applications combined a HILIC column with a reverse phase column to obtain maximum coverage of the metabolome[50]. Strong cation exchange (SCX) has also been combined with HILIC to get a better separation for water soluble compounds[51].

Coupling LC separation with MS detection leads toimproved MS sensitivity and signal reproducibility by reducing sample complexity, thereby alleviating matrix interferences in the ionization process. Also good separation will result in better quality MS data due to reduced background noise, which will significantly benefits the downstream data analysis for metabolite identification and quantification[52-53].

\subsubsection{Gas Chromatography Mass Spectrometry (GC-MS)}

In GC-MS based metabolomics, metabolites are first separated on a GC column and then subjected to mass spectrometry. GC separates molecules based on their partition coefficients between the mobile phase and stationary phase. The stationary phase of GC column can be categorized as polar, semi-nonpolar and nonpolar. The mobile phase in $\mathrm{GC}$ is usually $\mathrm{He}$ or $\mathrm{N}_{2}$. A GC column is usually coupled to a quadropole or time-of-flight mass spectrometer equipped with a chemical ionization or electron ionization source.

GC-MS has been considered as a traditional and standard approach for metabolomics studies because of its high separation ability, high sensitivity and easy analyte 
identification[54]. It has been widely used for metabolite profiling[55] and biomarker discovery[56]. However, GC-MS based metabolomics requires that all metabolites must be first derivatized and the derivatized metabolites must be volatile and thermally stable. The added metabolite derivatization step introduces a certain level of variation to the GC-MS analysis. For example, incomplete derivatization not only increases the sample complexity by introducing multiple chromatographic peaks, but also makes accurate metabolite identification and quantification challenging or even impossible.

\subsection{Liver Disease}

The liver is the largest internal organ and largest gland in the human body. It plays an important role in many functions, from protein production and blood clotting to cholesterol, glucose and iron metabolism. Liver disease is a type of damage to liver, also called hepatic disease. Symptoms of liver diseases include weakness and fatigue, weight loss, nausea, vomiting, and a yellow discoloration of the skin.

Fatty liver disease (FLD) occurs when fat makes up more than 5\%-10\% of the weight of liver. Even though FLD is reversible, it may lead to inflammatory disease such as steatohepatitis and eventually cirrhosis[57]. FLD can occur after drinking large amount of alcohol(acute), or after a period of heavy drinking(chronic). Centers for Disease Control and Prevention (CDC) defines heavy drinking as consuming 15 drinks or more per week for men 8 drinks for women. Europe is the heaviest drinking region in the world, in terms of the prevalence of excessive alcohol consumption, with over $20 \%$ of the European population aged above 15 reporting heavy drinking[58]. Usally of all chronic heavey drinkers, about $15-20 \%$ develop hepatitis or cirrhosis. Alcoholic liver disease (ALD) 
includes any hepatic manifestation of alcohol overconsumption, including fatty liver disease, alcoholic hepatitis, and cirrhosis.

Ludwig reported a number of patients who had a liver histology characterized by fat accumulation and the presence of hepatic inflammation, with most of the cases presenting fibrosis in the absence of a history of excessive alcohol intake. Therefore, he coined the term "non-alcoholic steatohepatitis"[59,60]. Non-alcoholic fatty liver disease(NAFLD) is now the most common cause of chronic liver disease in the U.S. NAFLD consists of two clinicopathological entities: simple steatosis and non-alcoholic steatohepatitis (NASH). Simple steatosis usually follows a benign non-progressive clinical course. NASH may progress to cirrhosis. These people usually have high cholesterol or triglycerides and diabetes or prediabetes. There are about $10-20 \%$ of NASH among all NAFLD. Potential causes of fatty liver disease include obesity, type 2 diabetes mellitus, dyslipidemia, metabolic syndrome, medications, malnutrition, autoimmune or inherited liver disease, rapid weight loss. NAFLD usually related to insulin resistance and the metabolic syndrome and may also respond to treatments originally developed for other insulin resistant states such as weight loss. So there could be a vicious cycle involving these diseases.

Several efforts have been devoted to liver disease to discover metabolite biomarkers using different metabolomics approaches such as GC-MS[61-63] and HPLC-MS[64-67]. The molecular mechanisms of liver disease are still not fully understood. 


\subsection{Objectives of This Dissertation}

The objectives of this dissertation were to develop a suite of bioanalytical platforms and related bioinformatics tools for the study of metabolite regulation changes under different physiological conditions, and to further apply the developed bioanalytical platforms to discover metabolite biomarkers for various liver diseases. The remaining parts of this dissertation are organized as follows: Chapter two introduces a bioanalytical platform of direct infusion coupled with high resolution mass spectrometry (DI-MS) for global metabolic profiling and its application to study polychlorinated biphenyls (PCB) effects on non-alcoholic fatty liver disease (NAFLD). Chapter Three describes a time course study to investigate how chronic alcohol exposure disturbs lipid homeostasis using DI-MS based metabolomics, where in vivo metabolite deuterium labeling was employed to analyze triacylglycerol in adipose tissue and liver tissue. Chapter Four presents a comprehensive two-dimensional gas chromatography time-of-flight mass spectrometry (GCxGC-TOF MS) system and its application to study the effects of arsenic exposure in a mouse model of diet-induced fatty liver disease. Chapter Five introduces the application of GCxGC-TOF MS platform to study the effects of Lactobacillus rhamnosus GG on alcoholic fatty Liver disease (AFLD) in mice. 


\section{CHAPTER 2 METABOLOMIC ANALYSIS OF THE EFFECTS OF POLYCHLORINATED BIPHENYLS IN NON-ALCOHOLIC LIVER DISEASE}

\subsection{Introduction}

The burden of liver disease has increased in the United States in parallel with the obesity epidemic. The most common liver problems, nonalcoholic fatty liver disease (NAFLD) or its more advanced form, non-alcoholic steatohepatitis (NASH), are thought to be due to overweight/obesity. Polychlorinated biphenyls (PCBs) are persistent environmental pollutants. Exposures to PCBs have been associated with NAFLD in epidemiologic studies[68]. Among PCB congeners, PCB 153 (Figure 1) is present at the highest levels[69]. Dr.Cave's group has been demonstrated that chronic PCB 153 exposure worsened obesity/NAFLD in mice fed a high fat diet (HFD), but had no pathologic effect in mice fed control diet (CD)[70]. To better understand the biochemical mechanisms underlying these observations, a comprehensive investigation using high throughput analysis such as metabolomics is necessary. 


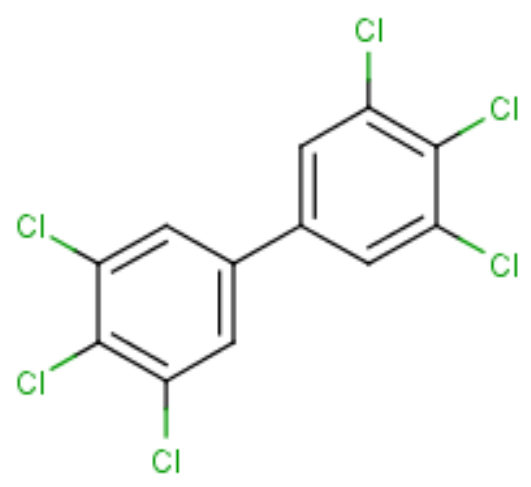

Figure 1. PCB 153 Structure

Metabolomics is the study of low molecular weight molecules (i.e., metabolites) found within cells and biological systems. Due to the extremely diverse chemical characteristics of metabolites, several types of instruments have been utilized to analyze metabolites including nuclear magnetic resonance (NMR)[13,16], liquid chromatography mass spectrometry (LC-MS) and gas chromatography mass spectrometry (GC-MS)[22, 28,71]. Each type of instrumental analysis affords limited coverage of the metabolites, and therefore only provides a partial metabolite profile of each sample. With the advantages of high sensitivity and accuracy, wide dynamic range, and the ability to identify metabolites from complex sample[39,62,72], high-resolution mass spectrometry, such as Fourier transform ion cyclotron resonance mass spectrometer (FTICR-MS), is an attractive option in metabolomics research[73,74].

The objective of this study was to determine if PCB 153 induces NAFLD in mice fed a control diet and/or alters the metabolite profile, and/or exacerbates NAFLD in mice fed a high fat diet. C57BL/6J mice were fed either a control diet (normal chow, CD) or 43\% milk fat diet (high fat diet, HFD) for 12 weeks with or without PCB 153 co-exposure. At 
the end of the feeding period, the mice were anesthetized and liver samples were collected. The metabolite extracts from mouse livers were analyzed using linear trap quadruple - Fourier transform ion cyclotron resonance mass spectrometer (LTQ-FTICR MS) via direct infusion chip-based nano-electrospray ionization (DI-nESI) - mass spectrometry.

\subsection{Experimental}

\subsubsection{Animals and Diets}

The animal experiment were carried out at the School of Medicine, University of Louisville, and the animal protocol was approved by the University of Louisville Institutional Animal Care and Use Committee. Male C57BL/6J mice (8 weeks old, $\mathrm{n}=40$; The Jackson Laboratory, Bar Harbor, Maine) were divided into 4 study groups $(\mathrm{n}=10$ per group) based on diet and PCB 153 exposure in this 12 -week study utilizing a $2 \times 2$ design. The control diet (CD) was the Autoclavable Rodent Diet 5010 from LabDiet, Lebanon, IN; and it consisted of $12.7 \%$ kcal from fat, $58.5 \%$ carbohydrate, and $28.7 \%$ protein from both animal and vegetable sources. The high fat diet (HFD) was TD.88137 from Harlan Laboratories, Madison, WI; and it consisted of $42.7 \%$ kcal from fat (milk fat), $42.0 \%$ carbohydrate (sucrose and corn starch), and 15.2\% protein (casein). PCB 153 (Ultra Scientific, North Kingstown, RI) was administered in corn oil (vehicle) by i.p. injection ( $v s$. corn oil alone) at a dose of $50 \mathrm{mg} / \mathrm{kg}$ on weeks $4,6,8$, and 10 . Mice were housed in a temperature- and light-controlled room (12 h light; $12 \mathrm{~h}$ dark) with food and water ad libitum. The animals were euthanized (sodium pentobarbital, $40 \mathrm{mg} / \mathrm{kg}$ body 
weight, i.p.) at the end of week 12. Thus, four different treatment groups were evaluated in this fashion: CD, CD+PCB 153, HFD, HFD+PCB 153.

\subsubsection{Liver Histological Studies}

Liver sections were frozen using optimal cutting temperature (OCT), a liquid embedding medium or fixed in $10 \%$ buffered formalin for $24 \mathrm{~h}$ and embedded in paraffin for histological examinations. Tissue sections were stained with either Oil Red O (frozen

OCT), hematoxylin-eosin (H\&E; formalin-fixed) or Sirius red stain (formalin-fixed) and examined under light microscopy at $200 \times$ magnification. Photomicrographs were captured using a Nikon Eclipse E600 Microscope.

\subsubsection{Metabolite Sample Preparation}

Metabolites were extracted based on Bligh \& Dyer's method to cover both the polar and non-polar metabolites[75]. Each sample of liver tissue was weighed and homogenized for $2 \mathrm{~min}$ after adding water at a concentration of $100 \mathrm{mg}$ liver tissue $/ \mathrm{mL}$ water. The homogenized sample was then stored at $-80{ }^{\circ} \mathrm{C}$ until use. To extract metabolites from the homogenized liver tissue, $100 \mu \mathrm{L}$ of homogenized liver tissue, $300 \mu \mathrm{L}$ of water, and 1.5 $\mathrm{mL}$ of chloroform-methanol $(\mathrm{v} / \mathrm{v}=1: 2)$ were mixed in a glass tube and vortexed for 1 min, followed by adding $0.5 \mathrm{~mL}$ chloroform, vortexing $1 \mathrm{~min}$, adding $0.5 \mathrm{~mL}$ water and vortexing for $1 \mathrm{~min}$. The mixture was then separated by centrifuging at room temperature for $8 \mathrm{~min}$ at $1100 \mathrm{rpm} .400 \mu \mathrm{L}$ of the organic phase (bottom) were aspirated into another glass tube and dried under nitrogen. Each of the dried samples was dissolved in $100 \mu \mathrm{L}$ of chloroform-methanol $(\mathrm{v} / \mathrm{v}=1: 1)$ and further diluted 25 times before analysis. The aqueous phase was dried in a SpeedVac at $4{ }^{\circ} \mathrm{C}$ to remove methanol, followed by freeze 
drying to vaporize water. Each of the dried water phase samples was re-dissolved in 100 $\mu \mathrm{L}$ methanol and then further diluted 5 times before analysis.

\subsubsection{Spike-in Samples}

About $180 \mathrm{mg}$ of liver tissue from three mice was mixed with deionized water at a concentration of $100 \mathrm{mg} / \mathrm{mL}$. The mixture was then homogenized for 2 min and stored at $-80{ }^{\circ} \mathrm{C}$ until use. To extract metabolites from liver, a $200 \mu \mathrm{L}$ of homogenized liver sample was mixed with $1.6 \mathrm{~mL}$ of methanol and vortexed for $1 \mathrm{~min}$, followed by centrifugation at $4{ }^{\circ} \mathrm{C}$ for $10 \mathrm{~min}$ at $15,000 \mathrm{rpm} .1 .4 \mathrm{~mL}$ of the top solution was aspirated into a plastic tube and dried by $\mathrm{N}_{2}$ flow. After dissolving the dried sample with $200 \mu \mathrm{L}$ of methanol, a stock solution was prepared by diluting the sample 10 times. Thirty aliquots of the stock solution were then prepared with a volume of $50 \mu \mathrm{L}$ per aliquot.

A mixture of 15 acid standards was prepared at a concentration of $10 \mu \mathrm{g} / \mathrm{mL}$ per acid. The acids included L-proline, L-cystine, L-histidine, L-phenylalanine, L-tyrosine, L-lysine, L-glutamic acid, L-aspartic acid, L-leucine, nonadecanoic acid, hepadecanoic acid, heptanoic acid, nonanoic acid, pentadecanoic acid, and undecanoic acid. Twenty $\mu \mathrm{L}$ of the acid mixture was added to each of the first 10 aliquots of the stock solution, while 24 and $100 \mu \mathrm{L}$ of the acid mixture were added to each of the second 10 aliquots and the third 10 aliquots, respectively. Methanol was then added to each of the 30 aliquots to make the total volume of each aliquot to $200 \mu \mathrm{L}$. This resulted in three sample groups with spiked-in acid standards. The acid concentration in each of the spike-in sample groups is $1.0 \mu \mathrm{g} / \mathrm{mL}, 1.2 \mu \mathrm{g} / \mathrm{mL}$, and $5.0 \mu \mathrm{g} / \mathrm{mL}$, respectively. 


\subsubsection{FTICR-MS and LTQ-MS/MS Analysis}

The direct infusion experiments were performed on a hybrid mass spectrometer, linear trap quadruple - Fourier transform ion cyclotron mass spectrometer (LTQ-FTICR MS or LTQ-FT MS) (Thermo Electron Corporation, Bremen, Germany) equipped with a chip-based nano-electrospray ionization (nESI) ion source (Triversa NanoMate) (Advion Biosciences, Ithaca, NY, USA). The mass spectrometer was operated in positive ion mode. Each metabolite extract was analyzed for 5 minutes with an $\mathrm{m} / \mathrm{z}$ range of 50-1,600. Mass spectra were recorded using FTICR in the profile mode and the resolving power (RP) was set at 200,000@ $\mathrm{m} / \mathrm{z}=400$. The maximum ion accumulation time was set at 1,000 ms. The ion optics was tuned for the sodium adduct of tricaprylin $\left(\left[\mathrm{C}_{27} \mathrm{H}_{50} \mathrm{O}_{6}+\mathrm{Na}^{+}\right]\right)$ at $m / z=493.25$ using the linear ion trap (LIT). The two most important nESI parameters were as follows: the spray voltage $=+1.8 \mathrm{kV}$ and the nitrogen gas pressure $=0.5 \mathrm{psi}$. The MS/MS spectrum of each metabolite ion was acquired with the LTQ-MS. The parameters were set as follows: parent ion $m / z$ isolation window $= \pm 0.5$, spectrum accumulation time $=1 \mathrm{~min}$. The collision-induced dissociation (CID) voltage is a molecule dependent parameter and ranged from 16 to $40 \mathrm{mV}$.

\subsubsection{Data Analysis}

The FT-MS data were processed using the software package MetSign[76]. For metabolite peak quantification, the raw instrument data were first reduced into a peak list using second-order polynomial fitting (SPF) and Gaussian mixture model (GMM). Cross sample alignment was performed using a two-step alignment approach implemented in MetSign, the primary alignment and partial alignment. After peak alignment, a contrast 
based method was employed for normalization[77]. Pairwise two-tail $t$-test was used to study the abundance change of each metabolite between two testing sample groups.

For metabolite identification, each peak detected in the FTICR-MS data was first assigned to metabolite(s) recorded in the public databases (Kyoto Encyclopedia of Genes and Genomes (KEGG, http://www.genome.jp/kegg/), LIPID MAPS (http://www.lipidmaps.org/), and the Human Metabolome Database (HMDB, http://www.hmdb.ca/), by matching the experimentally measured metabolite ion $\mathrm{m} / \mathrm{z}$ value and the profile of the isotopic peaks with the theoretical data of database metabolites. The thresholds for $\mathrm{m} / \mathrm{z}$ variation and isotopic peak profile similarity measured by Pearson's correlation coefficient were set as $\leq 5 \mathrm{ppm}$ and $\geq 0.75$, respectively. To narrow down the metabolite candidates of the initial assignment, the MS/MS spectra were acquired for metabolites detected with significant abundance changes between two testing sample groups. Each experimental MS/MS spectrum was compared to the in silico MS/MS spectra of all candidate metabolites generated by Mass Frontier 7.0 (Thermo Scientific, FL, US). The spectral similarity between the experimental MS/MS spectrum and the in silico MS/MS spectrum of metabolite of interest was evaluated using Pearson's correlation coefficient. The metabolite candidate(s) with the best MS/MS spectral similarity was (were) considered as the metabolite giving rise to the experimental spectrum, while the other candidates were discarded. The authentic standards of the metabolites of interest were then analyzed on LTQ-MS/MS to further confirm the metabolite identification. 
To evaluate the accuracy of both the analytical platform and the data analysis method, the true-positive rate (TPR), the positive predictive value (PPV), and their harmonic mean F1 score were calculated as follows:

$$
\begin{gathered}
T P R=\frac{T P}{T P+F N} \\
P P V=\frac{T P}{T P+F P} \\
F 1=\frac{2 \times T P R \times P P V}{T P R+P P V}
\end{gathered}
$$

where TP (true-positive) is the number of spiked-in acids that were detected as molecules with significant peak area changes between groups of spike-in samples by the statistical analysis, FP (false-positive) is the number of molecules that were not spiked-in acids but detected as molecules with significant peak area changes, and FN (false-negative) is the number of spiked-in acids that were not detected as molecules with significant peak area changes. TPR is called recall, and PPV is called precision and their harmonic mean F1 score can be used as an accuracy of the statistical significant test.

\subsection{Results and Discussion}

\subsubsection{PCB 153 Worsened Steatosis in Mice Fed a High Fat Diet (HFD)}

Oil Red O staining is used in histological visualization of fat cells and neutral fat. Oil Red $\mathrm{O}$ is a dye used for staining of neutral triglycerides and lipids on frozen sections. Haematoxylin and eosin $(\mathrm{H} \& \mathrm{E})$ staining protocol is used frequently in histology to examin thin section of tissue. Haematoxylin stains cell nuclei blue, while eosin stains 
cytoplasm, connective tissue and other extracellular substances pink or red. Histological examination showed that mice fed a control diet did not develop significant steatosis with or without PCB 153 administration (Oil Red O and H\&E stains-Figure 2. A\&B). Mice fed HFD showed minimal steatosis, but PCB 153 co-exposure drastically augmented this effect. Thus, co-administration of PCB 153 to HFD-fed mice clearly worsened hepatic steatosis while PCB 153 had no effect in the control diet fed-mice.

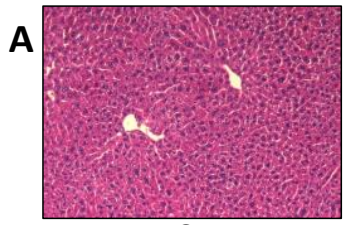

CD

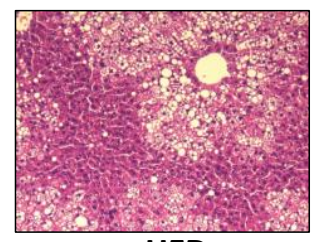

HFD

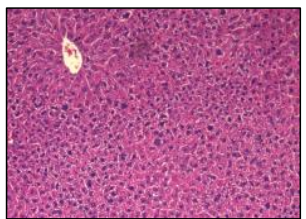

CD+PCB 153

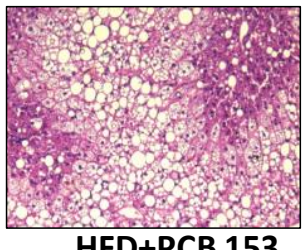

B

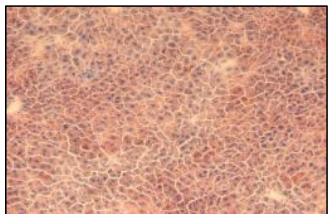

CD

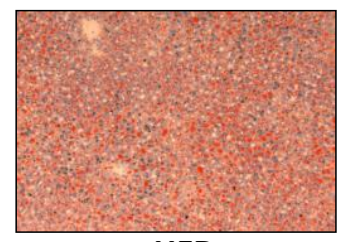

HFD

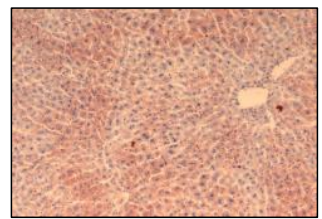

CD+PCB 153

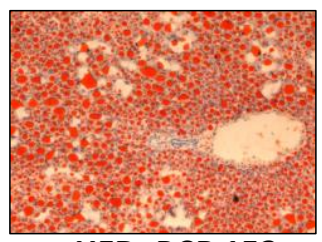

HFD+PCB 153

Figure 2. PCB 153 worsened hepatic steatosis in mice fed a high fat diet. (A) The HFD+PCB 153 mice showed macrovesicular steatosis by H and E staining (B) Oil Red O staining of hepatic sections established the occurrence of micro-vesicular steatosis in the same group of mice.

\subsubsection{Evaluation of Metabolomics Analysis Platform}

The FTICR-MS data of the spiked-in experiment were processed using MetSign[75]. Of the 15 spiked-in acids, the metabolite peaks of 10 acids (L-proline, L-cystine, L-histidine, L-phenylalanine, L-tyrosine, L-lysine, L-glutamic acid, L-aspartic acid, L-leucine, and nonadecanoic acid) were recognized based on the match of $\mathrm{m} / \mathrm{z}$ values and isotopic peak profile. On the basis of the design of the spike-in experiment, all of the 10 detected spiked-in acids are the true-positive metabolites that have different concentrations 
between the two sample groups, while all other metabolites are false-positives if they are detected as the metabolites with significant concentration change between two sample groups. Of the 10 detected acids, L-histidine, L-lysine, and nonadecanoic acid were already present in the liver metabolite extract before the addition of the acid standards. The true concentration ratios of these three acids between samples groups depend on the amount of these acid originally present in the liver.

A pairwise two-tail $t$-test was performed to recognize the metabolite peaks with significant peak area changes between the two testing groups constructed from the three sample groups of the spike-in experiment. Figure 3 displays the relation between the $p$-value threshold of the $t$-test and the recall, the precision and F1, respectively. With the increase in the $p$-value threshold, the TPR increases and it reaches the highest value of 0.80 at a $p$-value of 0.03 . There is a relatively large deviation between the PPV values of these three pairs of testing groups. The best PPV value reaches a value of 0.63 when comparing testing groups $1.0 \mu \mathrm{g} / \mathrm{mL} v s .1 .2 \mu \mathrm{g} / \mathrm{mL}$, while it is only 0.30 for $1.0 \mu \mathrm{g} / \mathrm{mL} v s$. $5.0 \mu \mathrm{g} / \mathrm{mL}$ and $1.2 \mu \mathrm{g} / \mathrm{mL} v s .5 .0 \mu \mathrm{g} / \mathrm{mL}$ at a $p$-value of 0.05 . The $\mathrm{F} 1$ value ranges from 0.43 to 0.70 at a $p$-value of 0.05 . The high value of TPR and moderate value of F1 indicate that the analytical platform and the data analysis method employed in this study are able to detect the metabolites with abundance changes from the biological samples. However, the analytical platform variations also introduce a certain level of false-positive discovery, resulting moderate values of PPV and F1. 

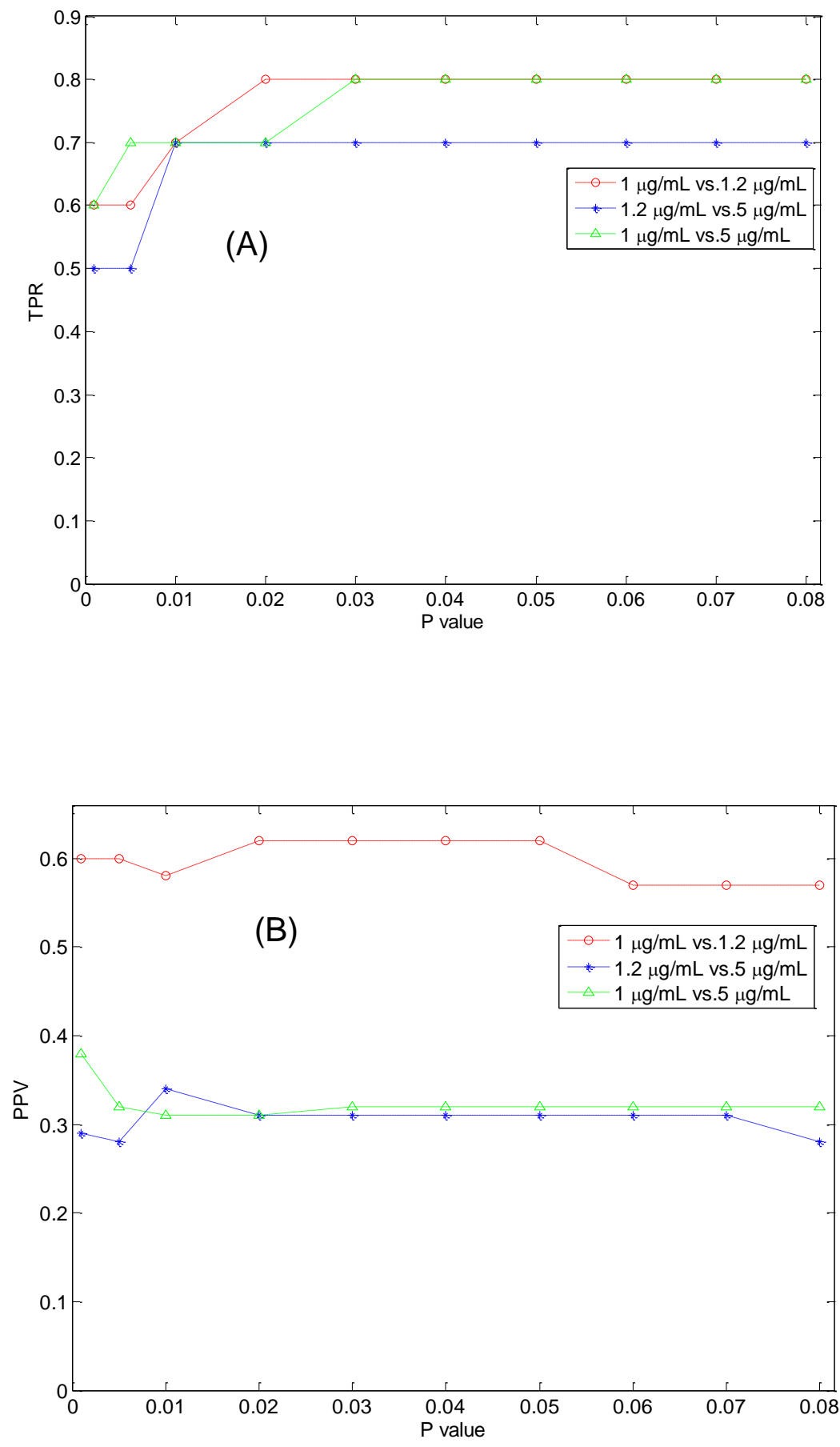


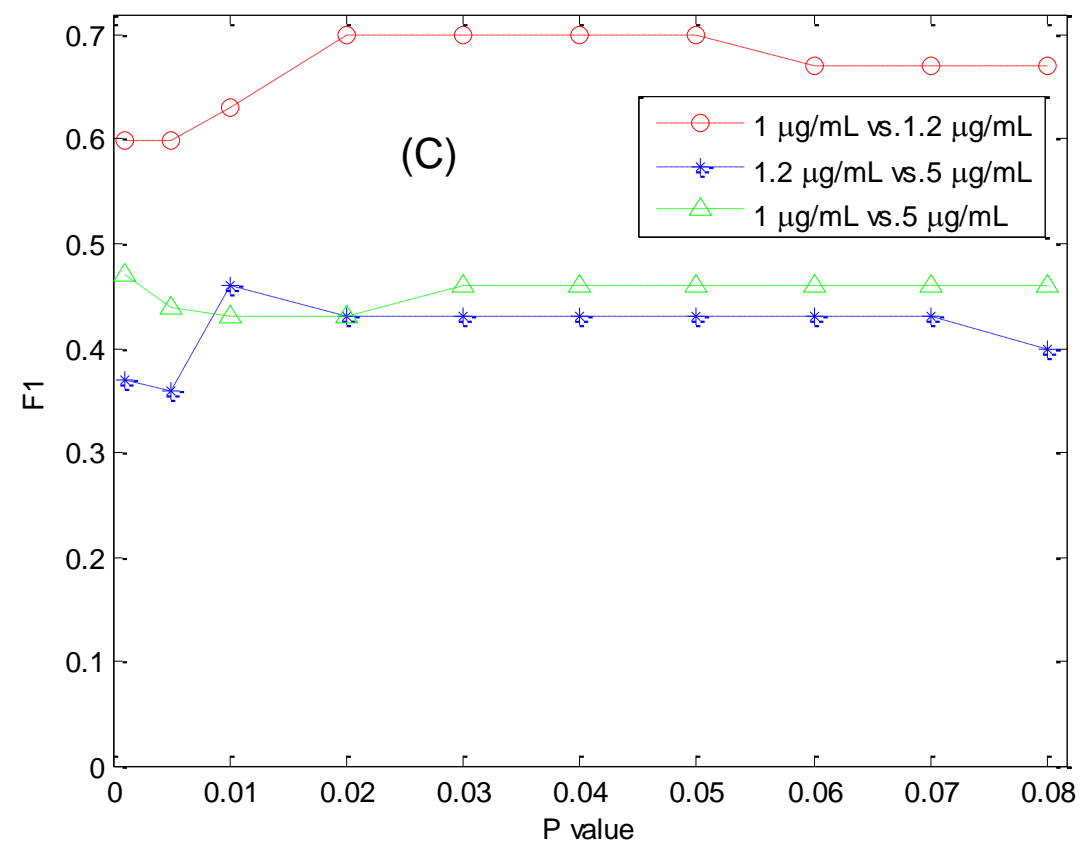

Figure 3. Relationship between the cutoff value of the p-value of t-test and the values of TPR, PPV, and F1 during the analysis of the spiked-in experiments. (A) TPR (B) PPV (C) F1

\subsubsection{Metabolite Identification}

The metabolite putative assignment was accomplished by MetSign[75] software using the FTICR-MS data. About 800 metabolite peaks were putatively assigned to at least one database metabolite with an $\mathrm{m} / \mathrm{z}$ variation window of $\leq 5 \mathrm{ppm}$ and a minimum value of 0.75 as Pearson's correlation coefficient between the theoretical isotopic peak profile and the experimental one. To the metabolite peaks detected with significant abundance changes between two testing sample groups, an experimental MS/MS spectrum was acquired for each of these peaks on LTQ-MS/MS. An in silico MS/MS spectrum was generated for each of the putative metabolite candidates assigned to the metabolite peaks with significant abundance changes. The in silico MS/MS spectra were then matched to 
the experimental MS/MS spectrum of the metabolite ion. The metabolite candidate with the best MS/MS spectral match was considered as the metabolite present in the sample. Figure 4 depicts an example of MS/MS identification. The molecular ion $m / z$ value of the peak measured by FTICR-MS is 156.07731, which was tentatively assigned to the metabolite L-histidine (a metabolite recorded in human metabolite database with database identification number HMDB00177) with an $\mathrm{m} / \mathrm{z}$ value deviation of $0.06 \mathrm{ppm}$ and the isotopic peak profile similarity of 0.9974 . The in silico MS/MS spectrum of this putatively assigned metabolite was obtained using Mass Frontier, and the in silico MS/MS spectrum (Figure 4A) is highly similar to the experimental spectrum (Figure 4B) with a Pearson's correlation coefficient of 1.00. Figure 4 shows the MS/MS spectrum of authentic L-histidine standard. The Pearson's correlation coefficient between the MS/MS spectrum of the authentic L-histidine and the experimental MS/MS spectrum acquired from the biological sample is 0.85 . Such a high spectral similarity significantly increases the identification confidence of L-histidine from the biological samples.

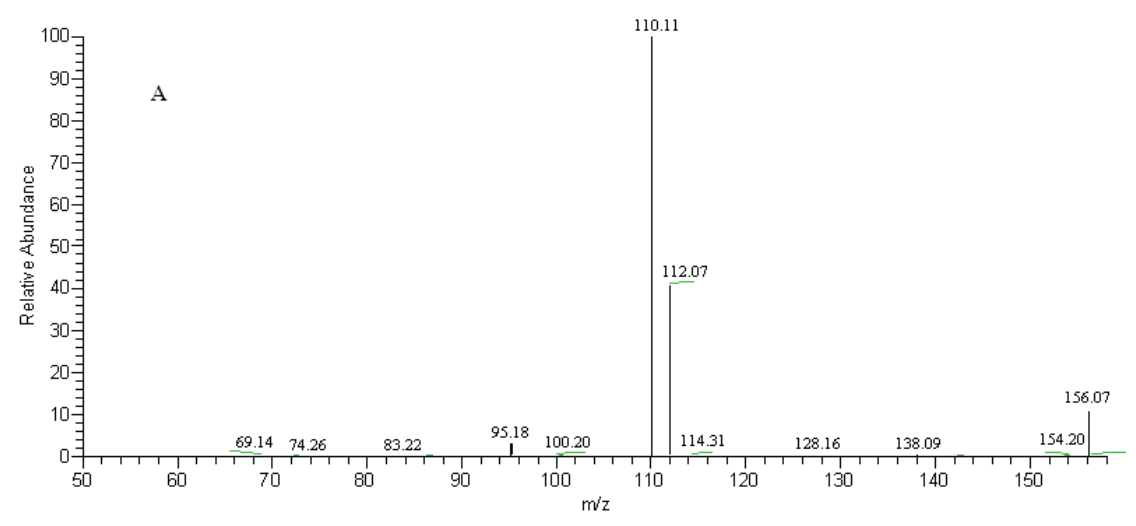



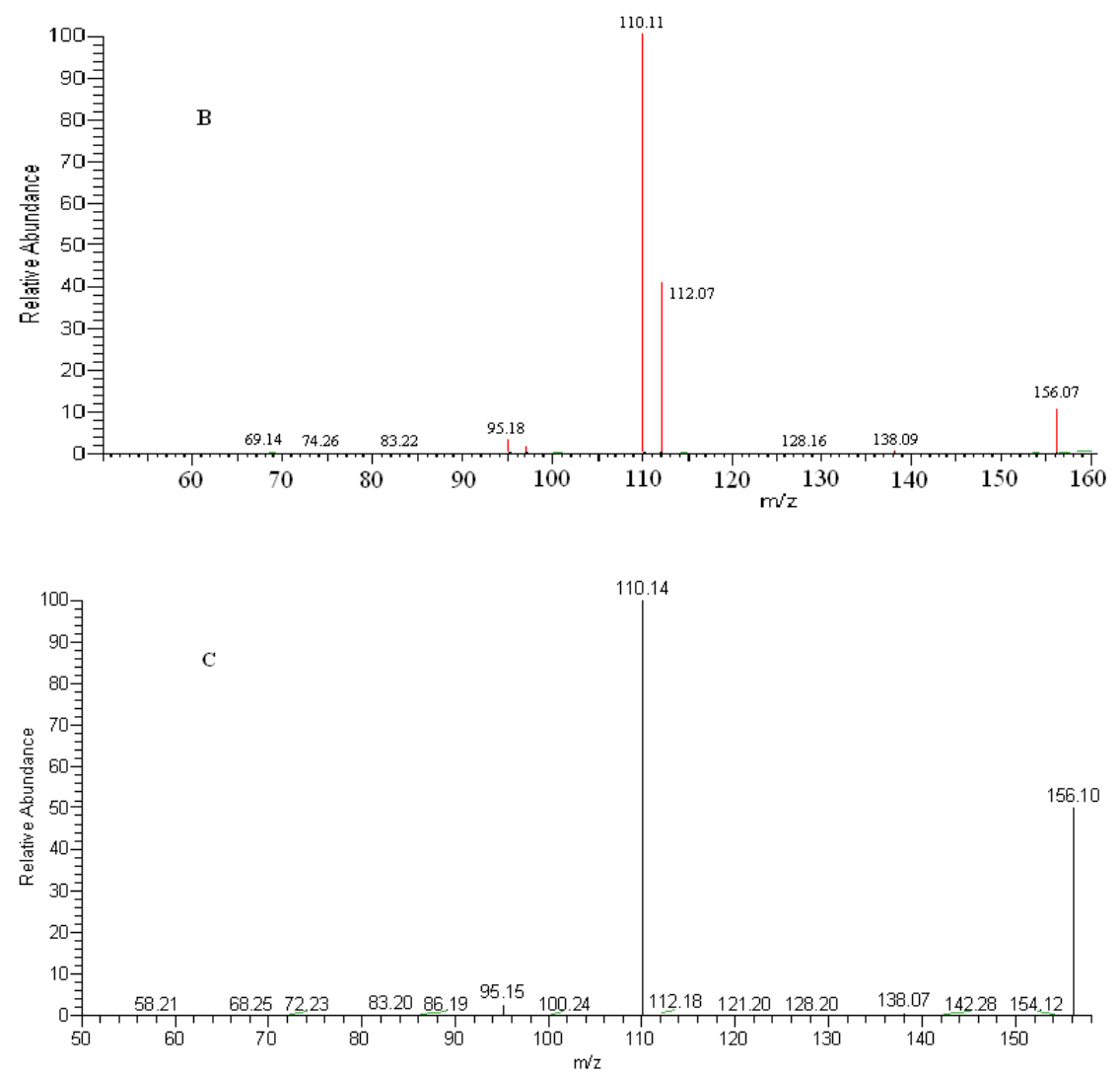

Figure 4. An example of identifying metabolites using MS/MS information. The metabolite ion $m / z$ value was measured using FTICR-MS as 156.0773. (A) is the experiment MS/MS spectrum of the metabolite ion. (B) is the matching result of the metabolite between the experiment MS/MS spectrum and the in silico MS/MS spectrum of the same metabolite ion generated by Mass Frontier 7.0. The matched fragment ions are highlighted in red and the unmatched fragment ions in black. (C) is the MS/MS spectrum of the L-histidine standard.

\subsubsection{Metabolite Quantification}

Four sample groups were generated in this study, including CD group (animals fed a control diet), CD+PCB 153 group (animals fed a control diet with exposure to PCB 153), HFD group (animals fed a high fat diet), and HFD+PCB 153 group (animals fed a high fat diet with exposure to PCB 153). To investigate the metabolite abundance change 
between two sample groups, pairwise two-tail $t$-test was employed with a $p$-value threshold of $\leq 0.05$. Four pairs of sample groups were compared in this study after normalization. Specifically, we compared the metabolite profile difference between CD group and $\mathrm{CD}+\mathrm{PCB} 153$ group, $\mathrm{CD}$ group and $\mathrm{HFD}$ group, $\mathrm{CD}+\mathrm{PCB} 153$ group and HFD+PCB 153 group, and HFD group and HFD+PCB 153 group.

In order to have high metabolite coverage, two samples were collected from each mouse liver: water phase metabolite extract and organic phase metabolite extract. The statistical test was applied to the water phase samples and the organic phase samples, respectively. The test results were then merged for summary. A few metabolites were detected in both the organic phase samples and the water phase samples, but none of them was recognized as a molecule with significant abundance changes between two sample groups. For each of the comparisons, the peak distribution of each metabolite in the samples of the two testing sample groups was generated. Figure 5 depicts a sample peak intensity distribution of metabolites recognized with significant abundance changes between the HFD group and the HFD+PCB 153 group. It can be seen that the abundance of this metabolite (fucose 1-phosphate) is significantly decreased in the HFD+PCB 153 group with a fold-change of 2.65 and a $p$-value of $4.84 \times 10^{-4}$. 


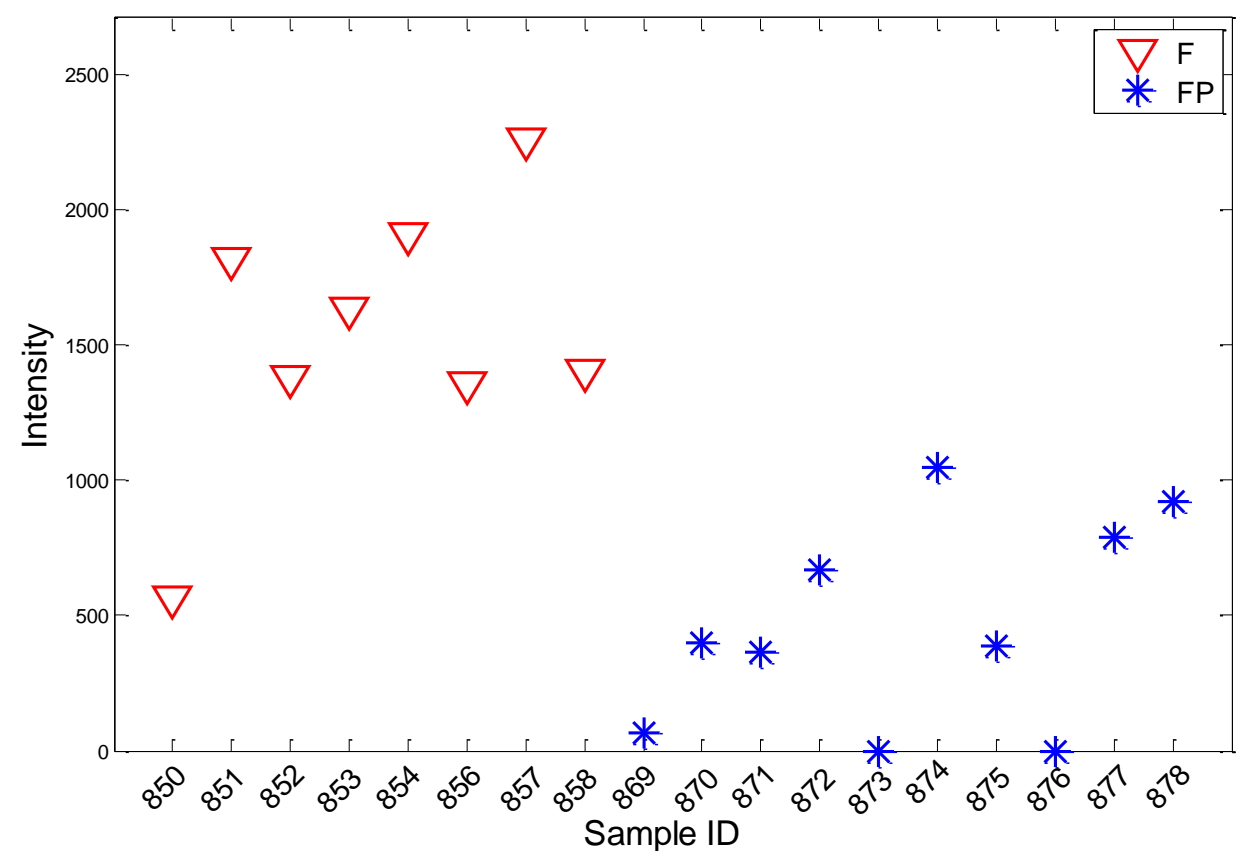

Figure 5. The peak area distribution of metabolite fucose 1-phosphate in the HFD samples (red triangles) and in the HFD+PCB 153 samples (blue stars). The abundance test (pairwise two-tail $t$-test) shows that the regulation of this metabolite in the HFD+PCB 153 group is decreased with a fold change of 2.65 and a $p$-value of $4.84 \times 10^{-4}$.

To measure the metabolite abundance changes between two sample groups, the term fold-change was defined as the ratio of the large abundance value (peak area) of a metabolite in one group divided by the small abundance value of the same metabolite in the other group. The positive sign and negative sign indicate the abundance increase and decrease in the testing group, respectively. No metabolite was detected with significant abundance changes between the $\mathrm{CD}$ group and the $\mathrm{CD}+\mathrm{PCB} 153$ group. This indicates that PCB 153 alone has no significant effect on liver at the metabolite level. However, a total of 14 metabolites were detected with significant abundance changes between the HFD and HFD+PCB 153 groups, as listed in Table 1. Among the metabolites with 
significant changes between the HFD and HFD+PCB 153 groups, erythronic acid (Figure.6) had the largest abundance changes with a 28.8-fold increase in the HFD+PCB 153 group $\left(p=8.35 \times 10^{-5}\right)$. Erythronic acid is formed either by oxidation of D-N-acetyl glucosamine, an alternating unit of hyaluronic acid [78] or by degradation of ascorbic acid (Vitamin C) and glycated proteins[79]. Compared with the changes observed between the CD group and CD+PCB 153 group, the metabolite abundance changes between the HFD and HFD+PCB 153 groups demonstrated the presence of a diet-toxin interaction between PCB 153 and HFD. This interaction may affect some metabolic pathways, and therefore induce the abundance changes of these measured compounds. Thus, PCB 153 alone does not induce NAFLD but it worsens NAFLD caused by a HFD, which agrees with our histological examination (Figure 2).

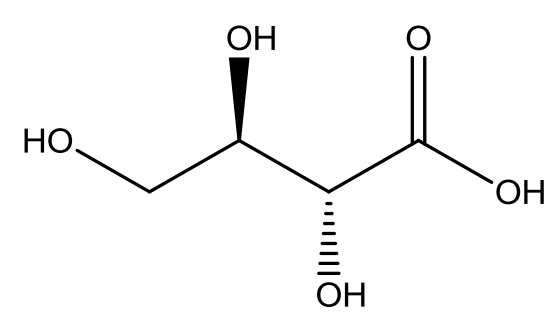

Figure 6. Erythronic Acid Structure 
Table 1. Metabolites detected with significant abundance changes between the HFD group and the HFD+PCB 153 group.

\begin{tabular}{|c|c|c|c|c|c|}
\hline$m / z$ & Name & $\begin{array}{l}\text { Adduct } \\
\text { ion }\end{array}$ & $\begin{array}{l}\text { Metabolite database } \\
\text { IDs }\end{array}$ & $\begin{array}{l}\text { fold } \\
\text { change }\end{array}$ & p_value \\
\hline 156.07731 & L-Histidine & $\mathrm{H}^{+}$ & C00135,HMDB00177 & -1.80 & 4.64E-02 \\
\hline 170.03332 & Creatine & $\mathrm{K}^{+}$ & HMDB00064,C00300 & -1.83 & 4.72E-02 \\
\hline 175.00113 & Erythronic acid & $\mathrm{K}^{+}$ & HMDB00613 & 28.77 & $8.35 \mathrm{E}-05$ \\
\hline 260.05434 & D-Glucosamine6-phosphate & $\mathrm{H}^{+}$ & $\mathrm{C} 00352$ & -2.19 & 4.14E-03 \\
\hline 262.07009 & Fucose 1-phosphate & $\mathrm{NH}_{4}^{+}$ & HMDB01265 & -2.65 & 4.84E-04 \\
\hline 376.05929 & S-(Hydroxymethyl)glutathione & $\mathrm{K}^{+}$ & HMDB04662 & -2.97 & $1.14 \mathrm{E}-02$ \\
\hline 346.0469 & Glutathione & $\mathrm{K}^{+}$ & HMDB00125,C00051 & -5.91 & $2.04 \mathrm{E}-03$ \\
\hline 459.25103 & Stearoylglyceronephosphate & $\mathrm{Na}^{+}$ & $\mathrm{C} 03805$ & -1.67 & $3.14 \mathrm{E}-02$ \\
\hline 266.153 & Isobutylphendienamide & $\mathrm{Na}^{+}$ & C10944 & -2.57 & $1.71 \mathrm{E}-02$ \\
\hline 309.16728 & Fructoselysine & $\mathrm{H}^{+}$ & C16488 & -2.94 & $5.96 \mathrm{E}-03$ \\
\hline 347.1235 & Fructoselysine & $\mathrm{K}^{+}$ & C16488 & -2.08 & $1.70 \mathrm{E}-02$ \\
\hline 283.03409 & Uridine & $\mathrm{K}^{+}$ & HMDB00296,C00299 & -1.80 & 4.99E-02 \\
\hline 559.30352 & Cucurbitacin $\mathrm{P}$ & $\mathrm{K}^{+}$ & LMST01010114 & -2.02 & $1.25 \mathrm{E}-02$ \\
\hline 685.39477 & $\begin{array}{l}\text { 15alpha,25-dihydroxy-16beta,23R:16alpha,24S-diepoxy- } \\
\text { 9beta,19-cyclolanostan-3beta-yl } \\
\text { 2-O-acetyl-alpha-L-arabinopyranoside }\end{array}$ & $\mathrm{Na}^{+}$ & LMST01100006 & 2.05 & $3.68 \mathrm{E}-03$ \\
\hline
\end{tabular}

For the CD+PCB 153 vs. HFD+PCB 153 groups, 50 FTICR-MS peaks were detected with significant abundance changes in water phase samples and 14 peaks in organic phase samples. Combining the water phase and the organic phase results, 24 metabolites were identified by in silico MS/MS spectral matching, although three of them $(\mathrm{m} / \mathrm{z}=$ 369.1178, 203.0535 and 365.1075) do not have unique identification (Table 2). The identified metabolites include glycerolipids, sterol lipids, phospholipids, sphingolipids and other small molecules. It should be noted that the FTICR-MS peaks with $\mathrm{m} / \mathrm{z}=$ 
309.1673 and 347.1235 were both identified as fructoselysine with different adduct ions $\mathrm{H}^{+}$and $\mathrm{K}^{+}$, respectively. The same fold change (-2.43) between the two testing sample groups confirms the accuracy of our FTIRC-MS and data analysis platforms. Another case is the identification of FTICR-MS peaks with $\mathrm{m} / z=156.0773$ and 194.0335. The in silico MS/MS spectral matching identified these two peaks as being generated by the metabolite L-histidine, with adduct ions $\mathrm{H}^{+}$and $\mathrm{K}^{+}$, respectively. The fold changes of these two FTICR-MS peaks between the two testing groups are -1.90 and -1.94 , respectively.

For the CD vs. HFD groups, 57 FTICR-MS peaks were detected having significant abundance different between the CD group and the HFD group in the water phase samples and 17 peaks in the organic phase samples. Combining the results of the water phase and the organic phase samples, 25 metabolites were further confirmed by MS/MS spectral matching. Of the 25 compounds, two do not have a unique identification (Table 3). It should be noted that metabolite PC (o-22:1(13Z)/20:4(8Z,11Z,14Z,17Z)) has a 4.44-fold increase in the HFD group, which agrees with a previous study[80].

Several important limitations in the study design could impact the generalizability of these results. First, differences existed between CD and HFD, not only in macronutrient content and source, but also in micronutrient composition. However, similar, but not identical, vitamin and mineral mixes were given with each diet. These potential confounders could impact metabolite differences observed between diet groups regardless of PCB administration. However, the primary study objective was to determine the effects of PCB administration within a given diet (e.g. HFD vs. HFD+PCB 153, and $\mathrm{CD}$ vs. $\mathrm{CD}+\mathrm{PCB} 153$ ), and these analyses would be unaffected. Thus, the most 
Table 2. Metabolites detected with significant abundance changes between the CD+PCB 153 group and the HFD+PCB 153 group.

\begin{tabular}{|c|c|c|c|c|c|}
\hline$m / z$ & Metabolite Name & $\begin{array}{l}\text { Adduct } \\
\text { ion }\end{array}$ & Metabolite database IDs & $\begin{array}{l}\text { Fold } \\
\text { change }\end{array}$ & p_value \\
\hline 156.0773 & L-histidine & $\mathrm{H}^{+}$ & C00135,HMDB00177 & -1.90 & $1.18 \mathrm{E}-02$ \\
\hline 194.0335 & L-histidine & $\mathrm{K}^{+}$ & C00135,HMDB00177 & -1.95 & $2.77 \mathrm{E}-02$ \\
\hline 227.0439 & $\mathrm{~N}$-acetylglutamine & $\mathrm{K}^{+}$ & HMDB06029 & -2.17 & $2.23 \mathrm{E}-02$ \\
\hline 175.0011 & Erythronic acid & $\mathrm{K}^{+}$ & HMDB00613 & 16.73 & $2.90 \mathrm{E}-04$ \\
\hline 219.0274 & 2S,4S,5R,6-tetrahydroxy-hexanoic acid & $\mathrm{K}^{+}$ & LMFA01050392 & -2.81 & $1.64 \mathrm{E}-03$ \\
\hline 249.0383 & Chorismate & $\mathrm{Na}^{+}$ & HMDB12199 & -2.38 & $3.39 \mathrm{E}-03$ \\
\hline 260.0543 & D-glucosamine6-phosphate & $\mathrm{H}^{+}$ & C00352 & -1.84 & $4.85 \mathrm{E}-02$ \\
\hline 268.1054 & Adenosine & $\mathrm{H}^{+}$ & HMDB00050 & -1.64 & $2.76 \mathrm{E}-02$ \\
\hline 286.2757 & heptadecasphing-4-enine & $\mathrm{H}^{+}$ & LMSP01040002 & 3.29 & $5.64 \mathrm{E}-03$ \\
\hline 347.1235 & Fructoselysine & $\mathrm{K}^{+}$ & C16488 & -2.43 & $3.62 \mathrm{E}-02$ \\
\hline 309.1673 & Fructoselysine & $\mathrm{H}^{+}$ & C16488 & -2.43 & $4.25 \mathrm{E}-02$ \\
\hline 346.0469 & Glutathione & $\mathrm{K}^{+}$ & C00051,HMDB00125 & -19.86 & $9.13 \mathrm{E}-03$ \\
\hline 376.0593 & S-(hydroxymethyl)glutathione & $\mathrm{K}^{+}$ & HMDB04662 & -3.67 & $5.56 \mathrm{E}-04$ \\
\hline 381.0813 & Melibiose & $\mathrm{K}^{+}$ & HMDB00048 & -3.79 & $8.55 \mathrm{E}-04$ \\
\hline 402.0964 & S-lactoylglutathione & $\mathrm{Na}^{+}$ & C03451,HMDB01066 & -3.51 & $9.51 \mathrm{E}-04$ \\
\hline 559.3035 & Cucurbitacin $\mathrm{P}$ & $\mathrm{K}^{+}$ & LMST01010114,C08804 & -1.87 & $1.87 \mathrm{E}-02$ \\
\hline 867.6961 & PC(o-22:1(13Z)/20:4(8Z,11Z,14Z,17Z)) & $\mathrm{NH}_{4}^{+}$ & HMDB13451 & 2.45 & $1.20 \mathrm{E}-03$ \\
\hline 615.4948 & 1,2-di-(9Z-heptadecenoyl)-sn-glycerol & $\mathrm{Na}^{+}$ & LMGL02010021 & -2.37 & $6.24 \mathrm{E}-04$ \\
\hline 685.3948 & $\begin{array}{l}\text { 15alpha,25-dihydroxy-16beta,23R:16alpha,24S-diepoxy-9be } \\
\text { ta,19-cyclolanostan-3beta-yl } \\
\text { 2-O-acetyl-alpha-L-arabinopyranoside }\end{array}$ & $\mathrm{Na}^{+}$ & LMST01100006 & 2.25 & $5.01 \mathrm{E}-04$ \\
\hline 903.7553 & $\begin{array}{l}\text { 1-docosanoyl-2-(15Z-tetracosenoyl)-sn-glycero-3-phosphoet } \\
\text { hanolamine }\end{array}$ & $\mathrm{NH}_{4}^{+}$ & LMGP02010292 & 8.09 & $1.13 \mathrm{E}-03$ \\
\hline 899.7505 & $\begin{array}{l}\text { 1-heptadecanoyl-2-(9Z-heptadecenoyl)-3-octadecanoyl-sn-gl } \\
\text { ycerol }\end{array}$ & $\mathrm{K}^{+}$ & LMGL03010071 & 5.86 & $1.44 \mathrm{E}-05$ \\
\hline \multirow[t]{2}{*}{369.1178} & O-feruloylquinate & $\mathrm{Na}^{+}$ & $\mathrm{C} 02572$ & -3.29 & $2.01 \mathrm{E}-02$ \\
\hline & Aucubin & $\mathrm{Na}^{+}$ & C09771,LMPR0102070006 & & \\
\hline \multirow[t]{2}{*}{203.0535} & D-glucose & $\mathrm{Na}^{+}$ & C00031 & -2.22 & $5.73 \mathrm{E}-03$ \\
\hline & 2S,4S,5R,6-tetrahydroxy-hexanoic acid & $\mathrm{Na}^{+}$ & LMFA01050392 & & \\
\hline \multirow[t]{2}{*}{365.1075} & Melibiose & $\mathrm{Na}^{+}$ & C05402,HMDB00048 & -5.86 & $5.38 \mathrm{E}-05$ \\
\hline & Sucrose & $\mathrm{Na}^{+}$ & C00089 & & \\
\hline
\end{tabular}


Table 3. Metabolites detected with significant abundance changes between the CD group and the HFD group.

\begin{tabular}{|c|c|c|c|c|c|}
\hline$m / z$ & Metabolite Name & $\begin{array}{l}\text { Adduct } \\
\text { ion }\end{array}$ & Metabolite database IDs & $\begin{array}{l}\text { Fold } \\
\text { Change }\end{array}$ & p_value \\
\hline 175.0011 & Erythronic acid & $\mathrm{K}^{+}$ & HMDB00613 & -26.70 & $4.16 \mathrm{E}-02$ \\
\hline 219.0274 & 2S,4S,5R,6-tetrahydroxy-hexanoic acid & $\mathrm{K}^{+}$ & LMFA01050392 & -1.72 & $1.21 \mathrm{E}-02$ \\
\hline 251.054 & 2-Hydroxy-3-carboxy-6-oxo-7-methylocta-2,4-dienoate & $\mathrm{Na}^{+}$ & $\mathrm{C} 06581$ & -2.25 & $4.58 \mathrm{E}-03$ \\
\hline 262.0701 & Fucose 1-phosphate & $\mathrm{NH}_{4}^{+}$ & HMDB01265 & 1.93 & $5.55 \mathrm{E}-03$ \\
\hline 286.2757 & heptadecasphing-4-enine & $\mathrm{H}^{+}$ & LMSP01040002 & 3.92 & $1.74 \mathrm{E}-03$ \\
\hline 355.0671 & 2-Caffeoylisocitrate & $\mathrm{H}^{+}$ & $\mathrm{C} 02927$ & -1.92 & 4.54E-02 \\
\hline 364.1238 & Lactosamine & $\mathrm{Na}^{+}$ & HMDB06591 & 1.77 & $9.17 \mathrm{E}-03$ \\
\hline 365.0966 & Streptidine6-phosphate & $\mathrm{Na}^{+}$ & $\mathrm{C} 01121$ & -1.80 & $6.33 \mathrm{E}-03$ \\
\hline 365.102 & Hinokitiolglucoside & $\mathrm{K}^{+}$ & $\mathrm{C} 15451$ & -3.93 & $3.16 \mathrm{E}-04$ \\
\hline 380.0977 & Lactosamine & $\mathrm{K}^{+}$ & HMDB06591 & 1.36 & $4.94 \mathrm{E}-02$ \\
\hline 381.069 & Streptidine6-phosphate & $\mathrm{K}^{+}$ & $\mathrm{C} 01121$ & -2.36 & $1.41 \mathrm{E}-03$ \\
\hline 384.1218 & Acutumidine & $\mathrm{H}^{+}$ & $\mathrm{C} 10565$ & -1.87 & $1.26 \mathrm{E}-02$ \\
\hline 390.3365 & $\begin{array}{l}\text { N-(5-hydroxy-pentyl)-5Z,8Z,11Z,14Z-eicosatetraenoyl } \\
\text { amine }\end{array}$ & $\mathrm{H}^{+}$ & LMFA08020020 & -4.94 & $3.06 \mathrm{E}-02$ \\
\hline 399.0858 & Elephantopin & $\mathrm{K}^{+}$ & LMPR0103090004,C09403 & -2.55 & $5.92 \mathrm{E}-03$ \\
\hline 459.251 & Stearoylglyceronephosphate & $\mathrm{Na}^{+}$ & $\mathrm{C} 03805$ & 2.10 & $1.33 \mathrm{E}-03$ \\
\hline 497.3475 & 25-Hydroxy-24-epi-brassinolide & $\mathrm{H}^{+}$ & $\mathrm{C} 11050$ & 2.05 & $4.42 \mathrm{E}-02$ \\
\hline 519.3296 & 25-Hydroxy-24-epi-brassinolide & $\mathrm{Na}^{+}$ & $\mathrm{C} 11050$ & 1.96 & $7.21 \mathrm{E}-03$ \\
\hline 527.1377 & 3-Fucosyllactose & $\mathrm{K}^{+}$ & HMDB02094 & -2.53 & $1.15 \mathrm{E}-02$ \\
\hline 535.3036 & 25-Hydroxy-24-epi-brassinolide & $\mathrm{K}^{+}$ & $\mathrm{C} 11050$ & 1.64 & $2.11 \mathrm{E}-02$ \\
\hline 615.4948 & 1,2-di-(9Z-heptadecenoyl)-sn-glycerol & $\mathrm{Na}^{+}$ & LMGL02010021 & -3.66 & $3.02 \mathrm{E}-02$ \\
\hline 663.0984 & Isoorientin 3'-O-glucuronide & $\mathrm{K}^{+}$ & LMPK12110311 & -5.87 & $6.50 \mathrm{E}-04$ \\
\hline 867.6961 & PC(o-22:1(13Z)/20:4(8Z,11Z,14Z,17Z)) & $\mathrm{NH}_{4}^{+}$ & HMDB13451 & 4.44 & $5.25 \mathrm{E}-05$ \\
\hline 879.6859 & $\begin{array}{l}\text { 1-heptadecanoyl-2,3-di-(9Z,12Z-heptadecadienoyl)-sn-glyce } \\
\text { rol }\end{array}$ & $\mathrm{K}^{+}$ & LMGL03010067 & 28.39 & $1.34 \mathrm{E}-05$ \\
\hline \multirow[t]{2}{*}{203.0535} & 2S,4S,5R,6-tetrahydroxy-hexanoic acid & $\mathrm{Na}^{+}$ & LMFA01050392 & -1.47 & $2.23 \mathrm{E}-02$ \\
\hline & D-Glucose & $\mathrm{Na}^{+}$ & $\mathrm{C} 00031$ & & \\
\hline \multirow[t]{2}{*}{365.1075} & Melibiose & $\mathrm{Na}^{+}$ & C05402,HMDB00048 & -3.18 & $1.49 \mathrm{E}-04$ \\
\hline & Sucrose & $\mathrm{Na}^{+}$ & C00089 & & \\
\hline
\end{tabular}


important conclusion from our work is that macronutrient-toxicant interactions are critical determinants of PCB 153's effects on hepatic metabolites. Additional consideration must also be given to the PCB dosing protocol. PCBs were manufactured as mixtures, and multiple highly chlorinated PCB congeners have simultaneously bio-accumulated in humans primarily by ingestion[68]. This study investigated only a single congener (PCB 153), which is the single most abundant PCB in humans[1].

Because a metabolomics dose response curve has never been performed for any PCB, for this initial study a relatively high cumulative dose $(200 \mathrm{mg} / \mathrm{kg}$, administered to mice i.p., over 12 weeks) was selected based on a previously published National Toxicology Program Protocol (NTP TR 530, $210 \mathrm{mg} / \mathrm{kg}$ cumulative dose, administered to rats by gavage over 14-weeks. The NTP protocol produced lipid-adjusted serum PCB levels approximately 10-fold higher than the most highly exposed subject from the Anniston, Alabama human cohort of highly-exposed residents living near a former PCB production facility[81]. Limitations regarding our PCB dosing protocol must be acknowledged and future studies investigating PCB mixtures at lower doses and more physiologic routes of administration (e.g. gavage) have been planned. However, the most important finding of this study is that even at a relatively high dose of PCB 153, no effects on hepatic metabolites were observed in the absence of high fat feeding. Therefore, the data suggest that the PCB-HFD interaction could be more important than the cumulative PCB dose.

\subsubsection{Ingenuity Pathway Analysis (IPA)}

As demonstrated by the spike-in experimental data, the analytical platform and the data analysis method employed in this study can introduce a certain level of technical variations. Such variations can cause both false-positive and false-negative discoveries. 
On the other hand, the quantitative analysis was performed on the abundance of each individual metabolite. The inter-relationships between the various metabolites were not considered however, and thus, IPA was employed. IPA correlates specifically targeted metabolites with potential metabolic pathways for data analysis that helps researchers to model, analyze, and understand complex biological and chemical systems at the core of life science research[82]. Therefore, it is necessary to incorporate the analytical discovery with the metabolite pathway analysis to further filter and/or enrich the analytical discovery.

All 14 metabolites recognized with significant abundance changes between the HFD group and the HFD+PCB 153 group were subjected to IPA for network analysis. Six metabolites were mapped into the IPA database. The most probable metabolite network reported by IPA contained 5 metabolites including creatine, glucosamine-6-phosphate, glutathione, L-histidine and uridine with a score of 14 (Figure 7). The IPA analysis resulted in that the top hepatotoxicity function is glutathione (GSH) depletion in liver and the top canonical pathway is glutamate metabolism. GSH, a tripeptide composed of glutamic acid, cysteine and glycine, is the major intracellular anti-oxidant in the liver, and its physiological function is to prevent damage to cellular components that may be caused by xenobiotic metabolites, reactive oxygen species (ROS) and free radicals[83]. In the liver, glutamate is the terminus for release of ammonia from amino acids, and the intrahepatic concentration of glutamate modulates the rate of ammonia detoxification into urea[83,84].

GSH (entry 7 of Table 1) had a 5.91-fold decrease in the HFD+PCB 153 group ( $p$-value of $2.03 \times 10^{-3}$ ) and its conjugate, $S$-(hydroxymethyl)glutathione (entry 6) had a 2.97 -fold 
decrease ( $p$-value of $1.14 \times 10^{-2}$ ). Hepatic mitochondrial GSH depletion has been associated with the progression of alcoholic liver disease[85] and blood GSH depletion with obesity and diabetes[86]. Lower hepatic GSH content has also been reported in non-alcoholic steatosis[87]. Furthermore, Swenberg et al. reported the formation of oxidative DNA lesions in PCB 126-exposed rats which was likely due to ROS generation[88]. Therefore, the GSH depletion noted in the steatotic livers of mice treated with HFD+PCB 153 implies increased oxidative stress leading to extensive utilization of GSH which eventually decreased liver GSH stores. Further evidence for oxidative stress can also be accounted for by the increased levels of erythronic acid, the degradation product of vitamin $\mathrm{C}$, another crucial anti-oxidant. The mechanism by which PCB 153 induces oxidative stress needs further investigation. PCB 153 is very poorly metabolized[89] and therefore conjugation of its metabolites is very unlikely to deplete glutathione. 


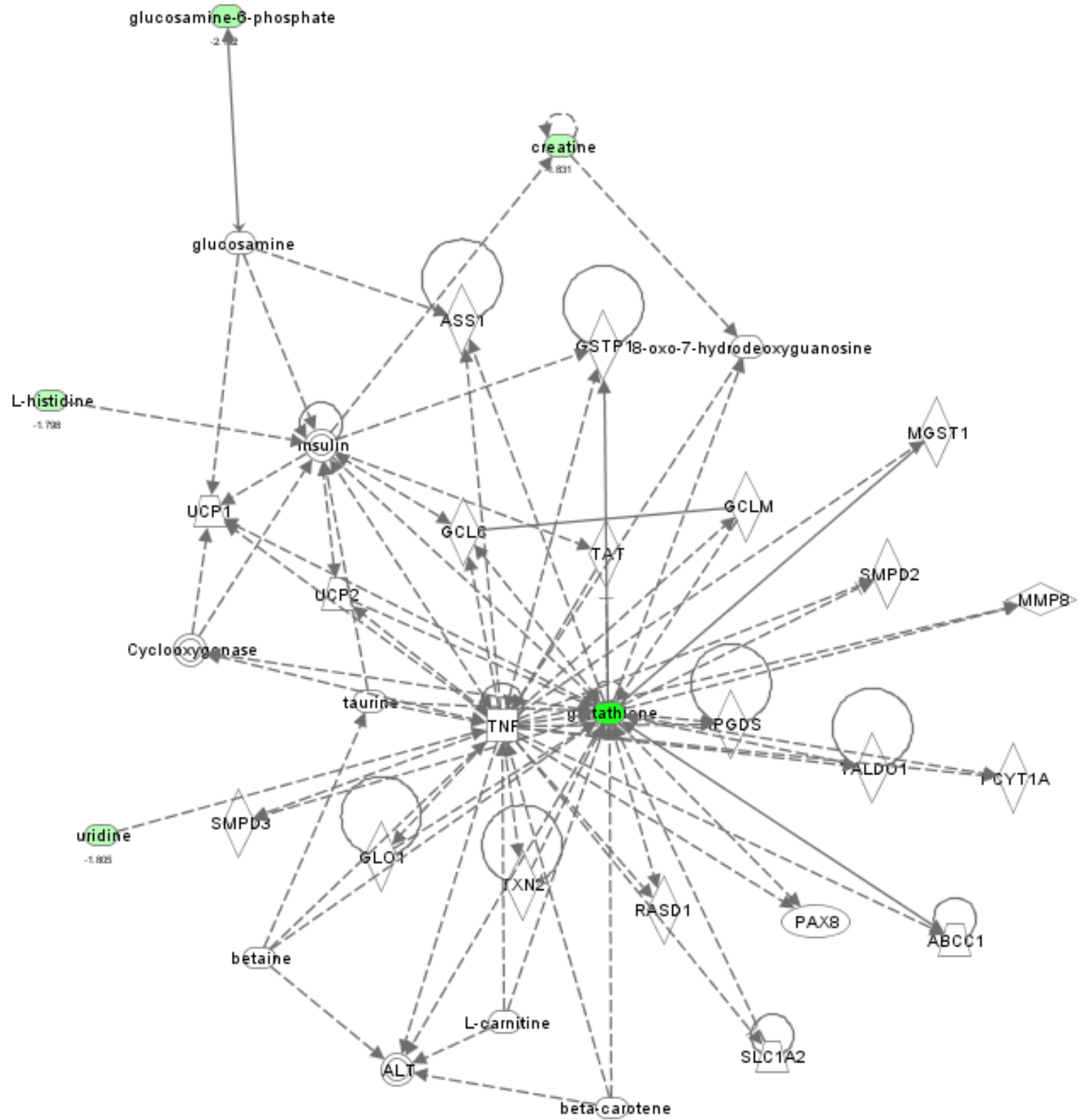

Figure 7. IPA proof-of-knowledge characterization of the metabolic networks in mouse liver affected by PCB 153.The dashed line indicates indirect interactions between metabolites while the solid line means a direct metabolite interaction. Filled circles represent metabolites discovered in this work and the color of each filled circle represents the direction and magnitude of fold-changes. Green color means down-regulated, red color means up-regulated. 
Unlike PCB 126, which is a potent Ah-receptor agonist strongly inducing CYP1A activity, PCB 153 is reported to have weak "Phenobarbital" activity[90] suggesting it will be a relatively poor inducer of CYP2B and thus is expected to have relatively poor ability to induce monooxygenase activity which could increase the levels of reactive compounds that reduce glutathione levels. However, it is possible that the depletion could be due to glutathione consumption by increased lipid peroxides which should have been generated within the steatotic livers of the HFD+PCB 153 group. It must also be noted that our results are consistent with the findings of Twaroski et al., as we observed no effects of PCB 153 on glutathione in the presence of a normal rodent chow diet[91]. PCB 153-mediated glutathione depletion occurring only in the presence of HFD is not only innovative, but also probably the most important finding in this study.

Regardless of its mechanism, the reduction in glutathione (entry 7 of Table 1) would diminish its availability for transformation into $S$-(hydroxymethyl)glutathione (entry 6), and should trigger the up-regulation of glutathione biosynthesis from glutamate, glycine, and cysteine, its component amino acids. Glutamate is made from the TCA cycle intermediate 2-oxoglutarate and ammonia, which could account for the decrease in measured D-glucosamine 6-phosphate (entry 4), either from its use as an ammonia source or from lowered production due to ammonia depletion. Histidine (entry 1) can also be rationalized as an ammonia source by decarboxylation and oxidation or as a source of both ammonia and glutamate via the pathway initiated by the action of histidine ammonia-lyase. The synthesis of uridine (entry 12) likely falls as ammonia is shunted into glutamate production. The degradation of creatine (entry 2) gives rise to glycine through the action of creatinase and sarcosine dehydrogenase. Interestingly, reduced 
serum creatine levels in humans and mouse models has recently been reported in a metabolomic profiling studies of steatosis[92] and NAFLD progression[93]. Such a simple accounting of glutathione consumption upon exposure of the liver to PCB 153, therefore, provides good rationalization of the observed reduction in metabolite levels (Table 1), centered on the glutamate metabolism.

IPA mapped 7 of the 24 metabolites recognized with significant abundance changes between the $\mathrm{CD}+\mathrm{PCB} 153$ group and $\mathrm{HFD}+\mathrm{PCB} 153$ group. The most probable metabolite network reported by IPA contained 6 metabolites including adenosine, L-histidine, glutathione, D-glucose, melibiose and S-lactoylglutathione with a score of 16. The top hepatotoxicity function is glutathione depletion in liver and the top canonical pathway is also glutamate metabolism. D-glucose was detected with a 2.22-fold decrease in the HFD+PCB 153 group, indicating a significant decrease of D-glucose in the liver due to the exposure to PCB 153. D-glucose has been used as a biomarker for diagnosis of liver cancer[35]. GSH was detected with a 19.9-fold decrease in the HFD+PCB 153 group, and this provides further evidence in the importance of HFD in PCB 153-mediated GSH depletion.

Seven of the 25 metabolites with significant abundance changes between the CD group and HFD group were mapped to the IPA database. The most probable metabolite network reported by IPA has a score of 11 and contains 5 metabolites including stearoylglyceronephosphate, fucose 1-phosphate, melibiose, streptidine 6-phosphate, and 2-Hydroxy-3-carboxy-6-oxo-7-methylocta-2,4-dienoate. 


\subsection{Conclusions}

Polychlorinated biphenyls (PCBs) are persistent environmental pollutants. The hepatic effects of PCB 153 in mice were investigated via a direct infusion nano-electrospray ionization linear trap quadrupole - Fourier transform ion cyclotron resonance mass spectrometry (DI-nESI-LTQ-FTICR MS). No difference was observed in the metabolite profile of mice a fed control diet with or without PCB 153 exposure. However, when mice fed a high fat diet were compared with mice fed a control diet, 15 metabolites were reduced and 10 metabolites were increased. Compared to the $\mathrm{CD}+\mathrm{PCB} 153$ group, 18 metabolites were reduced while 6 metabolites were increased in the HFD+PCB 153 group. Compared with the HFD group, 12 metabolites were reduced including glutathione and creatine and 2 metabolites were increased, most notably erythronic acid, in the HFD+PCB 153 group. These data indicate that PCB 153 had no observable effects on metabolites when administered to mice fed $\mathrm{CD}$, which is consistent with the absence of histopathology. In contrast, when administered with HFD, PCB 153 produced significant metabolic changes (vs. HFD, or CD+PCB 153), which are consistent with worsened obesity/NAFLD pathology. Thus, the metabolic effects of PCB 153 were heavily dependent on macronutrient interactions with HFD. Antioxidant depletion is likely to be an important consequence of this interaction, as this mechanism has previously been implicated in obesity/NAFLD. 


\section{CHAPTER 3}

\section{CHRONIC ALCOHOL EXPOSURE DISTURBS LIPID HOMEOSTASIS AT THE ADIPOSE-LIVER AXIS: ANALYSIS OF TRIACYLGLYCEROLS USING HIGH-RESOLUTION MASS SPECTROMETRY IN COMBINATION WITH IN VIVO METABOLITE DEUTERIUM LABELING}

\subsection{Introduction}

It has been understood that dietary fats are digested in the intestinal epithelial cells, and then converted to triacylglycerols (TGs). TGs are assembled with apolipoproteins to form chylomicrons which are transported into the blood stream via the lymph system[94]. TGs are also synthesized by the liver where they are packaged as very low-density lipoproteins (VLDL) and secreted into the blood[94]. Upon arrival in the adipose and muscle tissues, lipoprotein lipase cleaves TG into free fatty acids and glycerol. Fatty acids are taken up by these tissues, and are used as energy sources via oxidation in muscles, or re-assembled into TGs to store excess energy in the white adipose tissue (WAT)[95]. Glycerol is transported to liver or kidneys where it is converted into dihydroxyacetone phosphate by glycerol kinase and glycerol-3-phosphate dehydrogenase. WAT plays an important role in regulation of whole body energy homeostasis. WAT stores excess energy in the form of TG under positive energy balance condition, and releases fatty acids for energy generation under negative energy balance condition[95]. 
However, excess fatty acid release from the WAT may cause fatty acid overflux into the liver, leading to development of fatty liver[96]. Fatty liver is frequently associated with both alcohol abuse (alcoholic fatty liver, AFL) and obesity (nonalcoholic fatty liver, NAFL). Although previous studies have demonstrated similarities and differences in the pathogenesis of fatty liver between alcohol abuse and obesity, increased fatty acid uptake has been suggested to be a common mechanism for AFL and NAFL[97,98]. While hepatocytes isolated from both alcohol-fed and obese rats showed an increased fatty acid uptake[99], further investigation is needed to provide direct evidence that fatty acids released from the WAT are indeed deposited in the liver.

Clinical studies have demonstrated that lower fat mass (lipodystrophy) was associated with higher liver fat in alcoholics[100,101]. Animal models of AFL also showed that reduction of WAT mass was associated with an increased fatty acid uptake by hepatocytes[102-104]. Our study demonstrated that alcohol exposure to mice caused more hepatic accumulation of TGs which were labeled before alcohol exposure[105]. We also found that alcohol exposure stimulated adipose lipolysis and fatty acid release from WAT[105,106]. These data suggest that alcohol exposure may cause an excess reverse fatty acid transport, thereby inducing fatty liver. Therefore, the animal model of AFL could be an ideal model to identify the importance of WAT in maintaining lipid homeostasis at the WAT-liver axis. Further determination of triacylglycerol homeostasis at the WAT-liver axis could reveal the direct link between WAT and liver, an organ-organ interaction mechanism, in the development of fatty liver.

The objective of this work was to use high-resolution mass spectrometry in combination with metabolite deuterium labeling to test our hypothesis that alcohol exposure disturbs 
lipid homeostasis at the WAT-liver axis towards triacylglycerol epitomic deposition in the liver. In order to differentiate the liver lipids synthesized using fatty acids from other sources from that synthesized using the fatty acids transported back from adipose tissue, a two-stage feeding experiment was performed, where all mice were first fed with deuterated water $\left({ }^{2} \mathrm{H}_{2} \mathrm{O}\right)$ to ensure that the lipids stored in adipose tissue are deuterium labeled (stage one). The mice were then randomly grouped into two cohorts, the control cohort and the test cohort. Mice in the test cohort were fed an alcohol-containing liquid diet while mice in the control cohort were pair-fed an isocaloric maltose dextrin control liquid diet. The mice in both the control and test cohorts were then sacrificed at different times (stage two). Metabolite extracts from mouse liver, epididymal white adipose tissue (eWAT) and subcutaneous white adipose tissue (sWAT) were analyzed using linear trap quadrupole-Fourier transform ion cyclotron resonance mass spectrometer (LTQ-FTICR MS) via direct infusion electrospray ionization-mass spectrometry.

\subsection{Experimental}

\subsubsection{Animals and Treatments}

Male C57BL/6N mice were obtained from Harlan (Indianapolis, IN, USA). All the mice were treated according to the experimental procedures approved by the University of Louisville Animal Care and Use Committee. To label lipids in adipose tissues, an approach using ${ }^{2} \mathrm{H}_{2} \mathrm{O}$ as the metabolic tracer was followed[107]. Mice at two months old were given an initial priming dose of $99.8 \%{ }^{2} \mathrm{H}_{2} \mathrm{O}$ via an intraperitoneal injection to achieve $2.5 \%$ of body water enrichment, followed by administration of $5 \%{ }^{2} \mathrm{H}_{2} \mathrm{O}$ in the drinking water for five weeks (stage one, time point 0 week). The mice were then 
randomly grouped into two cohorts, the control cohort and the test cohort, for a 4-week of alcohol exposure (stage two). The test cohort was fed a modified Lieber-DeCarli alcohol liquid diet which contained $1,000 \mathrm{kcal} / \mathrm{L}$ calories, $34 \%$ from alcohol, $18 \%$ from protein, $34 \%$ from fat, and $14 \%$ from carbohydrate. The control cohort was fed a modified Lieber-DeCarli control liquid diet which also contained 1,000 kcal/L calories with replacement of the alcohol calories by isocaloric maltose dextrin. The alcohol-fed mice were free access to the alcohol diet, while the pair-fed mice were given the control diet in the same amount consumed by alcohol-fed mice in the previous day. The liquid diet feeding was conducted for 2 (time point of two weeks) or 4 (time point of four weeks) weeks. At the end of each feeding time point, mice were anesthetized, and liver, eWAT and sWAT tissues were collected from each mouse for measuring lipid components labeled by deuterium. There were 5, 5, 3 and 5, 7, 5 mice at time point $0,2,4$ weeks for the control cohort and the test cohort, respectively.

\subsubsection{Tissue Sample Preparation}

Liver, eWAT and sWAT samples were weighed, homogenized for 2 min and stored at $-80{ }^{\circ} \mathrm{C}$ until use. To extract metabolites from the homogenized tissue, $100 \mu \mathrm{L}$ of homogenized tissue sample, $20 \mu \mathrm{L}$ of butylatedhydroxytoluene (BHT) mixture (50 mg BHT into $1 \mathrm{~mL}$ methanol) and $2.0 \mathrm{~mL}$ chloroform-methanol $(v / v=2: 1)$ were mixed and vortexed for 2 min followed by adding $420 \mu \mathrm{L}$ of water and vortexing for $2 \mathrm{~min}$. The mixture was then centrifuged at room temperature at 2,000 rpm for $8 \mathrm{~min} .400 \mu \mathrm{L}$ of the organic phase (bottom) was aspirated into another glass tube and dried using a nitrogen evaporator. The dried sample was then dissolved into $200 \mu \mathrm{L}$ of chloroform-methanol $(v / v=2: 1)$. 


\subsubsection{FT-MS and LTQ-MS/MS Analysis}

The direct infusion experiments were performed on a hybrid mass spectrometer, the so-called linear trap quadrupole - Fourier transform ion cyclotron resonance mass spectrometer (LTQ-FTICR MS or LTQ-FT MS) (Thermo Electron Corporation, Bremen, Germany) equipped with a chip-based nano-electrospray ionization (nESI) ion source (TriversaNanoMate) (Advion Biosciences, Ithaca, NY, USA). The mass spectrometer was operated in the positive ion mode. Each metabolite extract was measured for 5 min covering the $\mathrm{m} / \mathrm{z}=100-1,600$ range. The mass spectra were recorded using the FTICR in profile mode and the resolving power (RP) was set at 400,000@ m/z=400. The maximum ion accumulation time was set at 1,000 ms. The ion optics was tuned for the sodium adduct of tricaprylin $\left(\left[\mathrm{C}_{27} \mathrm{H}_{50} \mathrm{O}_{6}+\mathrm{Na}^{+}\right]\right)$at $\mathrm{m} / \mathrm{z}=493.25$ using the linear ion trap (LIT). The two most important nESI parameters were as follows: the spray voltage = $+1.8 \mathrm{kV}$ and the nitrogen gas pressure $=0.5 \mathrm{psi}$. The MS/MS spectrum of each metabolite ion was acquired on the LTQ. The parameters were set as follows: precursor ion $\mathrm{m} / \mathrm{z}$ isolation window $= \pm 0.3$, spectrum accumulation time $=1 \mathrm{~min}$. The normalized collision energy (NCE) is a molecule dependent parameter and ranged from 16 to $40 \%$.

\subsubsection{Metabolite Quantification}

The experimental data were processed using software package MetSign[108]. After peak alignment, a contrast based method was employed for normalization[76,77]. Both the Fisher's exact test and the pairwise two-tail t-test were used to study the concentration change of each metabolite between the two physiological conditions. The parameters used during the analysis are as follows: precursor ion $\mathrm{m} / \mathrm{z}$ accuracy $\leq 5 \mathrm{ppm}$ and the 
$\mathrm{q}$-value for false discovery rate $(\mathrm{FDR}) \leq 0.2[109]$. Temporal analysis as performed to study the correlation between time course trajectories measured by Pearson's correlation coefficient and the distance measured by Fisher's combined probability test[110,111].

\subsubsection{Metabolite Identification}

Metabolite identification was achieved in two sequential steps, database search and MS/MS characterization. Such a metabolite identification process meets the requirement of Level 2 metabolite identification, i.e., putatively annotated metabolites[112]. The metabolite database search was accomplished by the MetSign software using the FTICR-MS data. Each of the measured metabolite ion $\mathrm{m} / \mathrm{z}$ value and isotopic peak profile were compared to the corresponding theoretical information of metabolites recorded in the MetSign database, which was composed of all metabolites recorded in the Kyoto Encyclopedia of Genes and Genomes, LIPID MAPS, and the Human Metabolome Database, resulting in 43,245 records. Possible positive-mode adduct ions include $\mathrm{H}+$, $\mathrm{Na}^{+}, \mathrm{K}^{+}$, and $\mathrm{NH}_{4}^{+}$.

To narrow down the metabolite candidates generated by database searching, the MS/MS spectra of metabolite peaks with significant concentration changes between two sample cohorts were acquired on LTQ-MS/MS. Each experimental MS/MS spectrum was compared to the in silico MS/MS spectra of all metabolite candidates using Mass Frontier 6.0 (Thermo Scientific, FL, USA). The spectral similarity between the experimental MS/MS spectrum and the in silico MS/MS spectrum of the metabolite of interest was evaluated using Pearson's correlation coefficient. The metabolite candidate(s) with the best MS/MS spectrum match was (were) considered as the metabolite giving rise to the experimental spectrum. 


\subsubsection{Measurements of Liver Steatosis and Routine Parameters}

Neutral lipids in the liver were detected by Oil red O staining. Liver cryostat sections were cut at $7 \mu \mathrm{M}$, fixed with $10 \%$ formalin for $5 \mathrm{~min}$, and stained with Oil red $\mathrm{O}$ in 2-propynal solution for $10 \mathrm{~min}$. Plasma alanine aminotransferase (ALT) activity and triglyceride and cholesterol concentrations were determined using Infinity Reagents (Thermo Scientific, Middletown, VA). Plasma free fatty acids (FFA) were quantified using a FFA Quantification Kit (BioVision, San Francisco, CA). Statistical differences were analyzed by one-way ANOVA followed by Bonferroni post hoc comparison. The data are presented as mean $\pm \mathrm{SD}$ and $\mathrm{p}$ values less than 0.05 were considered as significant.

\subsection{Results}

\subsubsection{Metabolite Identification}

The metabolite initial assignment via database search was achieved using high-resolution FTICR-MS data. Figure 8 is a sample of putative identification of triacylglycerol TG(16:0/18:2/20:4)[iso6]. The $\mathrm{m} / \mathrm{z}$ value of this metabolite was measured as 904.7407 by FTICR-MS. By searching the 43,245 database metabolites, this metabolite ion and its isotopic peak profile match the corresponding theoretical information of metabolites HMDB05391, HMDB10508, and HMDB13423 with adducts of $\mathrm{Na}^{+}, \mathrm{H}^{+}$and $\mathrm{K}^{+}$, respectively. The number of deuterium atoms incorporated in these three metabolite candidates is 3,5 , and 6 , respectively. Therefore, the $\mathrm{m} / \mathrm{z}$ values of the corresponding 

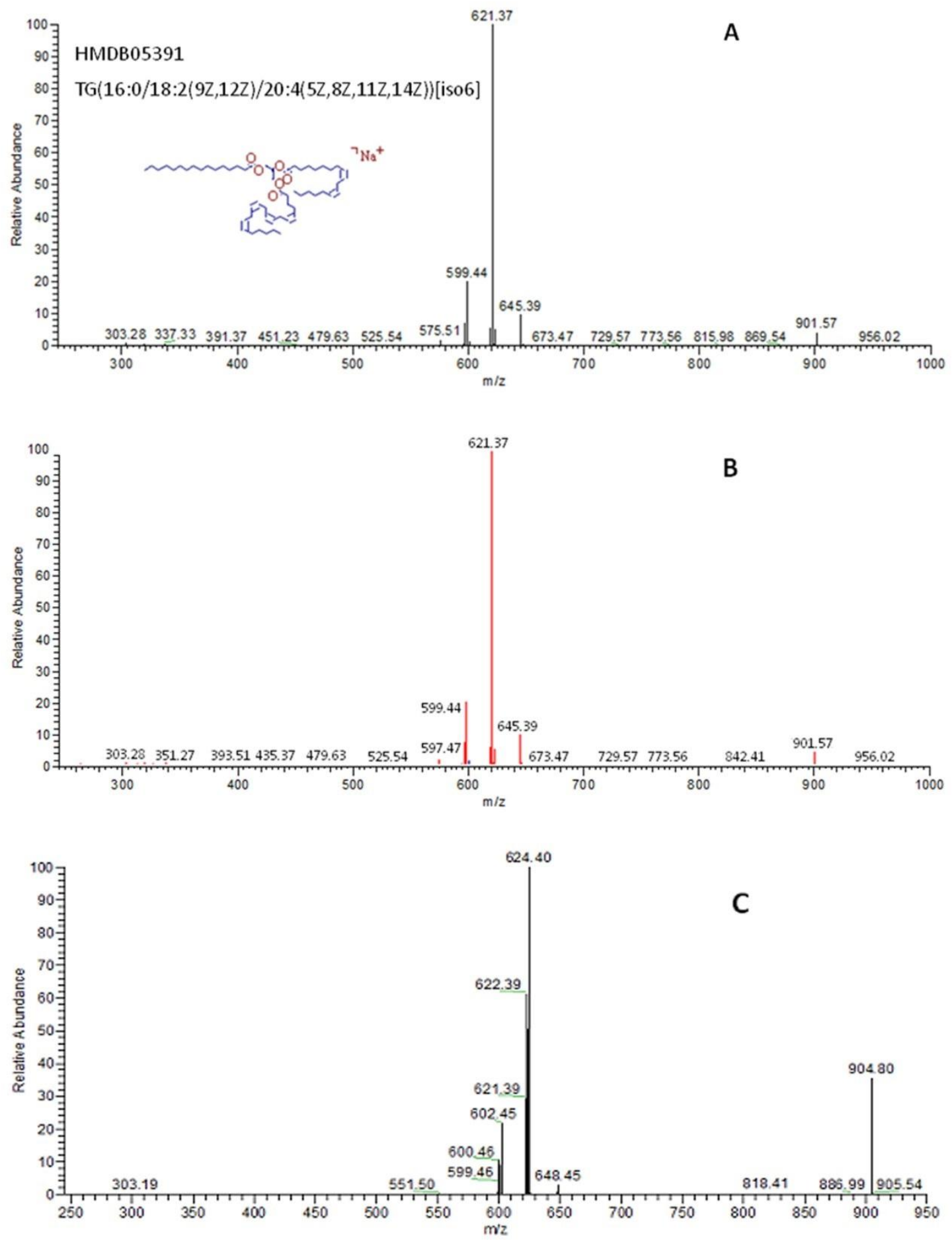

Figure 8. An example of identifying a deuterium incorporated metabolite using MS/MS information. The metabolite ion $m / z$ value was measured on FTICR-MS as 904.74066. (A) is 
the experimental MS/MS spectrum of non-deuterated metabolite. (B) is the matching result of the non-deuterated metabolite between the experiment MS/MS spectrum and the theoretical MS/MS spectrum generated by Mass Frontier. The matched fragment ions are highlighted in red and the not matched ions in black. (C) is the MS/MS spectrum of deuterium incorporated metabolite.

non-deuterium incorporated metabolites of these three metabolite candidates should be 901.7407, 899.7407 and 898.7407, respectively. In order to confirm the initial assignment, LTQ-MS/MS experiments were performed to acquire MS/MS spectra for each of these metabolite ions. Figure 8A is the LTQ-MS/MS spectrum of the metabolite ion with a measured $\mathrm{m} / \mathrm{z}=901.57$. The molecular structures of the candidate metabolites HMDB05391, HMDB10508, and HMDB13423 were then uploaded into Mass Frontier with corresponding adducts $\mathrm{Na}+\mathrm{H}+$ and $\mathrm{K}+$, respectively, to generate in silico MS/MS spectra for each of these three candidates. Each of the in silico spectra was then matched to the experimental LTQ-MS/MS spectrum. The in silico MS/MS spectrum of metabolite HMDB05391+Na+ has the best match with a Pearson's correlation coefficient of 0.9981 and therefore, this metabolite was considered as the metabolite present in the sample (Figure 8B).

It should be pointed out that Mass Frontier software can only predict the $\mathrm{m} / \mathrm{z}$ values of fragment ions, but not the fragment ion abundance. It then matches the $\mathrm{m} / \mathrm{z}$ values of the predicted fragment ions to the $\mathrm{m} / \mathrm{z}$ values of experiment mass spectrum. Therefore, a high value of Pearson's correlation coefficient only refers to the matching quality of fragment ion $\mathrm{m} / \mathrm{z}$ values between an in silico MS/MS and an experiment MS/MS spectrum. 
The incorporated deuterium atoms in a metabolite do not significantly affect the metabolite fragmentation during MS/MS analysis, resulting in similar MS/MS spectra between the deuterium incorporated metabolite and the corresponding non-deuterium incorporated metabolite. The only difference between the MS/MS spectra is $\mathrm{m} / \mathrm{z}$ values of the fragment ions that carry deuterium atoms. The difference in the $\mathrm{m} / \mathrm{z}$ values between the corresponding fragment ions in the two spectra may range from zero to the mass of all incorporated deuterium atoms. Figure $8 \mathrm{C}$ is the LTQ-MS/MS spectrum of a deuterium incorporated version of metabolite HMDB05391 with a measured metabolite ion $\mathrm{m} / \mathrm{z}=904.80$. The corresponding fragment ions between the deuterium incorporated fragment ions and the non-deuterium incorporated fragment ions are (904.80, 901.57), (887.99, 883.62), (648.45, 645.39), (624.40, 621.37), (622.39, 619.44), (602.45, 599.44) and $(600.46,597.47)$. The number of incorporated deuterium atoms in each fragment ion is $3,3,3,3,3,3$ and 3 , respectively. The spectral similarity between the spectrum of a deuterium incorporated metabolite (Figure 8C) and the spectrum of the corresponding non-deuterium incorporated metabolite (Figure 8A) was evaluated using Pearson's correlation coefficient, after recognizing the pairs of fragment ions between the spectrum of a deuterium incorporated metabolite and the spectrum of a non-deuterium incorporated metabolite. The Pearson's correlation coefficient between the top 10 abundant fragment ions in the two spectra displayed in Figures $8 \mathrm{~A}$ and $8 \mathrm{C}$ is 0.8744 , showing the high similarity between these two spectra. 


\subsubsection{Statistical Significance Tests}

Figure 9 depicts peak area distribution of triacylglycerol TG(16:0/18:2/20:4)[iso6] at time two weeks among the samples of the test and control cohorts. It can be seen that this molecule is significantly increased with a 2.8 -fold change in the test cohort compared to its level in the control cohort. The fold change was defined as the ratio of the average peak area of a metabolite measured in the test cohort divided by the average peak area of the same metabolite in the control cohort. The p-value of the pairwise two-tail t-test is $1.4 \times 10^{-5}$.

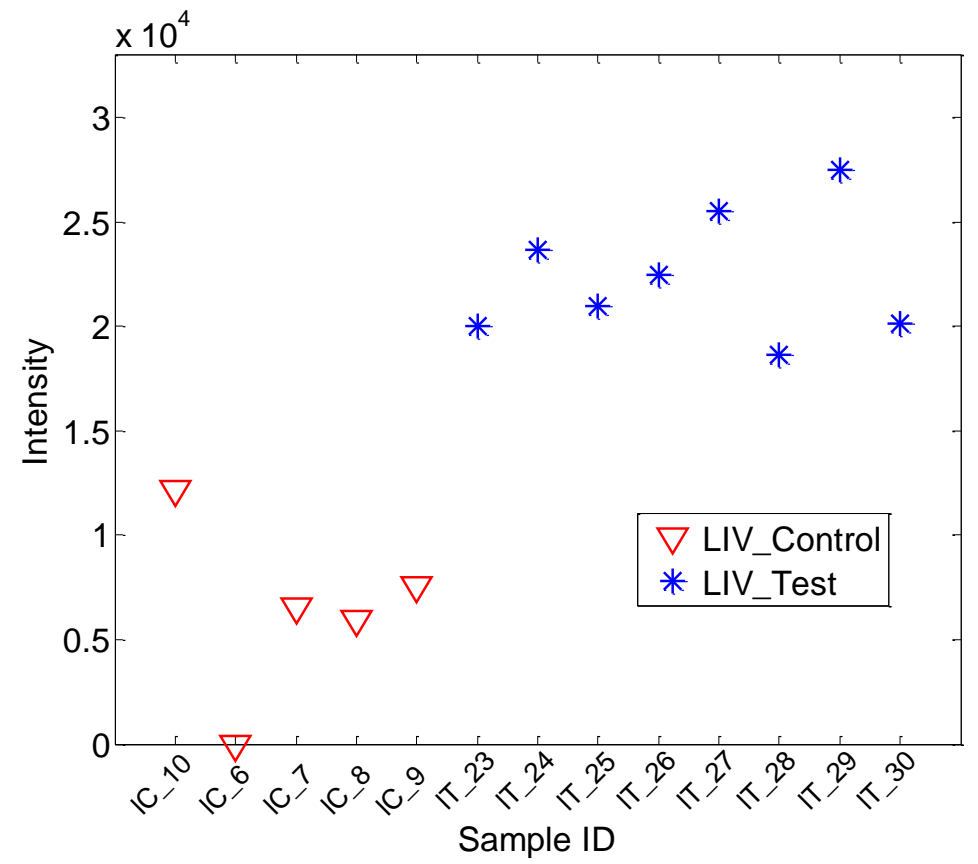

Figure 9. Sample concentration changes of metabolite in two different physiological conditions.The abundance test (pair-wise two-tail t-test) shows that the concentration of this metabolite in the test cohort is increased with a fold change of 2.8 and a p-value of $1.4 \times 10^{-5}$.

This metabolite was further identified as TG(16:0/18:2/20:4)[iso6] by MS/MS analysis. 
Table 4 lists all of the metabolites identified with significant concentration changes in liver between the control cohort and the test cohort at two and four weeks. All of these metabolites were identified as deuterium incorporated TGs even though all the 43,245 metabolites were searched for the metabolite identification. Secondly, the deuterium incorporated TGs, 13 at 2 weeks and 10 at 4 weeks, were all increased in the test cohort with a 1.7 to 6.3 -fold change. Tables 5 list all of the metabolites identified with significant concentration changes between the control cohort and the test cohort at two and four weeks in the eWAT and sWAT. These metabolites are all deuterium labeled and identified as TGs. The number of deuterated TGs was more in the eWAT (10 TGs) compared to the sWAT (4 TGs). All these deuteraed TGs were reduced by alcohol exposure at either two weeks or four weeks with a fold-change ranged from 0.19 to 0.77 .

Table 4. List of triacylglycerols in liver identified with significant concentration changes between the control cohort and the test cohort at two and four weeks.

\begin{tabular}{|c|c|c|c|c|c|c|}
\hline $\begin{array}{l}\text { Time } \\
\text { (Week) }\end{array}$ & $m / z$ & $\mathrm{p}$-value & $\begin{array}{l}\text { Fold change } \\
(\mathrm{T} / \mathrm{C})^{\mathrm{a}}\end{array}$ & Metabolite common name & $\begin{array}{l}\text { Adduct } \\
\text { ion }\end{array}$ & No. ${ }^{2} \mathrm{H}$ \\
\hline 2 & 878.7338 & $7.8 \times 10^{-3}$ & 2.1 & TG(16:1/18:2/20:4)[iso6] & $\mathrm{H}^{+}$ & 1 \\
\hline 2 & 902.7352 & $1.0 \times 10^{-4}$ & 8.1 & TG(16:0/18:2/20:4)[iso6] & $\mathrm{Na}^{+}$ & 1 \\
\hline 2 & 904.7407 & $1.4 \times 10^{-5}$ & 2.8 & TG(16:0/18:2/20:4)[iso6] & $\mathrm{Na}^{+}$ & 3 \\
\hline \multirow[t]{2}{*}{2} & 907.7698 & $2.8 \times 10^{-3}$ & 2.8 & TG(16:0/18:0/20:4)[iso6] & $\mathrm{Na}^{+}$ & 4 \\
\hline & & & & TG(16:0/20:4/20:4)[iso3] & $\mathrm{H}^{+}$ & 4 \\
\hline \multirow[t]{2}{*}{2} & 926.7360 & $6.9 \times 10^{-3}$ & 4.2 & TG(16:0/20:4/20:4)[iso3] & $\mathrm{Na}^{+}$ & 1 \\
\hline & & & & TG(18:3/18:2/22:6)[iso6] & $\mathrm{H}^{+}$ & 1 \\
\hline 2 & 927.7376 & $2.3 \times 10^{-3}$ & 1.9 & TG(16:0/20:4/20:4)[iso3] & $\mathrm{Na}^{+}$ & 2 \\
\hline 2 & 928.7407 & $5.7 \times 10^{-3}$ & 1.9 & TG(16:0/20:4/20:4)[iso3] & $\mathrm{Na}^{+}$ & 3 \\
\hline \multirow[t]{2}{*}{2} & 929.7534 & $1.6 \times 10^{-2}$ & 1.7 & TG(16:0/20:4/20:4)[iso3] & $\mathrm{Na}^{+}$ & 4 \\
\hline & & & & TG(18:3/18:2/22:6)[iso6] & $\mathrm{H}^{+}$ & 4 \\
\hline 2 & 930.7666 & $3.1 \times 10^{-2}$ & 3.1 & TG(18:3/18:3/20:0)[iso3] & $\mathrm{Na}^{+}$ & 1 \\
\hline
\end{tabular}




\begin{tabular}{|c|c|c|c|c|c|c|}
\hline 2 & 952.7521 & $8.6 \times 10^{-3}$ & 6.3 & TG(18:1/20:4/20:4)[iso3] & $\mathrm{Na}^{+}$ & 1 \\
\hline & & & & TG(18:3/20:4/22:6)[iso6] & $\mathrm{H}^{+}$ & 3 \\
\hline 2 & 954.7666 & $9.1 \times 10^{-3}$ & 3.0 & TG(20:4/18:1/22:6)[iso6] & $\mathrm{H}^{+}$ & 1 \\
\hline 2 & 903.7797 & $7.2 \times 10^{-3}$ & $\mathrm{na}^{\mathrm{b}}$ & TG(16:0/18:0/18:0)[iso3] & $\mathrm{K}^{+}$ & 2 \\
\hline \multirow[t]{2}{*}{2} & 905.7533 & $4.8 \times 10^{-3}$ & $\mathrm{na}^{\mathrm{b}}$ & TG(16:0/18:2/20:4)[iso6] & $\mathrm{Na}^{+}$ & 4 \\
\hline & & & & TG(16:0/20:4/20:4)[iso3] & $\mathrm{H}^{+}$ & 2 \\
\hline 4 & 902.7352 & $2.1 \times 10^{-3}$ & 3.8 & TG(16:0/18:2/20:4)[iso6] & $\mathrm{Na}^{+}$ & 1 \\
\hline 4 & 904.7407 & $4.4 \times 10^{-2}$ & 1.7 & TG(16:0/18:2/20:4)[iso6] & $\mathrm{Na}^{+}$ & 3 \\
\hline \multirow[t]{2}{*}{4} & 926.7360 & $1.8 \times 10^{-3}$ & 2.7 & TG(16:0/20:4/20:4)[iso3] & $\mathrm{Na}^{+}$ & 1 \\
\hline & & & & TG(18:3/18:2/22:6)[iso6] & $\mathrm{H}^{+}$ & 1 \\
\hline 4 & 927.7376 & $3.8 \times 10^{-3}$ & 2.5 & TG(16:0/20:4/20:4)[iso3] & $\mathrm{Na}^{+}$ & 2 \\
\hline 4 & 928.7407 & $3.6 \times 10^{-2}$ & 1.7 & TG(16:0/20:4/20:4)[iso3] & $\mathrm{Na}^{+}$ & 3 \\
\hline \multirow[t]{2}{*}{4} & 929.7534 & $2.2 \times 10^{-2}$ & 1.9 & TG(16:0/20:4/20:4)[iso3] & $\mathrm{Na}^{+}$ & 4 \\
\hline & & & & TG(18:3/18:2/22:6)[iso6] & $\mathrm{H}^{+}$ & 4 \\
\hline 4 & 930.7666 & $3.2 \times 10^{-2}$ & 2.8 & TG(18:3/18:3/20:0)[iso3] & $\mathrm{Na}^{+}$ & 1 \\
\hline \multirow[t]{2}{*}{4} & 952.7521 & $2.8 \times 10^{-2}$ & 3.6 & TG(18:1/20:4/20:4)[iso3] & $\mathrm{Na}^{+}$ & 1 \\
\hline & & & & TG(18:3/20:4/22:6)[iso6] & $\mathrm{H}^{+}$ & 3 \\
\hline 4 & 953.7545 & $2.5 \times 10^{-2}$ & 2.7 & TG(18:3/20:4/22:6)[iso6] & $\mathrm{H}^{+}$ & 4 \\
\hline 4 & 954.7666 & $5.3 \times 10^{-3}$ & 3.3 & TG(20:4/18:1/22:6)[iso6] & $\mathrm{H}^{+}$ & 1 \\
\hline
\end{tabular}

a. Fold-change is the ratio of average peak area of a metabolite in the test cohort $(\mathrm{T})$ to that in the control cohort $(\mathrm{C})$.

b. na refers to a metabolite that was detected only in the test cohort. Therefore, the values of fold change for these metabolites are not available.

\subsubsection{Temporal Analysis}

Even though the statistical significance tests support the hypothesis of reverse fatty acid transport, it is still necessary to investigate the trajectory of each metabolite in the time course. Figure 10 shows three sample time course trajectories in liver, eWAT and sWAT, respectively. Figure 10A displays the time course trajectory of triacylglycerol 
TG(16:0/18:2/20:4)[iso6] with one ${ }^{2} \mathrm{H}$ label and one $\mathrm{Na}+$ as adduct ion in liver samples. While this TG molecule was not significantly increased in control cohort, it was significantly increased in test cohort at 2 weeks and a further elevation was found at 4 weeks. Figures 10B and 10C show the time course trajectories of TG(16:0/16:1/16:1)[iso3] with one ${ }^{2} \mathrm{H}$ label and an adduct ion of $\mathrm{Na}+$ in eWAT samples, TG(16:0/16:0/18:1)[iso3] with one ${ }^{2} \mathrm{H}$ label and an adduct ion of $\mathrm{Na}+$ in sWAT samples, respectively. In contrast to the increase in the liver of test cohort, a decrease in deuterium-labeled TGs was observed in both the eWAT and sWAT of test cohorts. The deuterium-labeled TG(16:0/16:1/16:1)[iso3] in eWAT significantly declined at 2 weeks and a further decrease was found at 4 weeks. While deuterium-labeled TG(16:0/16:0/18:1)[iso3] in sWAT significantly declined at 2 weeks, no further decrease was found at 4 weeks. Time course changes for other deuterium-labeled TGs in liver and WAT were listed in Table 4 and Table 5, respectively.

Figure 11 shows that the time course trajectory of triacylglycerol TG(16:0/18:2/20:4)[iso6] without ${ }^{2} \mathrm{H}$ label in liver samples of control cohort and test cohort. This TG molecule represents hepatic TGs which are synthesized from the dietary fats or from de novo lipogenesis, because it did not incorporate any deuterium. In the liver of control cohort, this TG molecule did not change at 2 weeks but increased at 4 weeks. Surprisingly, the test cohort showed a remarkable increase at 2 weeks compared to time 0 , and a further increase at 4 weeks. The abundance of this TG molecule in the test cohort was significantly higher than that in the control cohort at both 2 and 4 weeks. 

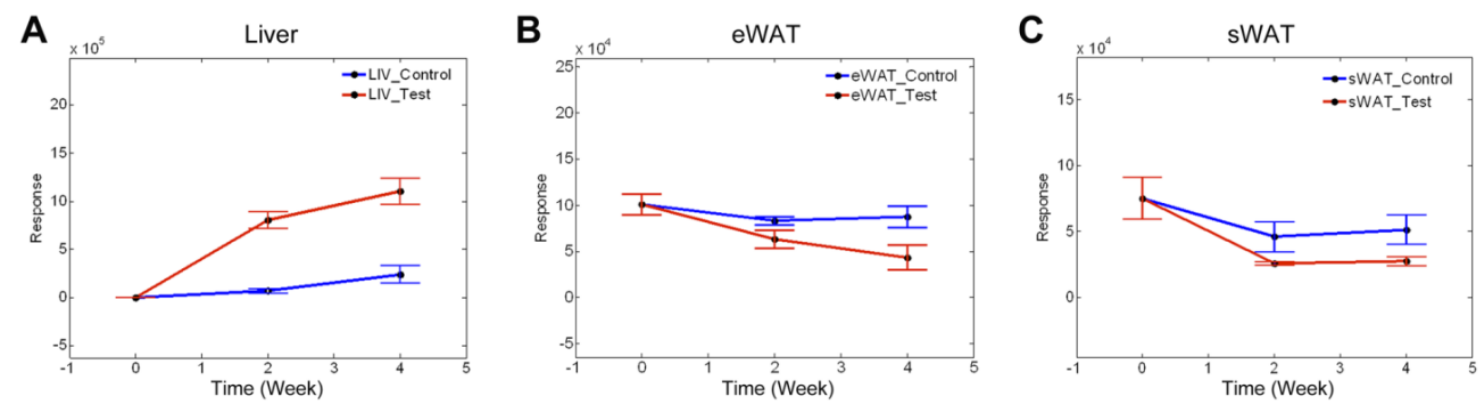

Figure 10. Sample time course trajectories of deuterium labeled triacylglycerols detected in liver, eWAT and sWAT samples.(A) TG(16:0/18:2/20:4)[iso6] with one ${ }^{2} \mathrm{H}$ label and one $\mathrm{Na}^{+}$as adduct ion in liver samples. (B) TG(16:0/16:1/16:1)[iso3] with one ${ }^{2} \mathrm{H}$ label and an adduct ion of $\mathrm{Na}^{+}$eWAT samples. (C) TG(16:0/16:0/18:1)[iso3] with one ${ }^{2} \mathrm{H}$ label and an adduct ion of $\mathrm{Na}^{+}$in sWAT samples

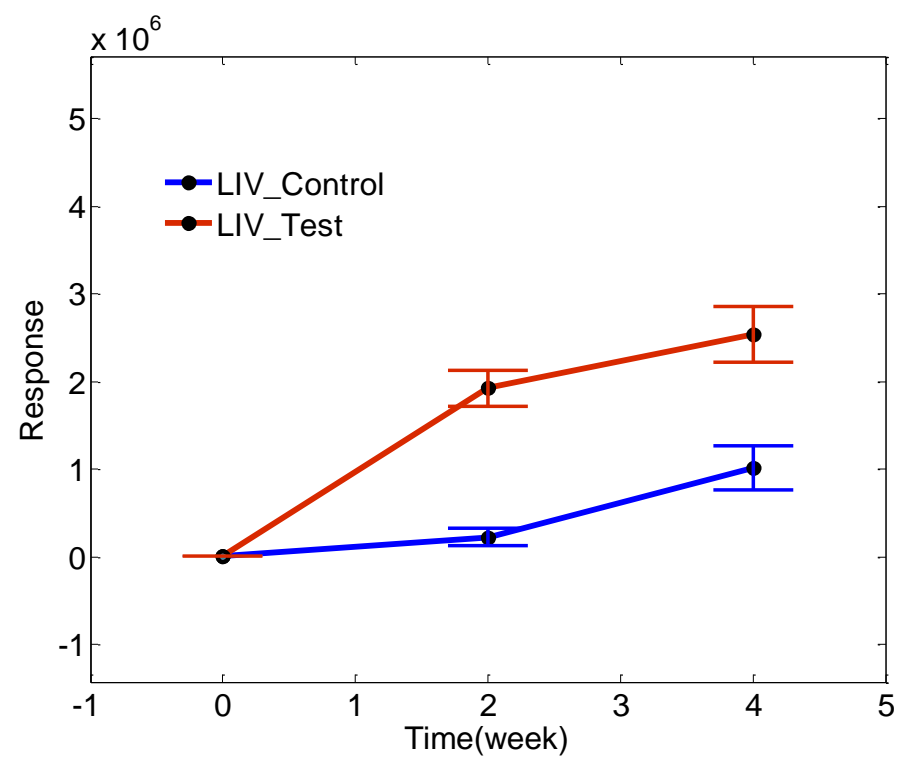

Figure 11. Time course trajectory of triacylglycerol TG(16:0/18:2/20:4)[iso6] without any deuterium labeling in liver samples. 


\subsubsection{Alterations of Hepatic Neutral Lipid and WAT Mass}

To determine an overall change in lipid homeostasis at the liver-WAT axis, neutral lipid in the liver and WAT mass were measured. As shown in Figure 12, oil red O staining of neutral lipid on cryostat liver sections clearly demonstrated accumulation of lipid droplets in the hepatocytes of alcohol-fed mice at 2 weeks. Further increases in number and size of the lipid droplets were observed at 4 weeks. In contrast to neutral lipid accumulation in the liver, WAT mass was significantly lower in the alcohol-fed mice compared to the controls (Figure 13). The weights of both eWAT and sWAT from control mice were increased at 2 weeks and a further increase was found at 4 weeks. However, the weights of both eWAT and sWAT from alcohol-fed mice did not change at either 2 weeks or 4 weeks, leading to an increased difference between the control and alcohol mice along the 4 weeks of experiment. The time course changes in WAT to body weight ratio showed similar trends to that of WAT mass. 

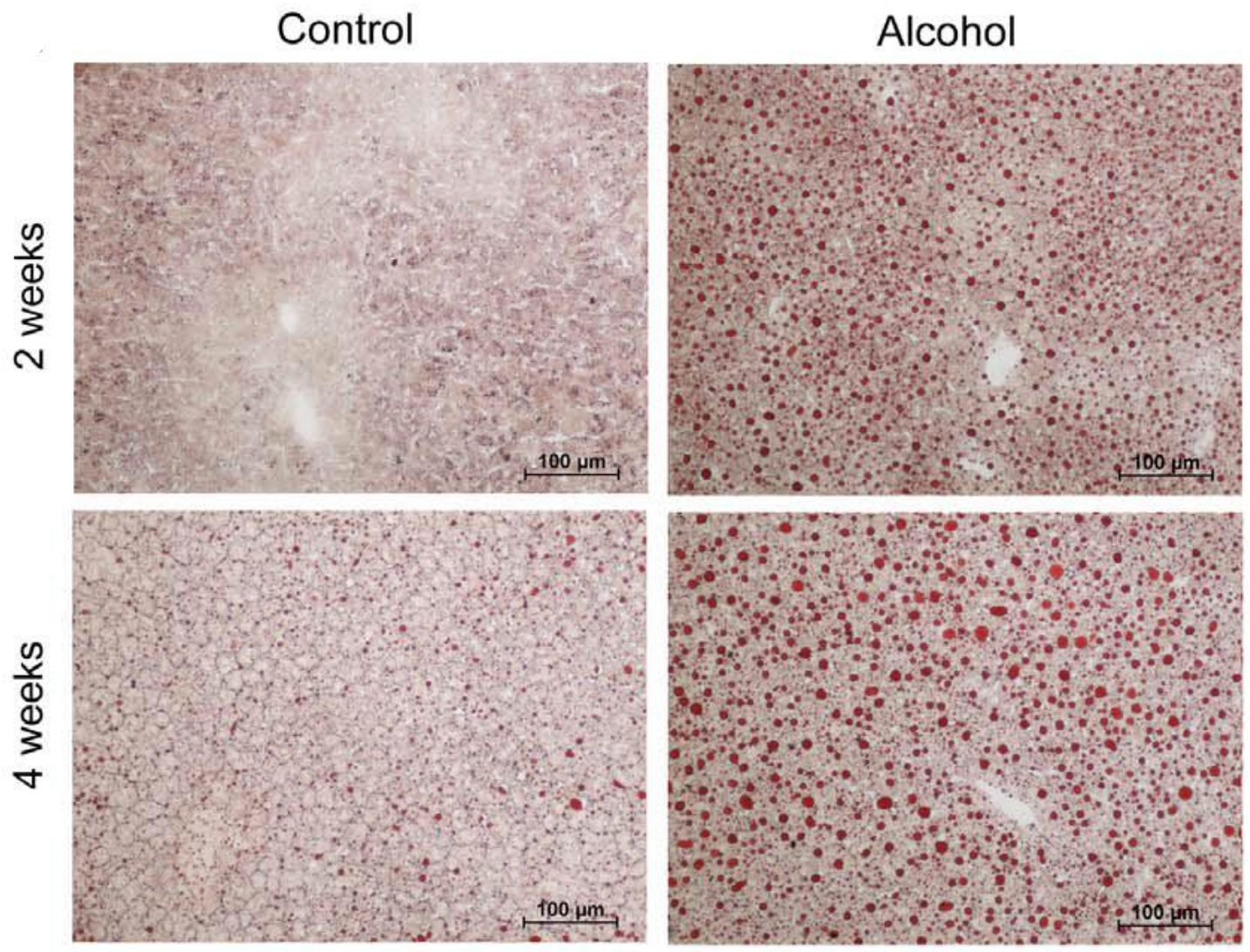

Figure 12. Time course changes of hepatic lipid content. Hepatic neutral lipid detected by Oil red O staining of cryostat liver sections. Alcohol exposure increased hepatic neutral lipid (lipid droplets) gradually along the 4 weeks of experiment. 

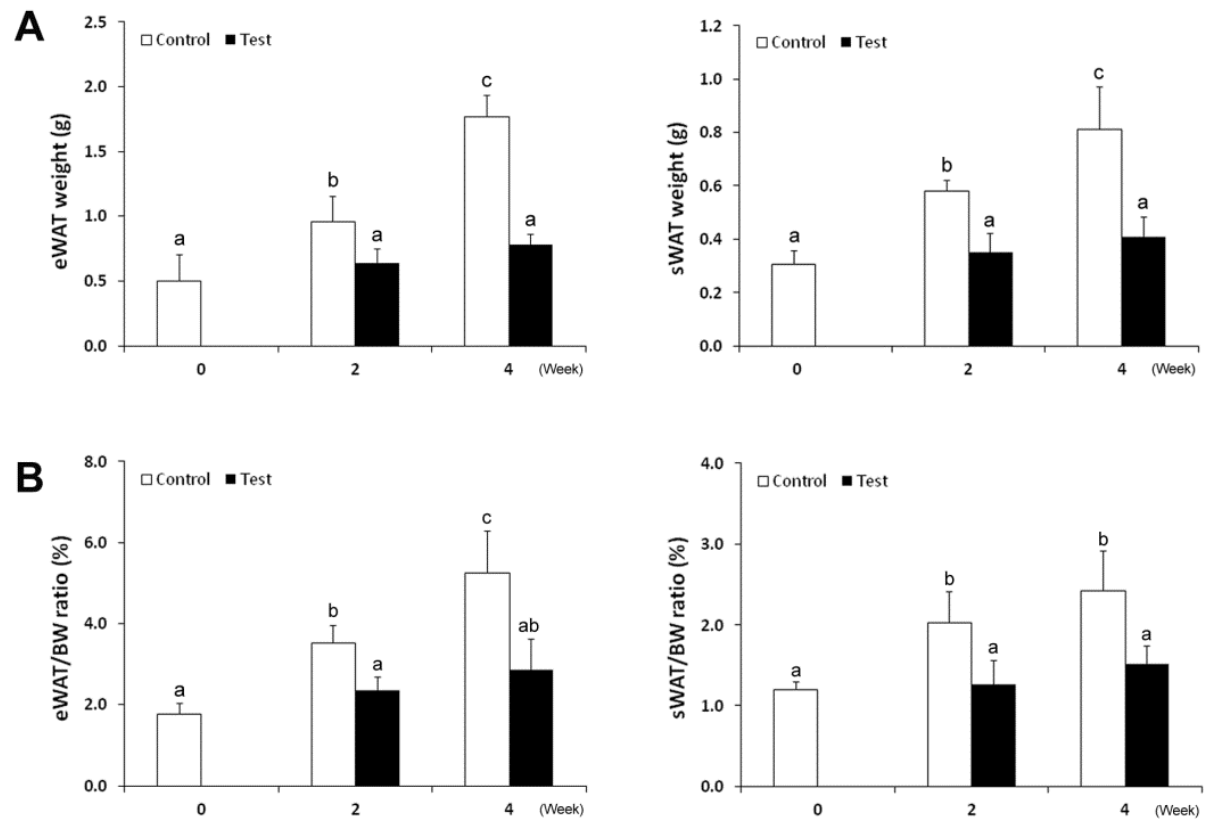

Figure 13. Time course changes of WAT tissues.(A) WAT mass. The weights of both eWAT and sWAT in control mice increased gradually during the 4 weeks of experiment. However, the alcohol-fed mice did not show weight change in both eWAT and sWAT at either 2 weeks or 4 weeks. (B) WAT to body weight ratio (\%). Data are expressed as mean \pm SD (n=6-8). Statistical differences were analyzed by ANOVA followed by Bonferroni post hoc comparison, and means without a common letter differ at $p<0.05$. 
Table 5. List of triacylglycerols in sWAT and eWAT identified with significant concentration changes between the control cohort and the test cohort at two and four weeks.

\begin{tabular}{|c|c|c|c|c|c|c|c|}
\hline WAT & $\begin{array}{l}\text { Time } \\
\text { (Week) }\end{array}$ & $\mathrm{m} / \mathrm{z}$ & p-value & $\begin{array}{l}\text { Fold change } \\
\qquad(\mathrm{T} / \mathrm{C})^{\mathrm{a}}\end{array}$ & Metabolite common name & $\begin{array}{l}\text { Adduct } \\
\text { ion }\end{array}$ & No. ${ }^{2} \mathrm{H}$ \\
\hline \multirow{4}{*}{ sWAT } & 2 & 879.7446 & $2.1 \times 10^{-2}$ & 0.74 & TG(16:1/18:2/20:4)[iso6] & $\mathrm{H}^{+}$ & 2 \\
\hline & 2 & 880.7507 & $3.9 \times 10^{-2}$ & 0.69 & TG(16:0/18:1/18:2)[iso6] & $\mathrm{Na}^{+}$ & 1 \\
\hline & 4 & 826.7013 & $1.7 \times 10^{-3}$ & 0.28 & TG(16:0/16:1/16:1)[iso3] & $\mathrm{Na}^{+}$ & 1 \\
\hline & 4 & 879.7446 & $1.5 \times 10^{-2}$ & 0.74 & TG(16:1/18:2/20:4)[iso6] & $\mathrm{H}^{+}$ & 2 \\
\hline \multirow{10}{*}{ eWAT } & 2 & 826.7017 & $4.1 \times 10^{-4}$ & 0.38 & TG(16:0/16:1/16:1)[iso3] & $\mathrm{Na}^{+}$ & 1 \\
\hline & 2 & 850.7013 & $1.3 \times 10^{-2}$ & 0.68 & TG(16:1/16:1/18:2)[iso3] & $\mathrm{Na}^{+}$ & 1 \\
\hline & 2 & 854.7334 & $2.7 \times 10^{-3}$ & 0.58 & TG(16:0/16:1/20:4)[iso6] & $\mathrm{H}^{+}$ & 1 \\
\hline & 2 & 855.7378 & $1.9 \times 10^{-2}$ & 0.70 & TG(16:0/16:0/18:2)[iso3] & $\mathrm{Na}^{+}$ & 2 \\
\hline & 4 & 828.7174 & $1.6 \times 10^{-2}$ & 0.19 & TG(16:0/16:1/16:1)[iso3] & $\mathrm{Na}^{+}$ & 3 \\
\hline & 4 & 854.7334 & $1.5 \times 10^{-2}$ & 0.54 & TG(16:0/16:1/20:4)[iso6] & $\mathrm{H}^{+}$ & 1 \\
\hline & 4 & 855.7378 & $4.4 \times 10^{-2}$ & 0.48 & TG(16:0/16:0/18:2)[iso3] & $\mathrm{Na}^{+}$ & 2 \\
\hline & 4 & 856.7482 & $2.2 \times 10^{-3}$ & 0.49 & TG(16:0/16:0/18:1)[iso3] & $\mathrm{Na}^{+}$ & 1 \\
\hline & 4 & 879.7445 & $1.5 \times 10^{-2}$ & 0.73 & TG(16:1/18:2/20:4)[iso6] & $\mathrm{H}^{+}$ & 2 \\
\hline & 4 & 880.7498 & $7.7 \times 10^{-3}$ & 0.77 & TG(16:0/18:1/18:2)[iso6] & $\mathrm{Na}^{+}$ & 1 \\
\hline
\end{tabular}

a. Fold-change is the ratio of average peak area of a metabolite in the test cohort (T) to that in the control cohort $(\mathrm{C})$.

\subsubsection{Routine Parameters}

Table 6 listed the results of routine parameters including body weight, liver weight, liver to body weight ratio, and plasma ALT activity and FFA concentration. The test cohort showed a lower body weight but a higher liver weight, leading to a significant increase in liver/body weight ratio at both 2- and 4-week time points. The plasma ALT activity level, an indicator of liver injury, was elevated in the test cohort at both time points. The plasma triacylglycerol level was also increased in the test cohort at 4-week. However, plasma cholesterol and FFAs was not affected by alcohol exposure. 
Table 6. Body weight, liver weight and plasma parameters of the control cohort and the test cohort at two and four weeks.

\begin{tabular}{lccccc}
\hline & \multicolumn{2}{c}{2 weeks } & & \multicolumn{2}{c}{4 weeks } \\
\cline { 2 - 3 } \cline { 5 - 6 } Control & & Test & & Control & Test \\
\hline Body weight $(\mathrm{BW}, \mathrm{g})$ & $28.6 \pm 0.9 \mathrm{a}$ & $26.9 \pm 1.3 \mathrm{a}$ & & $32.4 \pm 2.7 \mathrm{~b}$ & $27.9 \pm 1.2 \mathrm{a}$ \\
Liver weight $(\mathrm{g})$ & $1.06 \pm 0.04 \mathrm{a}$ & $1.16 \pm 0.05 \mathrm{~b}$ & & $1.10 \pm 0.04 \mathrm{ab}$ & $1.32 \pm 0.08 \mathrm{c}$ \\
Liver/BW ratio (\%) & $3.69 \pm 0.22 \mathrm{a}$ & $4.33 \pm 0.30 \mathrm{~b}$ & & $3.41 \pm 0.16 \mathrm{a}$ & $4.77 \pm 0.21 \mathrm{c}$ \\
ALT (U/L) & $20.4 \pm 9.1 \mathrm{a}$ & $48.2 \pm 9.1 \mathrm{~b}$ & & $25.6 \pm 3.1 \mathrm{a}$ & $58.6 \pm 12.6 \mathrm{~b}$ \\
Triacylglycerol (mg/dL) & $93.2 \pm 10.4 \mathrm{a}$ & $130.5 \pm 24.7 \mathrm{ab}$ & & $110.4 \pm 11.3 \mathrm{a}$ & $171.9 \pm 47.7 \mathrm{~b}$ \\
Cholesterol (mg/dL) & $121.7 \pm 19.6$ & $107.1 \pm 14.8$ & & $110.7 \pm 10.3$ & $101.4 \pm 10.3$ \\
FFA (mg/dL) & $0.36 \pm 0.02$ & $0.31 \pm 0.05$ & & $0.34 \pm 0.05$ & $0.33 \pm 0.07$ \\
& & & &
\end{tabular}

Data are expressed as mean \pm SD $(n=6-8)$. Statistical differences were analyzed by ANOVA followed by Bonferroni post hoc comparison, and means without a common letter differ at $p<0.05$.

\subsection{Discussion}

TGs are the group of most abundant lipids in liver, eWAT and sWAT. Analysis of the abundance changes of TGs is enough for us to test our hypothesis. Therefore, the methanol/water phase of metabolite extract from mouse tissues was discarded during the process of metabolite extraction, while the organic phase was used for analysis. It is possible that the regulations of other types of metabolites are also changed besides TGs during the mouse feeding period. However, the changes of these metabolites are not in the scope of this study. 


\subsubsection{Biological Experiment Design}

A two-stage animal feeding experiment was performed in this study to differentiate the hepatic lipids synthesized from the fatty acids transported back from adipose tissue from that synthesized using fatty acids from de novo lipogenesis. It is expected that majority of

the lipids synthesized in the stage-one experiment were incorporated with a certain number of deuterium atoms, and most of them were transported and stored in WAT. The purpose of the stage-two experiment was to induce fatty liver in the test cohort and to use the mice in the control cohort as reference to monitor the lipid concentration change in the mice of the test cohort.

During the stage-two experiment, lipids were continuously synthesized in the mouse livers in both the test and the control cohorts. The lipids synthesized from the uptake of dietary fats should not incorporate any deuterium atoms, except a very small fraction of naturally occurring deuterium in the dietary fats. Therefore, two forms of lipids should be present in mouse liver: deuterium incorporated lipids and non-deuterium incorporated lipids. In case of the control cohort, the deuterium incorporated lipids were synthesized during the stage-one experiment while the non-deuterium incorporated lipids were synthesized in the stage-two experiment.

\subsubsection{Statistical Significance Tests}

Compared to the levels of deuterium incorporated TGs in the control cohort, the significant increase of the deuterium incorporated TGs in the test cohort of liver samples (Table 4) indicates that extra deuterium incorporated fatty acids were used for the synthesis of TGs in alcoholic fatty liver at 2 and 4 weeks. The only source of the extra 
deuterium incorporated fatty acids is the WATs, where the deuterium incorporated fatty acids were stored in the form of TGs during the stage-one experiment. Therefore, a reasonable explanation to the increase of deuterium incorporated TGs in alcoholic fatty liver in the test cohort is that the deuterium incorporated fatty acids were transported back from the WAT after lipolysis due to alcohol consumption. Indeed, such an explanation is further substantiated by the decrease of deuterated TGs in eWAT and sWAT, respectively (Table 5). This supports our hypothesis, i.e., alcohol consumption stimulates lipolysis in the WAT of mice, leading to release of fatty acids, which are transported back and deposited in the liver for the synthesis of TGs.

\subsubsection{Temporal Analysis}

Figures 10A demonstrates that portions of the accumulated TGs in fatty liver were synthesized using deuterium incorporated fatty acids that were transported back from the WAT due to lipolysis, while Figure 10B and 10C demonstrate a significant abundance decrease of the deuterated TGs in eWAT and sWAT, respectively. These results reveal a direct link between WAT fatty acid release and hepatic TG deposition in the development of alcoholic fatty liver. A previous study also demonstrated that diminishing lipid storage function in WAT by over-expressing leptin-receptor b (lpr-b) on the aP2-lpr-b promoter (aP2lepr-b transgene) in $\mathrm{db} / \mathrm{db}$ mice attenuated obesity after high fat feeding[113]. However, the aP2lepr-b transgene significantly increased liver weight and triglyceride concentrations, and accelerated the development of diabetes. Therefore, WAT dysfunction in lipid storage could be an important determinant in the pathogenesis alcoholic or nonalcoholic fatty liver. 
Figure 11 displays the time course trajectory of triacylglycerol TG(16:0/18:2/20:4)[iso6] without any deuterium labeling in liver samples. This TG molecule is synthesized by using fatty acids from dietary source and/or hepatic de novo lipogenesis rather than by using fatty acids transported back from the WAT. The time dependent abundance of this metabolite in the test sample is always higher than its abundance in the control sample at both 2 weeks and 4 weeks. These data indicate that fatty acids from the WAT are not the sole source of TG synthesis, and fatty acids from diet and/or de novo synthesis also contribute to the development of alcoholic fatty liver.

\subsubsection{WAT Dysfunction and Fatty Liver}

Time course changes in WAT weight (Figure 13) demonstrated that WAT mass in control mice significantly increased along the 4 weeks of feeding. Surprisingly, the WAT mass of alcohol fed mice did not changed at either 2 weeks or 4 weeks compared to time 0 , indicating a loss of the lipid storage function. A previous study has reported that alcohol feeding to rats reduced total body fat content due to an increase of TG turnover in rats, as indicated by a 2.3 -fold increase in TG degradation with no significant change in TG synthesis[102]. Our previous report also showed that alcohol exposure activates lipolysis pathways in WAT, thereby accelerating fatty acid release[105]. Adipose lipolysis is regulated positively by catecholamine and negatively by insulin[114]. Previous studies suggested that alcohol-increased lipolysis is most likely through disturbing insulin signaling rather than enhancing catecholamine-mediated lipolysis[102,105,115]. Insulin negatively regulates lipolysis, and phosphodiesterase 4 (PDE4) and activating protein phosphatase 1 (PP1) mediate insulin signaling via reducing cellular cAMP level and dephosphorylating hormone sensitive lipase (HSL), 
respectively[114]. Although the adipose PDE4 was not affected in the WAT of alcohol-fed rats[102], our previous study showed that PP1 protein level was reduced in the WAT of alcohol-fed mice[105]. We also found that chronic alcohol exposure up-regulated negative regulators of insulin signaling, including phosphatase and tensin homolog (PTEN) and suppressor of cytokine signaling 3 (SOC3). In addition to lipid storage dysfunction, alcohol exposure also inhibited expression and secretion of adipokines including adiponectin and leptin in WAT[116-118]. Both adiponectin and leptin critically modulate hepatic lipid metabolism toward reduction of lipid content in the liver. Normalizing plasma adiponectin or leptin level was associated with attenuation of alcoholic fatty liver[119-121]. Therefore, adipose tissue dysfunction may contribute to the development of alcoholic fatty liver by directly supplying fatty acids for hepatic TG synthesis or indirectly disturbing adipokine regulation of hepatic lipid metabolism.

\subsubsection{Alcohol-induced Hepatic Lipid Dyshomeostasis}

Alcohol exposure may disturb hepatic lipid metabolism in multiple pathways, including fatty acid uptake, fatty acid oxidation, de novo lipogenesis and lipid export[116,122]. The present study demonstrated that alcohol exposure causes a reverse transport of TGs from WAT to the liver. Liver plays a central role in lipid metabolism, but it does not store lipid at physiological condition. Balance between TG synthesis and export is a key determent of hepatic lipid homeostasis[123,124]. Fatty acids from either blood or de novo synthesis are converted to TGs which are exported to the blood in the form of very low density lipoproteins (VLDL) for use or storage by the peripheral organs. Even though alcohol induces hepatic influx of fatty acids, fatty liver should not be developed as long as the liver can efficiently secrete TGs into the blood. Therefore, impaired VLDL 
secretion should co-exist with hepatic fatty acid influx in the development of alcoholic fatty liver. The present study shows that the liver of alcohol-fed mice accumulated TGs synthesized by using fatty acids of both deuterium labeled from WAT source and non-deuterium labeled from dietary fats or de novo lipogenesis. These data suggest that alcohol blunted lipid export. The authors believe that the increased blood TG levels in the test cohort at 4 weeks may indicate an impaired TG uptake from VLDL in WATs, rather than an increased hepatic TG secretion. Indeed, our previous study demonstrated that alcohol exposure significantly reduced the rate of VLDL-TG secretion from the liver to the blood[104, 106]. Disruption of VLDL secretion is likely an important mechanism underlying alcoholic fatty liver, because improvement of VLDL secretion was associated with attenuation of alcoholic fatty liver by zinc, betaine or hepatocyte growth factor[104,125,126]. Further investigations are required to determine the mechanisms of how alcohol exposure suppresses lipid export function of the liver.

\subsection{Conclusions}

We used an analytical method of employing high-resolution mass spectrometry in combination with metabolite deuterium labeling for the analysis of triacylglycerol. A two-stage mouse feeding schema was designed, where all mice were first fed with deuterated water to label WAT TGs (stage one), followed by pair-feeding an alcohol or isocaloric maltose dextrin control liquid diet for two or four weeks. Hepatic lipids extracted from the livers, eWAT and sWAT tissues were detected by FTICR-MS and LTQ-MS/MS. All observations in this study, including the increase of TGs in the test cohort of liver, the simultaneous decrease of TGs in the test cohort of eWAT and sWAT, and the agreement between the metabolomics data and the histological data demonstrate 
that chronic alcohol exposure disturbs lipid homeostasis at the adipose tissue-liver axis and therefore, support our hypothesis, that is, alcohol consumption stimulates lipolysis of the WAT and leads to an excess release of fatty acids which are transported to the liver and deposited as TGs. Furthermore, accumulation of TGs synthesized from fatty acids from dietary source or de novo lipogenesis also contributes to the pathogenesis of fatty liver. 


\section{CHAPTER 4}

\section{THE EFFECTS OF CHRONIC ARSENIC EXPOSURE IN A MOUSE MODEL OF DIET-INDUCED FATTY LIVER DISEASE}

\subsection{Introduction}

Diet-environment interactions play an important role in the development and progression of many human diseases, including fatty liver disease (FLD). According to the prevailing two hit hypothesis[127], dietary factors such as excessive fat consumption contribute to the formation of fatty liver, which makes the liver susceptible to a second hit which promotes the progression to more severe liver injury. Several factors have been shown to mediate the progression from simple steatosis (fatty liver) to steatohepatitis (fatty liver with inflammation and cell death)[128] and environmental agents that activate these factors can contribute to the extent of liver injury[129,130]. For example, arsenic was recently shown to contribute to the progression of diet-induced fatty liver disease by increasing inflammatory mediators in mouse liver[131].

Although several regions in the United States where municipal water supplies and private artesian wells have arsenic concentrations exceeding the Maximum Contaminant Level of 10 parts per billion (ppb) set by the World Health Organization and the Environmental Protection Agency, arsenic concentrations can reach much higher concentrations in places like Bangladesh and West Bengal[132]. Epidemiological studies in these 
highly-exposed populations have revealed strong links between arsenic exposure and skin lesions, hypertension, cardiovascular disease, respiratory disease, and cancer[133]. In the liver, high arsenic exposure results in hepatomegaly, portal hypertension, non-cirrhotic portal fibrosis and cancer[134]. It is unclear whether the liver is a primary target of toxicity in people exposed to lower levels of arsenic, such as those seen in the United States. It is more likely that the adverse effects of arsenic on the liver will depend on the presence of other sensitizing factors like fatty liver disease.

It has been reported that mice fed a diet high in fat and cholesterol (the so-called Western Diet) for 10 weeks developed steatosis, while simultaneous exposure to drinking water contaminated with arsenic resulted in much more inflammation and cell death[131]. In that study, 5 parts per million (ppm) arsenic had no effect on the livers of mice fed a low fat control diet. While this concentration is higher than those to which produce hepatotoxicity in human populations[134] the absorption, distribution and metabolism of arsenic is much different in mice than in humans. Indeed, a recent report showed that it took 10 times higher concentrations of drinking water (arsenic $50 \mathrm{ppm}$ ) to achieve liver arsenic concentrations similar to those seen in humans exposed to arsenic in West Bengal [135].

To better understand the interactions between arsenic and diet-induced fatty liver disease, we employed high throughput comprehensive two-dimensional gas chromatography time-of-flight mass spectrometry (GC×GC-TOF MS) to analyze liver metabolites altered by arsenic, a high fat diet, and the combination of the two. GC $\times \mathrm{GC}-\mathrm{TOF}$ MS is one of the most powerful analytical platforms for metabolomics analyses. It is an emerging technology that provides considerably more chemical selectivity than GC-MS. It uses two 
capillary GC columns connected via a thermal modulator to achieve a high degree of separation of metabolites[136-138]. Compared to the 15-60 m length of the first dimension column, the length of the second dimension column is very short, in general only $0.5-2 \mathrm{~m}$. These two GC columns usually have different stationary phases to maximize the orthogonality of the two-dimensional separation system to achieve high peak capacity. The analytes co-eluted from the first column are further separated in the second column because of the difference of column temperature and the chromatographic polarities. The further separated analytes are directed to a high capacity time-of-flight mass spectrometry system for detection. The GC $\times$ GC-TOF MS platform offers significant advantages over other metabolomics platforms for analysis of complex samples, including an order-of-magnitude increase in separation capacity, significant increase in signal-to-noise ratio and dynamic range, and improvement of mass spectral deconvolution and similarity matches[139,140]. Presently, GC×GC-MS has not been widely used for large-scale broad-spectrum biomarker discovery most likely due to the increased difficulty of data analysis. New bioinformatics tools have recently emerged that greatly decrease the personnel time necessary to properly process the GC $\times \mathrm{GC}-\mathrm{MS}$ data[141-143] making this approach feasible for large-scale metabolomics studies[3, 5]. Our in-house developed bioinformatics tools MetPP combined with GC×GC-MS bioanalytical platform, were used here. 


\subsection{Experimental}

\subsubsection{Materials and Reagents}

Chemicals used in this experiment include organic solvents (methanol, chloroform and pyridine), amino acids (glycine, L-serine, L-proline, L-phenylalanine, L-threonine, L-methionine, L-aspartic acid, L-glutamic acid, L-lysine, L-histidine, and L-alanine), organic acids (adipic acid, fumaric acid, malonic acid, oxalic acid, succinic acid), fatty acids (butyric acid, dodecanoic acid, hexadecanoic acid, heptadecanoic acid, heptanoic acid, nonanoic acid, octadecanoic acid, pentadecanoic acid, tetradecanoic acid, tridecanoic acid), oximation and derivatization reagents (tert-butyldimethylchlorosilane (TBDMSCI) and N-tert-butyldimethylsilyl-N-methyltrifluoroacetamide (MTBSTFA)). All of these chemicals were purchased from Sigma-Aldrich Corp. (St. Louis, MO, US). A deuterated semi-volatile internal standard (ISTD) mixture containing acenaphthene-d10, chrysene-d12, 1,4-dichlorobenzene-d4, naphthalene-d8, perylene-d12, phenanthrene-d10 was purchased from Restek Corp. (Bellefonte, PA, US)

\subsection{2. $\quad$ Spiked-in Samples}

To extract metabolites from mouse liver, a $200 \mu \mathrm{L}$ aliquot of homogenized liver sample was mixed with $800 \mu \mathrm{L}$ of methanol and vortexed for $1 \mathrm{~min}$, followed by centrifugation at $4{ }^{\circ} \mathrm{C}$ for $10 \mathrm{~min}$ at $15,000 \mathrm{rpm} .0 .8 \mathrm{~mL}$ of the top solution was aspirated into a plastic tube and dried by $\mathrm{N}_{2}$ flow. After dissolving the dried sample with $100 \mu \mathrm{L}$ of pyridine, $100 \mu \mathrm{L}$ of MTBSTFA was added and the derivatization was lasted for 1 hour at $70{ }^{\circ} \mathrm{C}$.

A mixture of 28 acid standards was prepared at a concentration of $100 \mu \mathrm{g} / \mathrm{mL}$ per acid. The acids included glycine, L-alanine, L-proline, L-cystine, L-histidine, L-phenylalanine, 
L-tyrosine, L-lysine, L-glutamic acid, L-methioneine, L-proline, L-serine, L-threonine, L-tryptophan, L-valine, L-leucine, adipic acid, butyric acid, fumaric acid, malonic acid, oxalic acid, succinic acid, dodecanoic acid, docosanoic acid, hexadecanoic acid, heptadecanoic acid, octadecanoic acid, tetradecanoic acid, nonadecanoic acid, tridecanoic acid, hepadecanoic acid, heptanoic acid, nonanoic acid and pentadecanoic acid. $100 \mu \mathrm{L}$ of the acid mixture was added to $100 \mu \mathrm{L}$ MTBSTFA at $70{ }^{\circ} \mathrm{C}$ and kept 1 hour for derivatization.

Ten $\mu \mathrm{L}$ of the acid mixture was added to the first vial, while $20,40,50$ and $80 \mu \mathrm{L}$ of the acid mixture were added to the other vials, respectively. Twenty $\mu \mathrm{L}$ of derivatized liver extract sample and $5 \mu \mathrm{L}$ of ISTD at a concentration of $200 \mu \mathrm{g} / \mathrm{mL}$ were added to each of the five vials, respectively. Pyridine was then added to each of the 5 vials to make the total volume of each aliquot to $105 \mu \mathrm{L}$. This resulted in five samples with spiked-in acid standards. The acid concentration of the amount of spiked-in acid in the 5 samples is approximately $6.4 \mu \mathrm{g} / \mathrm{mL}, 12.8 \mu \mathrm{g} / \mathrm{mL}, 25.6 \mu \mathrm{g} / \mathrm{mL}, 32.0 \mu \mathrm{g} / \mathrm{mL}$ and $51.2 \mu \mathrm{g} / \mathrm{mL}$, respectively. The ratio of the concentrations is $1: 2: 4: 5: 8$ in terms of the amount of spiked-in standards. A blank sample was also prepared in parallel without adding acid standards and liver extract. Each of the five samples was injected 6 times on the GC×GC-TOF MS instrument to generate one sample group, respectively.

Of these 28 acids, 23 of them already exist in mouse liver including adipic acid, fumaric acid, malonic acid, oxalic acid, dodecanoic acid, hexadecanoic acid, heptadecanoic acid, decanoic acid, nonanoic acid, octadecanoic acid, pentadecanoic acid, tetradecanoic acid, glycine, L-alanine, L-glutamic acid, L-leucine, L-lysine, L-methionenine, L-phenylanine, L-proline, L-serine, L-threonine, and L-valine. The concentration ratio of these metabolites 
among the five sample groups is not 1:2:4:5:8, due to the presence of these metabolites in the mouse liver extract.

\subsubsection{Animals and Diets}

Four week old male C57Bl/6J mice were purchased from The Jackson Laboratory (Bar Harbor, ME). Mice were housed in a pathogen-free barrier facility accredited by the Association for Assessment and Accreditation of Laboratory Animal Care. Food and tap water were allowed ad libitum. The procedures of animal care were approved by the University of Louisville Institutional Animal Care and Use Committee.

All mice were fed AIN-76A Purified diet (Harlan Laboratories, Madison, WI) for one week to minimize the potential confounding factor of arsenic present in standard laboratory chow [144]. Mice were exposed to sodium arsenite (5 ppm in tap water) or tap water for one week prior to initiating feeding with either low fat diet (13\% of calories from fat) or high fat diet (42\% of calories from fat) (diets TD.08485 and TD.88137, Harlan Laboratories, Madison, WI) for 10 weeks. This exposure level of arsenic was shown in literature[131] to cause no overt liver damage in mice fed low-fat diet, but to exacerbate high fat diet-induced liver injury. Four different treatment groups were evaluated in this study: 6 mice fed a low fat diet and tap water (sample group LFD); 6 mice fed a low fat diet and tap water containing sodium arsenite (sample group LFD+As); 5 mice fed a high fat diet and tap water (sample group HFD); 6 mice fed a high fat diet and tap water containing sodium arsenite (sample group HFD+As). Food and water consumption were measured twice a week. Body weight was measured once a week. For termination, mice were anesthetized with ketamine/xylazine (100/15 mg/kg i.m.). Portions of liver tissue were frozen immediately in liquid nitrogen. 


\subsubsection{Metabolite Sample Preparation from Mouse Liver}

The liver tissue of each mouse was weighed and then homogenized for 2 min after adding water at a ratio of $100 \mathrm{mg}$ liver tissue $/ \mathrm{mL}$ water. The homogenized sample was then stored at $-80{ }^{\circ} \mathrm{C}$ until use. A $100 \mu \mathrm{L}$ aliquot of the homogenized liver sample and $400 \mu \mathrm{L}$ methanol were mixed and vortexed for 1 min followed by centrifugation at room temperature for $10 \mathrm{~min}$ at $15000 \mathrm{rpm} .400 \mu \mathrm{L}$ of the supernatant was aspirated into a plastic tube and dried by $\mathrm{N}_{2}$ flow. The metabolites extracts were then dissolved in $40 \mu \mathrm{L}$ ethoxyamine hydrochloride solution $(30 \mathrm{mg} / \mathrm{mL})$ and vigorously vortex-mixed for $1 \mathrm{~min}$. Methoxymation was carried out at $70{ }^{\circ} \mathrm{C}$ for 1 hour. After adding $40 \mu \mathrm{L}$ MTBSTFA mixed with $1 \%$ TBDMSCI, derivatization was carried out at $70{ }^{\circ} \mathrm{C}$ for 1 hour. Stock solutions were then transferred to GC vials for analysis. The methoxymation and derivatization were prepared just before $\mathrm{GC} \times \mathrm{GC}-\mathrm{TOF} \mathrm{MS}$ analysis.

\subsection{5. $\mathrm{GC} \times \mathrm{GC}-\mathrm{TOF} \mathrm{MS}$ Analysis}

The LECO Pegasus 4D GC×GC -TOF MS instrument was equipped with an Agilent 6890 gas chromatograph and a Gerstel MPS2 auto-sampler (GERSTEL Inc., Linthicum, MD), featuring a LECO two-stage cryogenic modulator and secondary oven. The primary column was a $60 \mathrm{~m} \times 0.25 \mathrm{~mm}{ }^{1} d_{c} \times 0.25 \mu \mathrm{m}{ }^{1} d_{f}$, DB-5ms GC capillary column (phenyl arylene polymer virtually equivalent to a (5\%-phenyl)-methylpolysiloxane). A second GC column of $1 \mathrm{~m} \times 0.25 \quad \mathrm{~mm} \quad{ }^{1} d_{c} \times 0.25 \quad \mu \mathrm{m} \quad{ }^{2} d_{f}, \quad$ DB17ms ((50\%-phenyl)-methylpolysiloxane) was placed inside the secondary GC oven after the thermal modulator. Both columns were obtained from Agilent Technologies (Agilent Technologies J\&W, Santa Clara, CA). The helium carrier gas (99.999\% purity) flow rate 
was set to $1.0 \mathrm{~mL} / \mathrm{min}$ at a corrected constant flow via pressure ramps. The inlet temperature was set at $280{ }^{\circ} \mathrm{C}$. The primary column temperature was programmed with an initial temperature of $60{ }^{\circ} \mathrm{C}$ for $0.5 \mathrm{~min}$ and then ramped at $5{ }^{\circ} \mathrm{C} / \mathrm{min}$ to $280{ }^{\circ} \mathrm{C}$ and maintained for $12 \mathrm{~min}$. The secondary column temperature program was set to an initial temperature of $70{ }^{\circ} \mathrm{C}$ for $0.5 \mathrm{~min}$ and then also ramped at the same temperature gradient employed in the first column to $280{ }^{\circ} \mathrm{C}$, accordingly. The thermal modulator was set to $+20{ }^{\circ} \mathrm{C}$ relative to the primary oven, and a modulation time of $P_{M}=2.5 \mathrm{~s}$ was used. The mass range was set as $45-1000 \mathrm{~m} / \mathrm{z}$ with an acquisition rate of 200 mass spectra per second. The ion source chamber was set at $230{ }^{\circ} \mathrm{C}$ with the transfer line temperature of $280{ }^{\circ} \mathrm{C}$, and the detector voltage was $1680 \mathrm{~V}$ with electron energy of $70 \mathrm{eV}$. The acceleration voltage was turned on after a solvent delay of $775 \mathrm{~s}$. The split ratio was set at 40:1.

\subsubsection{Data Analysis}

The GC $\times$ GC-TOF MS data were processed using LECO's instrument control software ChromaTOF for peak picking and tentative metabolite identification, followed by retention index matching, peak merging, peak list alignment, normalization, and statistical significance test. For metabolite identification using ChromaTOF, each chromatographic peak was tentatively assigned to a metabolite if its experimental mass spectrum and a database spectrum have a spectral similarity score no less than 600 . Note that the maximum spectral similarity score is 1000 . Peak merging and peak list alignment were carried out using DISCO software[17], while the retention index matching was performed using iMatch software with the $p$-value threshold set as $p \leq 0.001[18]$. The pairwise two-tail $t$-test was used to determine whether a metabolite has a significant 
abundance difference between sample groups by setting the threshold of false discovery rate $q \leq 0.2$.

To further verify the identification of metabolites detected with significant abundance difference between sample groups, commercially available authentic standards of these metabolites were analyzed on $\mathrm{GC} \times \mathrm{GC}-\mathrm{TOF}$ MS under the same experimental conditions as the biological samples analyzed. A tentative metabolite assignment was considered as a correct identification only if the experimental information of the authentic metabolite agreed with the corresponding information of the chromatographic peak in the biological samples, i.e., difference of the first dimension retention time $\leq 10 \mathrm{~s}$, difference of the second dimension retention time $\leq 0.06 \mathrm{~s}$, and the mass spectral similarity $\geq 700$.

\subsubsection{Metabolite Relative Quantification}

To measure the accuracy of recognizing the spiked-in metabolites from the experimental data, the receiver operating characteristic (ROC) curve is applied, which is created by plotting the true positive rate (TPR) vs. the false positive rate (FPR) at various threshold settings. TPR, PPV and their harmonic mean F1 score are calculated as follows:

$$
\begin{gathered}
T P R=\frac{T P}{T P+F N} \\
P P V=\frac{T P}{T P+F P} \\
F 1=\frac{2 \times T P R \times P P V}{T P R+P P V}
\end{gathered}
$$


where TP (true-positive) is the number of spiked-in metabolites that were detected as molecules with significant peak area changes between two testing sample groups by statistical analysis, FP (false-positive) is the number of molecules that were not the spiked-in metabolites but detected as molecules with significant peak area changes, and FN (false-negative) is the number of spiked-in metabolites that were not detected as molecules with significant peak area changes. TPR is called recall and PPV precision and their harmonic mean $F 1$ score can be used as an accuracy of the statistical significant test. Furthermore, the area under curve (AUC) in the ROC curve is equal to the probability of positive outcomes being higher than the negative ones. The higher the AUC score is, the better the observed accuracy of the test for statistical significance.

\subsection{Results}

Figure 14 depicts the analysis workflow of this study. Metabolites were first extracted from mouse liver using solvent of methanol and water. The extracted metabolites were then analyzed on GC×GC-TOF MS instrument after derivatization. A suite of bioinformatics tools were then employed for metabolite identification, quantification and pathway analysis.

GC $\times$ GC-TOF MS instrument data provide four pieces of information for each metabolite,

the first dimension retention time ${ }^{1} t_{R}$, the second dimension retention time ${ }^{2} t_{R}$, fragment ion $\mathrm{m} / \mathrm{z}$, and peak height of each fragment ion. The information of fragment ion $\mathrm{m} / \mathrm{z}$ and peak height forms the mass spectrum of the metabolite. Figure 15 is a contour plot of the GC $\times$ GC-TOF MS data acquired from a sample randomly selected from the sample group LFD. The color of each data point is the signal intensity. 


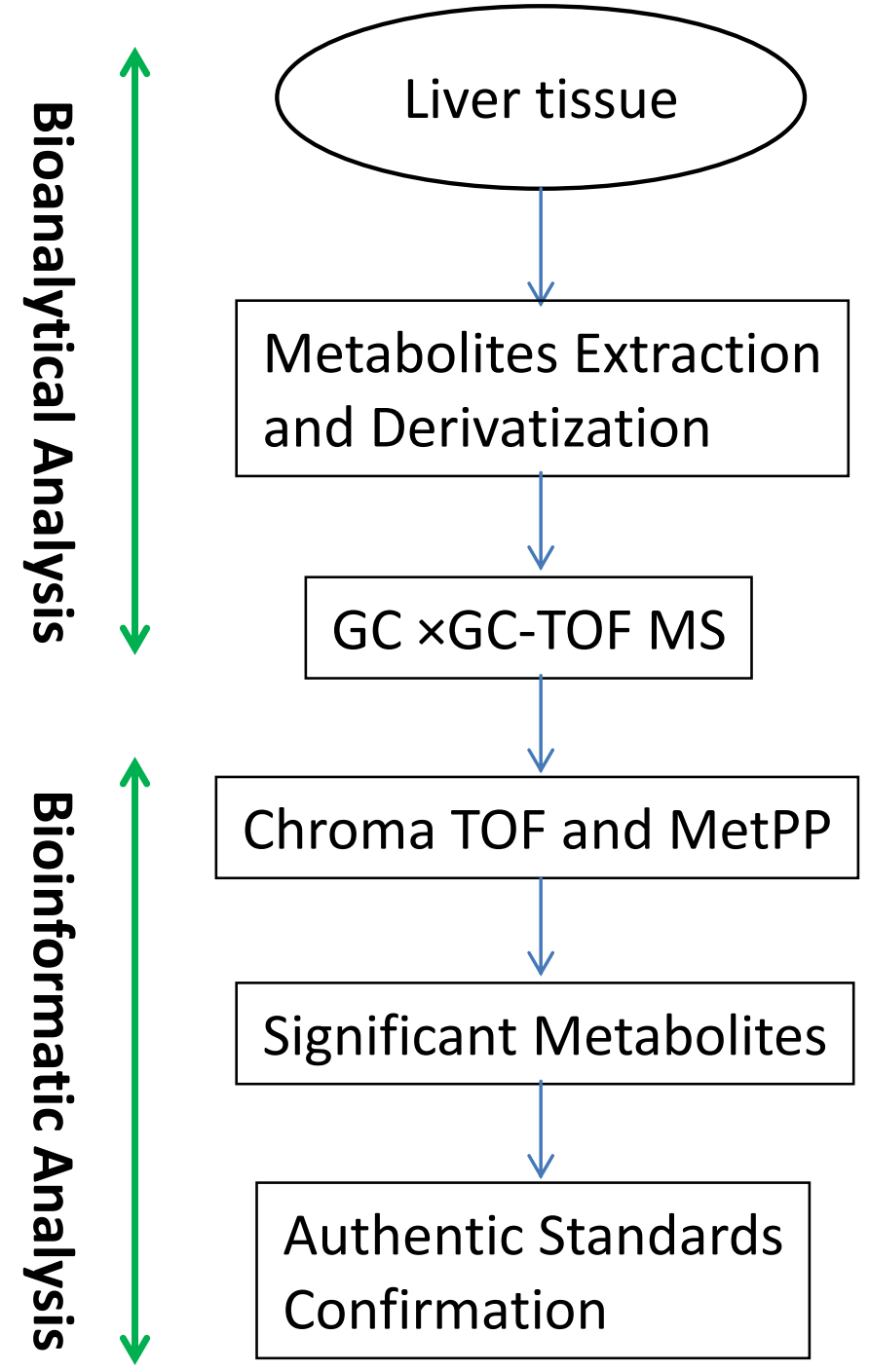

Figure 14. Workflow of metabolomics Study by GC×GC-TOF MS. 


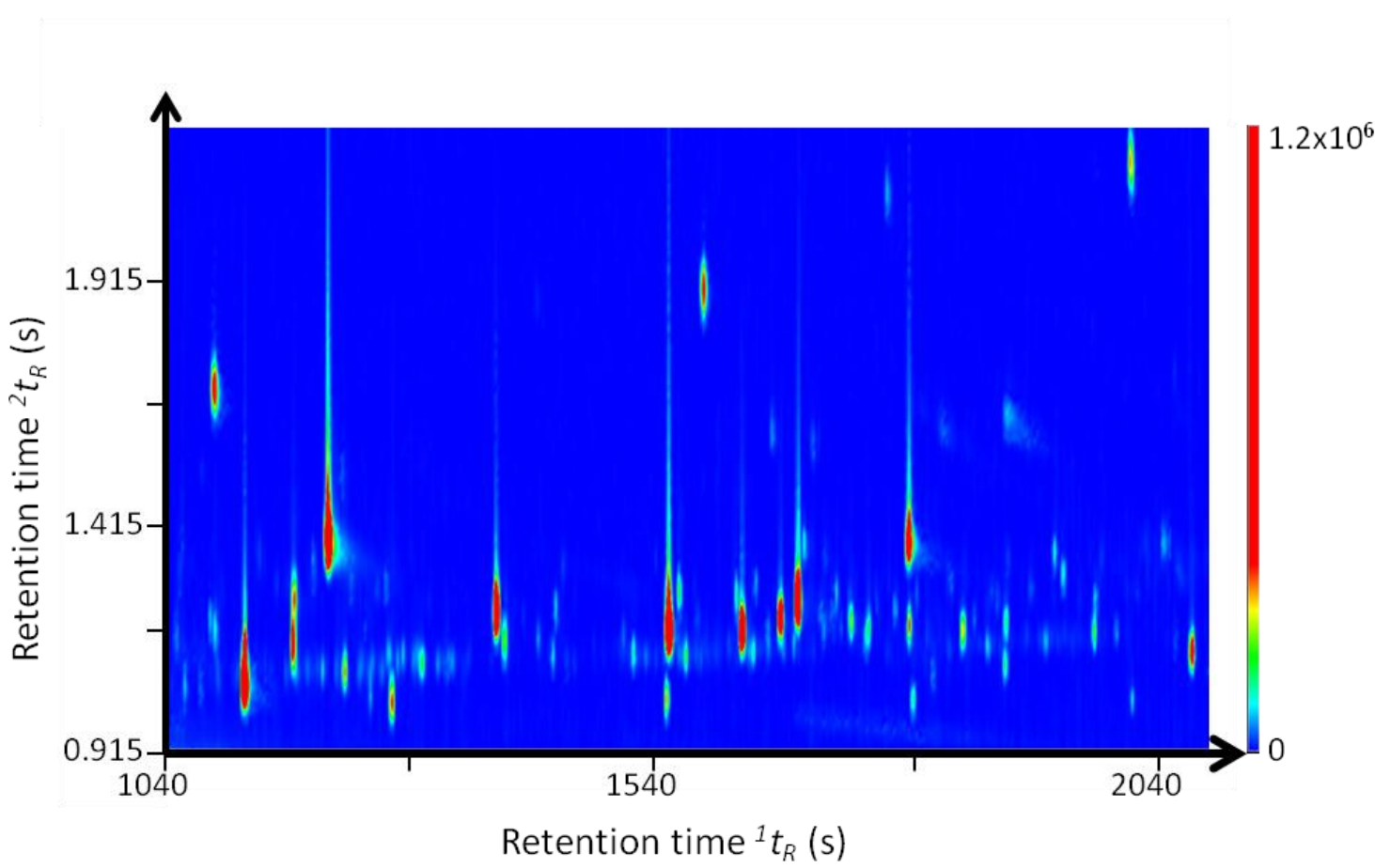

Figure 15. Sample GC $\times$ GC-TOF MS chromatograms of metabolite extract from mouse liver. The $\mathrm{x}$-axis is the first dimension retention time ${ }^{1} t_{R}$ in seconds. The $\mathrm{y}$-axis is the second dimension retention time ${ }^{2} t_{R}$ in seconds. The color bar shows the signal intensity of each peak plotted on the chromatogram in total ion current.

The instrument data were first processed using commercial software ChromaTOF for peak picking and initial compound identification using the vendor recommended parameters, and the information of the top 1500 abundant chromatographic peaks were reported. By setting the threshold of spectral similarity $\geq 600$, about $570-730$ chromatographic peaks were assigned to compounds in each sample, while the remaining chromatographic peaks were assigned as unknowns. The average peak area of the chromatographic peaks with initial identification results is about 6 times larger than the average peak area of the chromatographic peaks assigned as unknowns. The chromatographic peaks with positive identifications were further subjected for retention 
index matching using iMatch algorithm. By setting the threshold $\mathrm{p} \leq 0.001$, about 380-520 compounds had correct retention index values in each sample and the remaining chromatographic peaks were re-assigned as unknowns. All retention index matched compounds were used for alignment using DISCO algorithm. A total of 402 peaks were aligned. The aligned metabolites were further filtered by removing compounds detected in the blank samples as well as the compounds that detected in less than $75 \%$ of samples in each sample group. By doing so, about 100 metabolites left between two sample groups for statistical significance test and FDR analysis.

The ability of the platform to accurately identify metabolites with significant abundance differences between two sample groups was assessed by spiking different concentrations of a mixture of standards into a sample. Figure 17 shows the receiver operator curves produced by these spike-in experiments. A large value of area under curve (AUC) in the receiver operating characteristic (ROC) curve demonstrates that the analytical platform and the data analysis methods used in this study are effective in recognizing the metabolites with significant differences.

\subsubsection{Metabolite Identification}

Metabolite identification was done in three sequential steps in this study: mass spectral matching, retention index matching, and comparison with authentic standards. It is common that multiple distinct chromatographic peaks can be assigned to the same metabolite by mass spectral matching, due to limited accuracy of the identification algorithm[139,140]. For example, glycine, N-(tert-butyldimethylsilyl)-, tert-butylsimethylsilyl ester was assigned to three distinct chromatographic peaks with two-dimensional retention times of (1655 s, $1.155 \mathrm{~s}),(1845 \mathrm{~s}, 1.055 \mathrm{~s})$ and $(1850 \mathrm{~s}, 1.045$ 
s), respectively. The spectral similarity of these three assignments to the mass spectra in the NIST11 database was 872,731 and 787 , respectively. In order to reduce the rate of false identification, we employed a second round of retention index matching to remove the false positive identifications. By setting the threshold $p \leq 0.001$ in iMatch software, the chromatographic peaks with the two-dimensional retention time values of (1845 s, $1.055 \mathrm{~s})$ and $(1850 \mathrm{~s}, 1.045 \mathrm{~s})$ were recognized as false identifications and excluded from the downstream analysis, due to large retention index difference.

Metabolite identifications based on retention index matching were further confirmed by comparison to authentic standards. For example, glycine was detected as a metabolite with significant abundance changes between different sample groups. The retention time values for the metabolite identified as glycine in the biological samples were (1655 s, $1.155 \mathrm{~s})$. The authentic standard of glycine that was derivatized and analyzed on GC $\times$ GC-TOF MS under the same conditions as the biological samples eluted at ${ }^{l} t_{R}=$ $1655 \mathrm{~s}$ and ${ }^{2} t_{R}=1.165 \mathrm{~s}$, which is very similar to the peaks eluted in the biological sample with identical ${ }^{1} t_{R}$ and a difference of $0.01 \mathrm{~s} \mathrm{in}{ }^{2} t_{R}$. The mass spectral similarity between the authentic standards and the biological sample was 970, demonstrating the correct identification of glycine from the biological samples.

Some metabolites tentatively assigned by mass spectrum matching and retention index filtering were not compared to authentic standards because they were not available in our laboratory. Because this technical verification was not performed, their identification remained tentative. Tentatively-identified metabolites were considered as false identifications and removed from the identification list, if they were recognized as drugs or from non-mammalian animals during metabolic network analysis by the Ingenuity 
Pathway Analysis (IPA) software (Ingenuity Systems, Inc., Redwood City, CA). The remaining tentatively-identified metabolites were included in subsequent analyses, but they have been labeled as tentative in Tables 7 through 9.

\subsubsection{Metabolite Quantification}

About 500-800 metabolites were identified in each mouse liver sample via mass spectral matching. By setting $\mathrm{p} \leq 0.001,18.6 \%$ of the identified metabolites were confirmed by the first dimension retention index matching and $51.8 \%$ were preserved due to the lack of retention index information in the NIST11 database. However, $29.5 \%$ of the mass spectral matched metabolites were removed due to the large first dimension retention index deviation from the database values. The mean absolute deviation between the database value and experimental retention index of the removed metabolites is 318 i.u. with a standard deviation of 267 i.u. The corresponding values of the preserved metabolites are $21 \pm 16$ i.u.

After the retention time filtering, about 300-500 peaks were left in each sample for alignment. Among the aligned peaks, the maximum value of the RSD for the first dimensional retention time and the second dimensional retention time was only $3.15 \%$ and $3.30 \%$, respectively.

To study the abundance change of a metabolite between two testing sample groups, the pairwise two-tail $t$-test was employed by setting the threshold of false discovery rate as $q$ $\leq 0.2$. A term fold-change was defined as the ratio of the large abundance value (peak area) of a metabolite in one group divided by the small abundance value of the same metabolite in the other group. A positive sign and a negative sign indicate the abundance 
increase and decrease in the testing sample group compared to the reference sample group, respectively.

Figure 16 depicts a sample abundance distribution of metabolite glycine in sample groups HFD and HFD+As. Glycine was detected by the statistical significance test as one of the metabolites that have significant abundance changes between these two sample groups. Compared with its abundance in the HFD group, glycine is 1.7 -fold lower in the HFD+As group with a $p$-value of $5.0 \times 10^{-3}$.

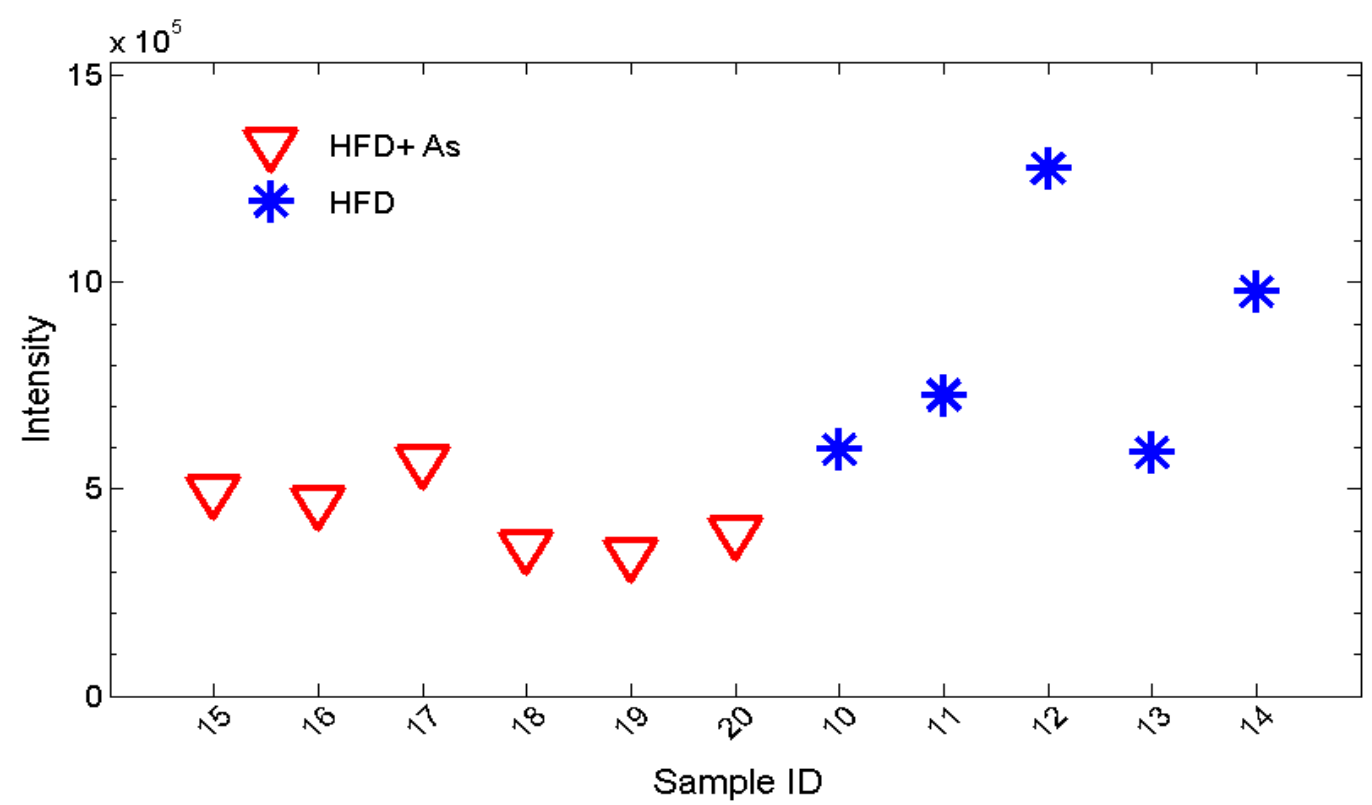

Figure 16. Abundance distribution of metabolite glycine in the samples of HFD+As group and HFD group.The abundance test (pairwise two-tail t-test) shows that the regulation of this metabolite in the HFD+As group is decreased with a fold change of 1.7 and a p-value of $5.0 \times 10^{-3}$ comparing with HFD group.

Mice fed a high fat diet develop steatotosis[131]. To establish the metabolomic changes that accompany this liver injury, the metabolites with significant differences in 
abundance between the HFD group and the LFD group were determined (Table 7). Eight metabolites were increased and one metabolite was decreased in the HFD group relative to the LFD group. As would be expected when comparing mice fed a diet high in fat to mice fed a low fat diet, two of the increased metabolites were fatty acids, whereas none of the decreased metabolite was fatty acids.

Table 8 shows the effects of arsenic in mice fed the low fat diet (LFD+As vs. LFD). There were no signs of liver injury in either of these groups, but there were significant metabolic differences resulting from arsenic exposure. The abundance of one metabolite was decreased while seven metabolites were increased in the LFD+As group compared to the LFD group. Three fatty acids were detected with significant regulation changes by arsenic. The levels of two fatty acids were increased while one was decreased.

Table 9 presents the results of arguably the most important comparison in this study: the metabolic differences between the HFD and HFD+As groups. Whereas the HFD produces mild liver injury in the form of steatosis, co-exposure to arsenic results in even more severe liver injury, with elevated markers of inflammation and cell death[131]. The combination of arsenic and a high fat diet (HFD+As) resulted in abundance changes in nine metabolites relative to the effect of a high fat diet alone (HFD), with six metabolites decreasing and three metabolites increasing. The metabolites that were lower in the HFD+As group were short-chain fatty acids, medium-chain fatty acids, the two acidic amino acids, aspartate and glutamate, and the anti-inflammatory amino acid glycine. The metabolites that were higher in the HFD+As group were the amino acids cysteine and lysine, and the citric acid cycle intermediate, citric acid 
Table 7. Metabolites with significance abundance difference between sample groups HFD and LFD.

\begin{tabular}{|c|c|c|c|c|c|}
\hline Name & $p$-value & ${ }^{l} t_{R}(\mathrm{~s})$ & ${ }^{2} t_{R}(\mathrm{~s})$ & CAS & $\begin{array}{l}\text { Fold } \\
\text { Change }^{a}\end{array}$ \\
\hline Tetradecanoic acid & $1.4 \times 10^{-2}$ & 2323.8 & 1.228 & $544-63-8$ & 1.5 \\
\hline Pentadecanoic acid & $1.4 \times 10^{-4}$ & 2432.6 & 1.243 & $1002-84-2$ & 2.3 \\
\hline L-Cysteine & $3.0 \times 10^{-5}$ & 2440.0 & 1.262 & $7048-04-6$ & 1.7 \\
\hline L-Threonine & $2.4 \times 10^{-2}$ & 1900.5 & 1.208 & $72-19-5$ & 2.1 \\
\hline L-Lysine & $5.2 \times 10^{-4}$ & 2625.7 & 1.272 & $56-87-1$ & -2.5 \\
\hline $\begin{array}{l}\text { Pentanoic acid, } \\
\text { 3-methyl-2-oxo- }\end{array}$ & $3.6 \times 10^{-2}$ & 2205.1 & 1.363 & $6628-79-1$ & 1.2 \\
\hline L-Methionine & $1.3 \times 10^{-2}$ & 2177.5 & 1.280 & $63-68-3$ & 1.4 \\
\hline L-Glutamic acid & $2.4 \times 10^{-2}$ & 2510.0 & 1.244 & $56-86-0$ & 1.5 \\
\hline Taurine & $3.8 \times 10^{-6}$ & 2117.5 & 1.351 & $107-35-7$ & 4.5 \\
\hline
\end{tabular}

a The sample group LFD is the reference group. "+" sign refers to abundance increase in sample group HFD, while “ _" sign refers to abundance decrease in HFD group.

${ }^{\mathrm{b}}$ Tentative identification without technical verification using authentic standards. 
Table 8. Metabolites with significance abundance difference between sample groups LFD+As and LFD.

\begin{tabular}{|c|c|c|c|c|c|}
\hline Name & $p$-value & ${ }^{l} t_{R}(\mathrm{~s})$ & ${ }^{2} t_{R}(\mathrm{~s})$ & CAS & $\begin{array}{l}\text { Fold } \\
\text { Change }^{a}\end{array}$ \\
\hline Heptanoic acid & $4.7 \times 10^{-3}$ & 1397.5 & 1.158 & $111-14-8$ & -1.4 \\
\hline Dodecanoic acid & $3.5 \times 10^{-3}$ & 2089.3 & 1.204 & $143-07-7$ & 1.7 \\
\hline Pentadecanoic acid & $3.3 \times 10^{-2}$ & 2432.6 & 1.243 & $1002-84-2$ & 1.3 \\
\hline L-Proline & $1.2 \times 10^{-2}$ & 1935.1 & 1.235 & $147-85-3$ & 1.2 \\
\hline L-Cysteine & $3.0 \times 10^{-2}$ & 2440.0 & 1.262 & $7048-04-6$ & 1.3 \\
\hline L-Methionine & $3.0 \times 10^{-3}$ & 2177.5 & 1.280 & $63-68-3$ & 1.4 \\
\hline 4-Aminobutanoic acid ${ }^{\mathbf{b}}$ & $8.4 \times 10^{-3}$ & 1926.5 & 1.188 & $56-12-2$ & 1.5 \\
\hline Pentanoic acid 3-methyl-2-oxo- ${ }^{\mathbf{b}}$ & $2.3 \times 10^{-3}$ & 2205.1 & 1.363 & $6628-79-1$ & 1.8 \\
\hline
\end{tabular}

a The sample group LFD is the reference group. "+" sign refers to abundance increase in sample group LFD+As, while “ _" sign refers to abundance decrease in LFD+As group.

${ }^{\mathrm{b}}$ Tentative identification without technical verification using authentic standards. 
Table 9. Metabolites with significance abundance difference between sample groups

$$
\text { HFD+As and HFD. }
$$

\begin{tabular}{|c|c|c|c|c|c|}
\hline Name & $p$-value & ${ }^{l} t_{R}(\mathrm{~s})$ & ${ }^{2} t_{R}(\mathrm{~s})$ & CAS & $\begin{array}{l}\text { Fold } \\
\text { Change } \\
a\end{array}$ \\
\hline Dodecanoic acid & $4.8 \times 10^{-2}$ & 2089.3 & 1.204 & $143-07-7$ & -1.3 \\
\hline Glycine & $5.0 \times 10^{-3}$ & 1655.0 & 1.159 & $56-40-6$ & -1.7 \\
\hline$\alpha$-Aminoisobutyric acid ${ }^{\mathrm{b}}$ & $3.4 \times 10^{-2}$ & 1686.9 & 1.166 & $594-61-6$ & -1.6 \\
\hline L-Glutamic acid & $4.2 \times 10^{-3}$ & 2510.0 & 1.244 & $56-86-0$ & -1.4 \\
\hline 2-Hydroxybutyric acid & $4.9 \times 10^{-2}$ & 1642.4 & 1.135 & $600-15-7$ & -1.4 \\
\hline L-Aspartic acid & $4.6 \times 10^{-2}$ & 2385.0 & 1.225 & $56-84-8$ & -1.2 \\
\hline L-Cysteine & $1.5 \times 10^{-2}$ & 2440.0 & 1.262 & $7048-04-6$ & 1.4 \\
\hline${\text { Citric } \text { acid }^{\mathbf{b}}}$ & $3.3 \times 10^{-2}$ & 2911.1 & 1.593 & $77-92-9$ & 1.7 \\
\hline L-Lysine & $6.0 \times 10^{-2}$ & 2625.7 & 1.272 & $56-87-1$ & 1.7 \\
\hline
\end{tabular}

a The sample group HFD is the reference group. "+" sign refers to abundance increase in sample group HFD+As, while “_" sign refers to abundance decrease in HFD+As group.

${ }^{\mathrm{b}}$ Tentative identification without technical verification using authentic standards.

\subsubsection{Analysis of Spike-in Samples}

A total of 30 samples were analyzed on GC $\times$ GC-TOF MS. Manual review of the peak lists generated by ChromaTOF shows that the 28 spiked-in metabolites were correctly identified in every sample. Of the 28 spiked-in metabolites, 23 metabolites were originally present in mouse liver extract. Therefore, the concentrations of these 23 metabolites are different from the other 5 spiked-in metabolites in each sample group. About 300 peak entries in each sample were assigned to a metabolite name via mass spectral matching. For retention index matching, the confidence interval of the empirical 
distribution function of the absolute retention index deviation to its mean value was set as $\mathrm{p} \leq 0.001 .20 .7 \%$ of the identified metabolites were confirmed by the first dimension retention index matching and $53.5 \%$ were also preserved due to the lack of retention index information in the NIST11 retention index database. However, $25.8 \%$ of the mass spectral matched metabolites were removed due to the large first dimension retention index deviation from the database values. The mean absolute deviation between the database value and experimental retention index of the removed metabolites is 308 index units (i.u.) with a standard deviation of 257 i.u. The corresponding values of the preserved metabolites are $28 \pm 12$ i.u. All 28 spiked-in metabolites are preserved in each of the 30 samples after the retention index matching. Metabolites butyric acid and L-tryptophan were preserved due to the lack of retention index values in the NIST11 database while the others were preserved by correct retention index match.

About 200-400 peaks were left in each sample for alignment. Peak picking is likely the primary cause for such variation. By manual validation, all 28 spiked-in metabolites were present in all 30 samples with a relative standard deviation (RSD) of the first and second dimensional retention times of $0.07 \%$ and $0.55 \%$, respectively, demonstrating a very good stability of the instrument during the two-dimensional GC separation and a high accuracy of ChromaTOF in determining peak location in the two-dimensional gas chromatograms.

Figure 17 depicts the peak area distribution of the 28 spiked-in metabolites in G10 before and after peak area conversion. A large variation in each box represents the peak area variation of the same compound between the 6 samples. By design, each compound should have the identical peak area among the 6 samples. However, ChromaTOF reports 

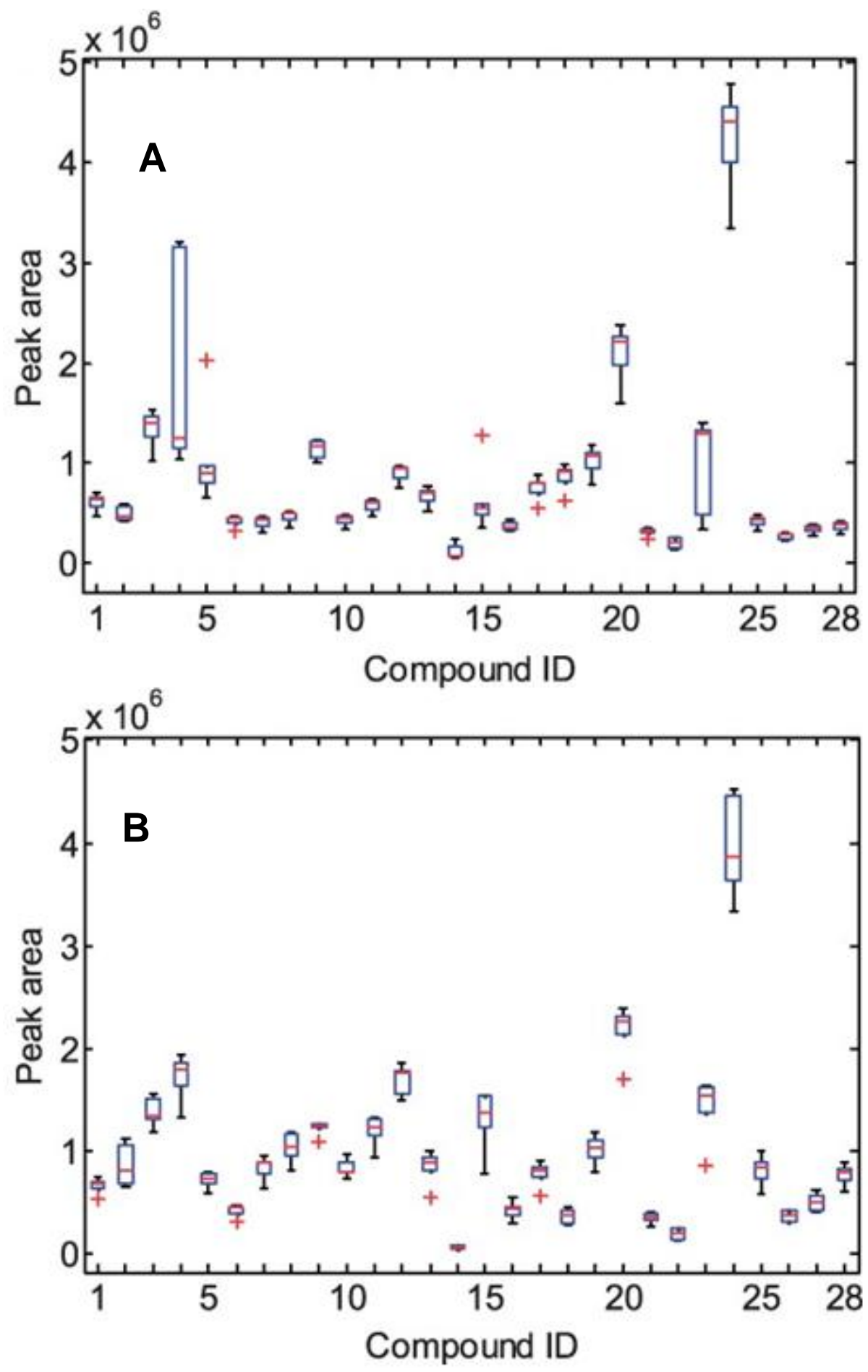

Figure 17. Boxplot of peak area of the 28 spiked-in metabolites in G10 before reference spectrum-based peak area conversion (A) and after the reference spectrum-based peak area conversion (B). + refers to the values of peak area considered as outliers. 
only the peak area of a quant mass (a fragment ion that has the highest quality for quantification), and it often chooses different quant mass for the same metabolite, depending on the data. Of the 28 metabolites, 10 metabolites in at least one of the 6 samples have quant mass different from the other samples. For instance, compound 4 (oxalic acid) in Figure 17(A) has 2 quant mass values and the value of peak area has a large deviation, ranging from $1,041,108$ to $3,206,235$. After reference spectrum-based peak area conversion, the span of peak area is greatly reduced to $1,329,043-1,935,698$ (Figure 17(B)), demonstrating the effectiveness of the reference spectrum-based peak area conversion. The converted peak area values not only reduced the variation in peak area, but also guarantee the consistency of the values of peak area across samples for the downstream statistical analysis.

To demonstrate whether the concentration differences of the spiked-in metabolite standards can be recognized from the alignment table, a two-tailed t-test was used to check the mean difference of the peak area of each compound between sample groups through different p-value settings. The true positive rate (TPR) and the false positive rate (FPR) are used as measures for metabolite relative quantification. Figure 18 depicts the ROC curve of recognizing the concentration difference of the spiked-in metabolite standards between sample groups using the alignment results. As expected, the FPR increases with the increase of TPR. The TPR levels off at 1.0 when the FPR reaches 0.9 between all the comparing sample groups. The AUC of the ROC curve of G10 vs. G20 is 


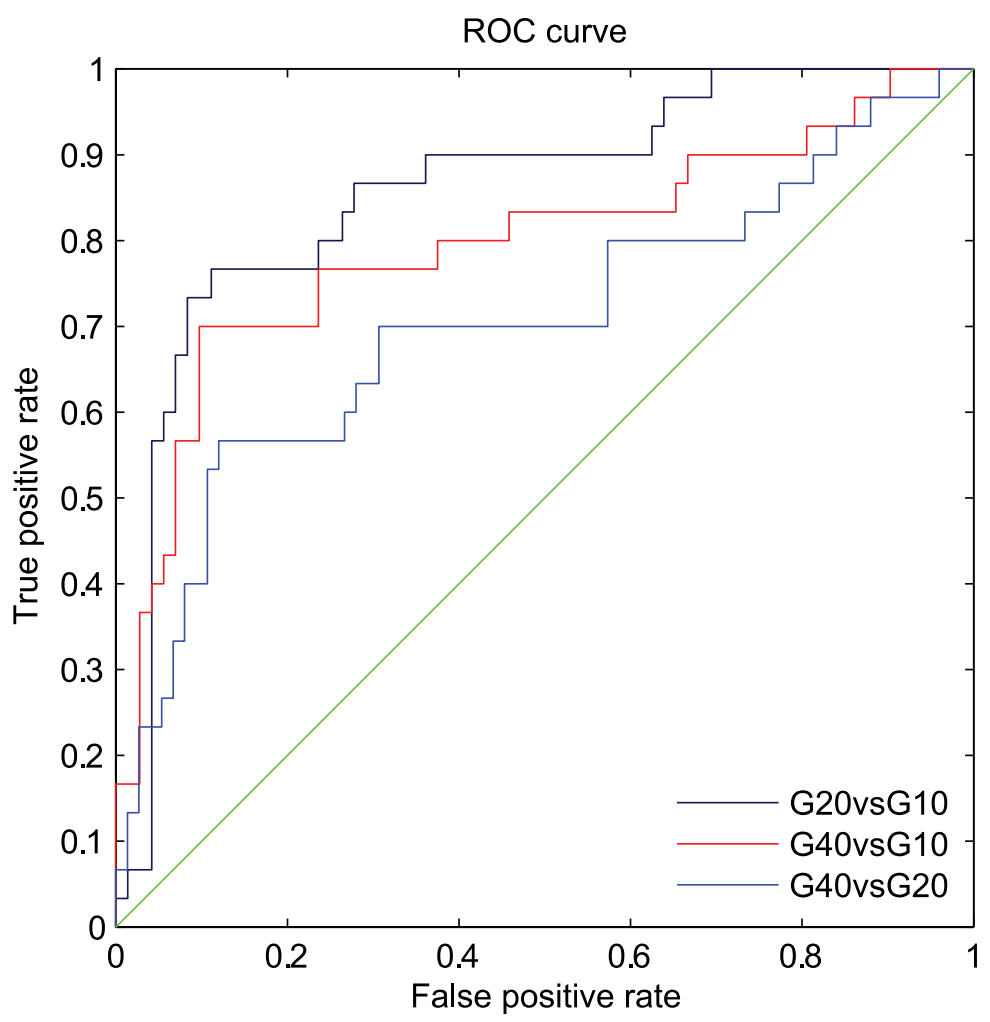

Figure 18. The receiver operating characteristic (ROC) curve of the spike-in data. The area under curve (AUC) of the three comparisons is $0.87,0.71$, and 0.81 for G20 vs. G10, G40 vs. G10 and G40 vs. G20, respectively.

0.87, while the AUC of the ROC curve of G20 vs. G40 and G10 vs. G40 is 0.71 and 0.81 , respectively. A high value of AUC indicates a high accuracy of recognizing the concentration difference of the spiked-in compounds between sample groups, which is achieved on the basis of correct alignment of the spiked-in compounds. Moreover, it is worth mentioning that the spiked-in metabolites that were not recognized as metabolites with significant concentration difference between the two testing sample groups are all present in the mouse liver extract before the addition of the mixture of authentic standards. This means that the concentration differences of these metabolites between 
sample groups may be much smaller than $2: 1$ or $4: 1$, depending on the amount of the metabolites in each sample of mouse liver extract.

\subsection{Discussion}

Multiple data analysis steps were involved in analysis of the $\mathrm{GC} \times \mathrm{GC}-\mathrm{TOF}$ MS data in this study. In order to control the false discovery rate, the experimental data were analyzed using different values of FDR threshold including $q \leq 0.05,0.1,0.15,0.2$ and

0.3. The numbers of metabolites detected with significant abundance change between two sample groups are listed in Table 10. It is generally known that the statistical power increases as the FDR increases at a fixed sample size, while the power increases as the sample size increases at a fixed FDR[145]. Consequently, a larger FDR threshold is required to achieve a desirable power when the sample size is small. For this reason, we selected $\mathrm{q} \leq 0.2$ to achieve a reasonable power due to the small sample size in this study. Figure 19 depicts the clustering results of the four sample groups. The results presented here demonstrate that both high dietary fat intake and environmental arsenic exposure exert effects on liver metabolism, and that the combination of the two exposures yields a unique metabolite profile that may offer some insight into the mechanisms of liver injury. Previously, we showed that mice fed the so-called Western diet (a diet high in milk fat and cholesterol) for 10 weeks gained more weight and had significantly more fat in their livers than mice fed a low fat $\operatorname{diet}[131]$, consistent with other studies using this $\operatorname{diet}[146,147]$. Importantly, co-exposure to arsenic-containing drinking water resulted in significantly greater inflammatory liver injury in this model[131], suggesting that arsenic may be a second hit that promotes progression from steatosis to steatohepatitis[127]. 


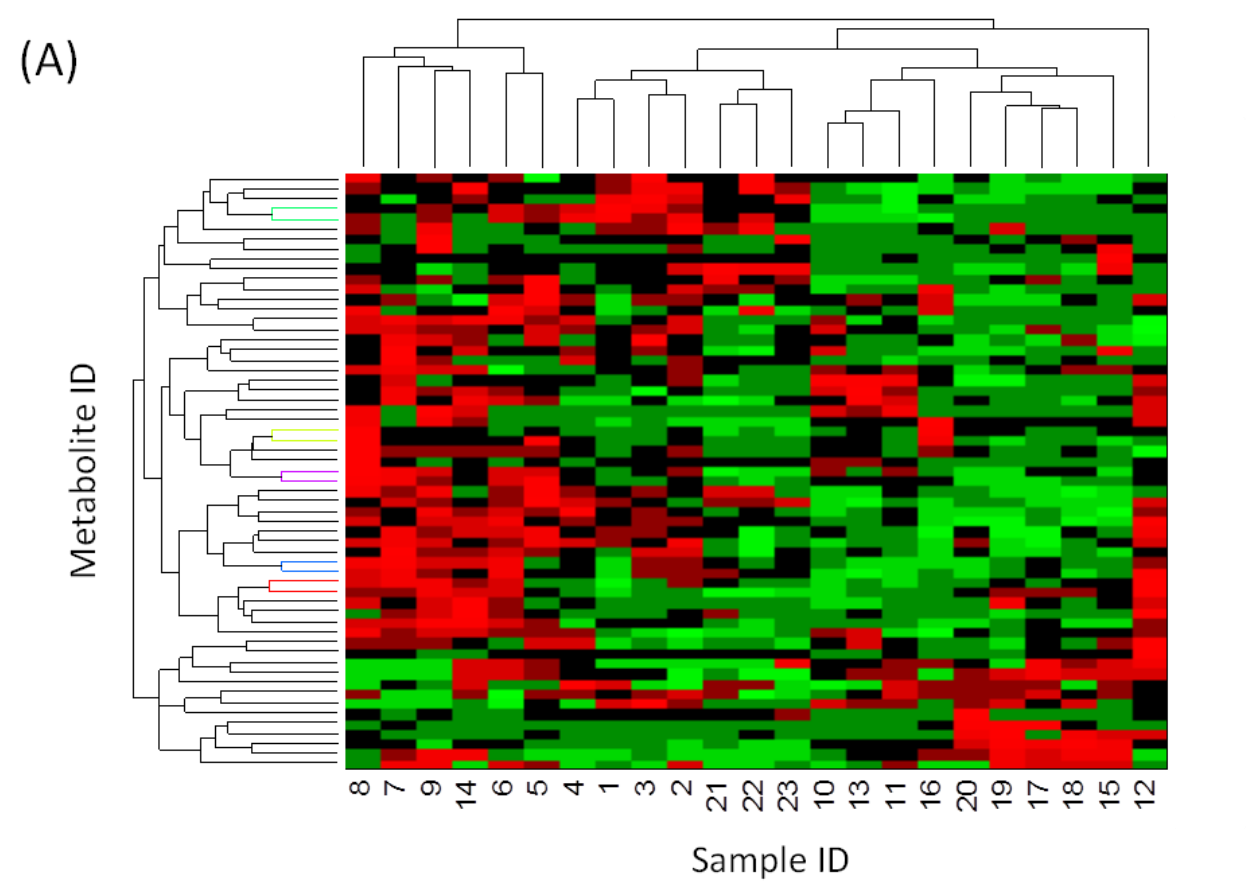

(B)

\begin{tabular}{|l|l|l|l|l|l|}
\hline $\begin{array}{l}\text { Sample } \\
\text { Groups }\end{array}$ & $\begin{array}{l}\text { HFD vs. } \\
\text { LFD }\end{array}$ & $\begin{array}{l}\text { HFD+As } \\
\text { vs. HFD }\end{array}$ & $\begin{array}{l}\text { HFD+As } \\
\text { vs. LFD }\end{array}$ & $\begin{array}{l}\text { HFD+As } \\
\text { vs. LFD+As }\end{array}$ & $\begin{array}{l}\text { LFD+As } \\
\text { vs. LFD }\end{array}$ \\
\hline Accuracy & 0.909 & 1.00 & 0.833 & 0.667 & 0.833 \\
\hline
\end{tabular}

Figure 19. Clustering analysis of the four sample groups using fuzzy $\mathrm{C}$ mean algorithm.

The input data were the abundance of all metabolites that were detected with significant regulation changes between two or more sample groups when the FDR threshold $\mathrm{q} \leqslant 0.2$. (A) depicts the clustering results of all four sample groups. The overall clustering accuracy reached 0.70. (B) displays the clustering accuracy between two sample groups. 
Table 10. The number of metabolites with significant differences between sample groups with different values of FDR threshold. It should be noted that the number of metabolites detected with significant difference between sample groups are different from these listed in Tables $7,8,9$. The number of metabolites listed in this table was directly calculated from the alignment table. That is the identifications of these compounds were not yet confirmed using the methods described in section 4.3.1.

\begin{tabular}{|c|c|c|c|c|}
\hline Sample Groups & $\begin{array}{c}\text { HFD+As } \\
\text { vs. HFD }\end{array}$ & $\begin{array}{c}\text { HFD vs. } \\
\text { LFD }\end{array}$ & $\begin{array}{c}\text { LFD+As } \\
\text { vs. LFD }\end{array}$ & $\begin{array}{c}\text { HFD+As vs. } \\
\text { LFD+As }\end{array}$ \\
\hline$q \leq 0.05$ & 9 & 8 & 0 & 25 \\
\hline$q \leq 0.1$ & 14 & 12 & 6 & 30 \\
\hline$q \leq 0.15$ & 15 & 20 & 10 & 31 \\
\hline$q \leq 0.2$ & 25 & 20 & 12 & 36 \\
\hline$q \leq 0.3$ & 34 & 30 & 16 & 39 \\
\hline
\end{tabular}


In the context of the two hit hypothesis of steatohepatitis[127], one should consider how the metabolic changes elicited by the first hit (high fat diet) are affected by the second hit (arsenic). The high fat diet significantly altered nine metabolites (Table 7), and the addition of arsenic resulted in further alterations in three of these metabolites (Table 9). The HFD-induced increase in cysteine was amplified, whereas the increase in glutamate was blocked, by co-exposure to arsenic. Also, the HFD-induced decrease in lysine was reversed by arsenic. Furthermore, six additional metabolites were changed by the combination of the high fat diet and arsenic that were not altered by the high fat diet alone. Among these, glycine was decreased by the combination of arsenic and a high fat diet. Therefore, all three of the amino acids that constitute the antioxidant glutathione (glutamate, cysteine and glycine) were altered, with glutamate and glycine levels lower and cysteine levels higher. Under normal conditions, cysteine concentrations are near the $\mathrm{K}_{\mathrm{m}}$ for the rate-limiting enzyme glutathione synthesis[148], and an increase in cysteine would be expected to increase the rate of synthesis. Indeed, Literature reported that mice exposed to arsenic for 8 weeks had elevated levels of hepatic glutathione[149]. Thus, arsenic exposure may evoke a compensatory increase in glutathione synthesis in the liver, at least in response to these relatively short exposures. The increase in cysteine observed in the current study could reflect increased uptake of this rate-limiting amino acid in an attempt by the liver to compensate for an increased demand for glutathione synthesis. Cysteine is taken up by the cystine/glutamate antiporter, which exchanges extracellular cystine for intracellular gluatamate. The expression of this transporter is induced by oxidants, electrophiles and inflammatory mediators. Therefore, the increase in cysteine 
and decrease in glutamate observed in the present study may reflect increased activity of this transporter.

In addition to altered glycine, alterations in five other metabolites were specific to the HFD+As group. Three of these were fatty acids or fatty acid metabolites, and each of these was found to be decreased relative to the high fat diet alone. The high fat diet used in this study contained $21 \%(\mathrm{w} / \mathrm{w})$ milk fat. The major fatty acids found in milk fat are palmitic acid (C16:0, 30\% of the fatty acids), stearic acid (C18:0, 12\%), myristic acid $(\mathrm{C} 14: 0,11 \%)$ and lauric acid $(\mathrm{C} 12: 0,3.3 \%)$ [150]. We observed an increase in myristic acid in the livers of mice fed a high milk fat diet when compared to the mice fed the low fat control diet, but not palmitic, stearic or lauric acids.

Interestingly, when mice were co-exposed to arsenic and a high fat diet, there was a decline in hepatic lauric acid levels. Diets high in saturated medium chain fatty acids, such as lauric acid, protect against inflammatory liver injury caused by chronic alcohol ingestion[151] or acute endotoxin administration[152]. Recently, in a model of non-alcoholic fatty liver disease where rats were fed diets supplying $70 \%$ of calories from fat, replacing fat from corn oil with fats enriched in medium-chain triglycerides decreased steatosis and liver injury[153]. The mechanisms by which medium-chain lipids in general and lauric acid in particular inhibit inflammatory liver injury are not clear. In fact, some in vitro studies have found that lauric acid can by pro-inflammatory. For example, lauric acid increased inflammatory cytokine production by astrocytes and macrophages via activation of TLR4 signaling[154,155], but it had no effect on cytokine production by adipocytes or endothelial cells[156,157]. It is possible that hepatic lauric acid contributes to inflammatory liver injury independently of any effects on cytokine 
production; its levels may influence responses to cytokines, or it may be a marker of alterations in other critical pathways like mitochondrial fatty acid oxidation[158]. Additional studies will be needed to determine how arsenic and a high fat diet contribute to the down-regulation of lauric acid, and whether this down-regulation contributes to increased inflammation and/or injury in this model.

Two other fatty acid metabolites were decreased in the livers of mice fed arsenic and a high fat diet: 2-hydroxybutyrate and 2-aminobutyrate. Both of these compounds can be produced by the catabolism of threonine, and 2-hydroxybutyrate can also be formed in the conversion of methionine to cysteine in the transsulfuration pathway. A decrease in serum 2-hydroxybutyrate was recently reported in children with cystic fibrosis[159], a disease associated with increased inflammation. It remains to be determined whether decreases in these metabolites are common to early stages of inflammatory tissue injury. The decrease in glycine in the HFD+As group relative to the HFD group may be an important clue as to the mechanism by which the combination of arsenic and a high fat diet leads to inflammatory liver injury. Glycine supplementation protects the liver in a number of inflammatory injury models[160,161] by acting on glycine-gated chloride channels on Kupffer cells to inhibit inflammatory cytokine production[160,162,163]. The glycine deficiency in our model may have promoted pro-inflammatory signaling. In support of this, we observed a dramatic increase in the number of activated Kupffer cells, as determined by F4/80 staining, in response to arsenic exposure in the HFD group[131]. It is unclear how arsenic and a high fat diet deplete hepatic glycine, but altered glycine metabolism could play a role. Threonine dehydratase is inhibited by cysteine[164], which is elevated by arsenic and a high fat diet, both alone and in combination. Serine 
hydroxymethyltransferase has an active site cysteine as well as two closely-spaced (vicinal) surface-exposed cysteines that can alter activity when modified by sulfhydryl reagents[165, 166]. Arsenic binds to vicinal dithiols[167], but it is not known whether serine hydroxymethyltransferase is a target of arsenic binding.

Metabolomics is a powerful approach for the discovery of pathways altered by environmental and dietary exposures related to disease. When used to investigate the combined effects of a high fat diet and arsenic in producing fatty liver with inflammation and cell death, metabolomic analyses revealed significant changes in the metabolism of short-chain and medium-chain fatty acids, as well as alterations in the pathways producing the anti-inflammatory amino acid glycine. Further investigations into the mechanisms by which these pathways are altered and the specific roles they play in the progression of non-alcoholic fatty liver disease are warranted.

Metabolites in mouse liver can have very diverse chemical characteristics. In this work, metabolites were extracted by water and methanol. Most of the nonpolar metabolites were lost during this analytical step. The extracted metabolites were then analyzed on GC $\times$ GC-TOF MS. Even though the GC $\times$ GC-TOF MS has a large separation power, it may not be enough to resolve all metabolites, resulting overlapping chromatographic peaks that introduces significant challenges for metabolite identification and quantification. In this work, metabolite identification was first achieved by matching the experimental mass spectra to the mass spectra recorded in the NIST mass spectral library. The incompleteness of the existing mass spectral library does not only introduce a certain degree of false-positive identifications, but also leaves a number of chromatographic peaks without any compound identification. All of these technical limitations in the current study prevent 
us from seeing the entire picture of the metabolite profile in mouse liver. Further studies such as using different extraction methods, combining both GC $\times$ GC-TOF MS and 2D LC-MS, and applying multiple mass spectral libraries for metabolite identification may provide more comprehensive results. Further studies with a larger sample size will be also necessary to confirm the results in this study.

The high accuracy of clustering between sample groups HFD vs. LFD, HFD+As vs. HFD and LFD+As vs. LFD indicates that the high-fat diet and arsenic contaminated water can significantly change the metabolite profile in mouse liver. Comparing the clustering accuracy of (A) and (B), the low value of overall clustering accuracy for clustering all four sample groups in (A) was mainly caused by a ceartin degree of similarity of metabolite profile between sample groups LFD+As and HFD+As.

\subsection{Conclusions}

GC $\times$ GC-TOF MS was used to identify metabolites that were significantly increased or decreased in the livers of mice fed a Western diet (a diet high in fat and cholesterol) and co-exposed to arsenic-contaminated drinking water, to investigate potential mechanisms of this diet-environment interaction. The results showed that there are distinct hepatic metabolomic profiles associated with eating a high fat diet, drinking arsenic contaminated water, and the combination of the two. Among the metabolites that were decreased when arsenic exposure was combined with a high fat diet were hepatoprotective short chain and medium-chain fatty acid metabolites and the anti-inflammatory amino acid glycine. These results are consistent with the observed increase in inflammation and cell death in the livers of these mice and point to potentially novel mechanisms by which these 
metabolic pathways could be altered by arsenic in the context of diet-induced fatty liver disease. 


\section{CHAPTER 5}

\section{HEPATIC AND FECAL METABOLOMIC ANALYSIS OF THE EFFECTS OF LACTOBACILLUS RHAMNOSUS GG ON ALCOHOLIC FATTY LIVER DISEASE IN MICE}

\subsection{Introduction}

Lactobacillus rhamnosus GG (LGG), a strain of the bacterium, Lactobacillus rhamnosus, has a great affinity for human intestinal mucosal cells. Previous studies in our laboratory and others demonstrated that LGG is effective in the treatment of alcohol-induced liver injury in rodents[168-171]. More recently, we have shown that LGG culture supernatant (LGGs) is effective in prevention of acute and chronic alcohol exposure-induced fatty liver[172,173]. LGGs administration markedly attenuates the effects of alcohol exposure on intestinal barrier dysfunction, by increasing intestinal mucus factors and epithelial tight junction protein expression, leading to decreased circulation endotoxin levels. In addition, LGGs activates hepatic 5' adenosine monophosphate-activated protein kinase- $\alpha$ (AMPK $\alpha$ ) phosphorylation, which is critical for liver lipid degradation[174].

To gain insight into the metabolic mechanisms by which LGGs exerts an influence in alcoholic liver disease (ALD), investigation of metabolome alteration using high throughput metabolomics analysis is needed. Comprehensive two-dimensional gas chromatography time-of-flight mass spectrometry ( $\mathrm{GC} \times \mathrm{GC}-\mathrm{TOF} \mathrm{MS})$ is a powerful analytical platform in metabolomics. It uses two distinctly different capillary GC columns 
of different polarities connected via a thermal modulator[137,138,175]. The analytes co-eluted from the first column are further separated in the second column because of the difference of column temperature and the chromatographic polarities. The further separated analytes are detected by a time-of-flight mass spectrometer. The $\mathrm{GC} \times \mathrm{GC}-\mathrm{TOF}$ MS platform has significant advantages for complex sample analysis including: an order-of-magnitude increase in separation capacity, significant increase in signal-to-noise ratio and dynamic range and improvement of mass spectral deconvolution and similarity matches[176-178].

The objectives of this study were to determine if the beneficial effects of LGGs administration on the prevention of ALD are associated with alterations in metabolic profiles in the liver and fecal metabolome. Male C57BL/6 mice were pair-fed either an isocaloric control diet (PF) or alcohol-containing diet (AF) for 4 weeks with or without LGGs co-exposure. Liver and fecal samples were collected at the end of the experiments. The metabolite extracts from mouse livers and feces were analyzed on GC×GC-TOF MS for metabolic profiling.

\subsection{Experimental Methods}

\subsubsection{Animals and Diets}

C57BL/6 male mice obtained from Harlan (Indianapolis, IN) were fed a modified Lieber-DeCarli liquid diet (Research Diet, New Brunswick, NJ). Mice were fed EtOH-containing diets (35\% of total calories, AF) ad libitum for 4 weeks. Control mice were pair-fed isocaloric diet in which EtOH was replaced with maltose-dextrin. LGG was cultured following the instruction provided by ATCC (Manasses, VA). The culture 
supernatant was collected when LGG bacterial growth reached $10^{9} \mathrm{CFU} / \mathrm{ml}$. The LGG supernatant (LGGs) was added to the liquid diet at a dose equivalent to $10^{9}$ $\mathrm{CFU} / \mathrm{mouse} / \mathrm{day}$. At the end of the experiment, the mice were anesthetized, and the fecal samples and liver samples were harvested. A portion of liver samples was fixed in formalin, while the rest of the liver samples and the fecal samples were frozen immediately in liquid nitrogen and stored at $-80^{\circ} \mathrm{C}$ for later assays. The animal protocol was approved by the University of Louisville Institutional Animal Care and Use Committee.

\subsubsection{Liver Oil Red O Staining and Liver Total Free Fatty Acid Analysis}

Frozen liver sections were processed for staining with Oil red $\mathrm{O}$ and then studied by light microscopy. Hepatic free fatty acid levels were measured using Infinity kits (Thermo Scientific, Waltham, MA).

\subsubsection{Metabolite Sample Preparation}

Fecal sample was first weighed and ground on ice. Each grounded fecal sample was added ice cold $80 \%$ methanol with a ratio of $50 \mathrm{mg}$ feces in $1 \mathrm{~mL} 80 \%$ methanol. The mixture was vortexed for $10 \mathrm{~min}$ followed by centrifugation for $10 \mathrm{~min}$ at $16000 \mathrm{rpm}$. The supernatant $200 \mu \mathrm{L}$ was aspirated into a plastic tube and dried by SpeedVac overnight. The dried metabolite extracts were dissolved in $30 \mu \mathrm{L}$ ethoxyamine hydrochloride solution (30 $\mathrm{mg} / \mathrm{mL})$ and vigorously vortex-mixed for $1 \mathrm{~min}$. Ethoxymation was carried out at $70{ }^{\circ} \mathrm{C}$ for 1 hour. After adding $30 \mu \mathrm{L}$ $\mathrm{N}$-(tert-butyldimethylsilyl)-N methyltrifluoroacetamide (MTBSTFA) mixed with 1\% 
tert-Butyldimethylchlorosilane (TBDMSCI), derivatization was carried out at $70{ }^{\circ} \mathrm{C}$ for 1 hour.

To prepare liver samples, a sample of liver tissue was weighed and then homogenized for $2 \mathrm{~min}$ after adding water at a ratio of $100 \mathrm{mg}$ liver tissue/mL water. The homogenized sample was then stored at $-80{ }^{\circ} \mathrm{C}$ until use. A $100 \mu \mathrm{L}$ aliquot of the homogenized liver sample and $400 \mu \mathrm{L}$ methanol were mixed and vortexed for 1 min followed by centrifugation at room temperature for $10 \mathrm{~min}$ at $15000 \mathrm{rpm} .400 \mu \mathrm{L}$ of the supernatant was aspirated into a plastic tube and dried by $\mathrm{N} 2$ flow. The metabolites extracts were then dissolved in $40 \mu \mathrm{L}$ ethoxyamine hydrochloride solution $(30 \mathrm{mg} / \mathrm{mL})$ and vigorously vortex-mixed for $1 \mathrm{~min}$. Ethoxymation and derivatization were achieved in the same way as for the fecal samples.

In order to verify the compound identification, a total of 61 compound standards were prepared in equal molarity and analyzed on $\mathrm{GC} \times \mathrm{GC}-\mathrm{TOF}$ MS. The ethoxymation and derivatization of the fecal sample, liver sample, and compound standards were carried out right before $\mathrm{GC} \times \mathrm{GC}-\mathrm{TOF} \mathrm{MS}$ analysis, respectively.

\subsection{4. $\mathrm{GC} \times \mathrm{GC}-\mathrm{TOF} \mathrm{MS}$ Analysis}

The LECO Pegasus 4D GC×GC-TOF MS instrument was equipped with an Agilent 6890 gas chromatograph and a Gerstel MPS2 auto-sampler (GERSTEL Inc., Linthicum, MD), featuring a LECO two-stage cryogenic modulator and secondary oven. The primary column was a $60 \mathrm{~m} \times 0.25 \mathrm{~mm}^{1} d_{c} \times 0.25 \mu \mathrm{m}^{1} d_{f}$, DB-5ms GC capillary column (phenyl arylene polymer virtually equivalent to a (5\%-phenyl)-methylpolysiloxane). A second GC column of $1 \mathrm{~m} \times 0.25 \quad \mathrm{~mm} \quad{ }^{1} d_{c} \times 0.25 \quad \mu \mathrm{m} \quad{ }^{2} d_{f}, \quad$ DB17ms ((50\%-phenyl)-methylpolysiloxane) was placed inside the secondary GC oven after the 
thermal modulator. Both columns were obtained from Agilent Technologies (Agilent Technologies J\&W, Santa Clara, CA). The helium carrier gas (99.999\% purity) flow rate was set to $2.0 \mathrm{~mL} / \mathrm{min}$ at a corrected constant flow via pressure ramps. The inlet temperature was set at $280{ }^{\circ} \mathrm{C}$. The primary column temperature was programmed with an initial temperature of $60{ }^{\circ} \mathrm{C}$ for $0.5 \mathrm{~min}$ and then ramped at $5{ }^{\circ} \mathrm{C} / \mathrm{min}$ to $280{ }^{\circ} \mathrm{C}$ and kept for $15 \mathrm{~min}$. The secondary column temperature program was set to an initial temperature of $70{ }^{\circ} \mathrm{C}$ for $0.5 \mathrm{~min}$ and then also ramped at the same temperature gradient employed in the first column to $280{ }^{\circ} \mathrm{C}$ accordingly. The thermal modulator was set to $+15{ }^{\circ} \mathrm{C}$ relative to the secondary oven, and a modulation time of $P_{M}=2 \mathrm{~s}$ was used. The mass range was set as $29-800 \mathrm{~m} / \mathrm{z}$ with an acquisition rate of 200 mass spectra per second. The ion source chamber was set at $230{ }^{\circ} \mathrm{C}$ with the transfer line temperature set to $280{ }^{\circ} \mathrm{C}$, and the detector voltage was $1450 \mathrm{~V}$ with electron energy of $70 \mathrm{eV}$. The acceleration voltage was turned on after a solvent delay of $675 \mathrm{~s}$. The split ratio was set at 40:1 for liver samples and 10:1 for fecal samples.

\subsubsection{Data Analysis}

The GC $\times$ GC-TOF MS data were processed using LECO's instrument control software ChromaTOF for peak picking and tentative metabolite identification, followed by peak merging, retention index filtering, peak list alignment, normalization, and statistical significance test using MetPP[141]. Two-way ANOVA test with a threshold of false discovery rate $p<0.05$ was used to determine whether a metabolite has a significance difference of abundance level between sample groups. 
For metabolite identification using ChromaTOF, each chromatographic peak was tentatively assigned to a metabolite if its experimental mass spectrum and a NIST11 MS library spectrum have a spectral similarity score higher than 600 (the maximum value of spectral similarity was defined as 1000 in ChromaTOF). If a chromatographic peak was tentatively assigned to a metabolite by mass spectral matching, the other top four ranked metabolite candidates were considered as the identification results of mass spectral matching for this chromatographic peak. The tentatively assigned metabolites were further filtered by retention index matching using iMatch software with a $p$-value threshold of $p<0.001[179]$. To further verify the identification of metabolites detected with significant abundance difference between sample groups, the authentic standards of these metabolites, if commercially available, were analyzed on GC×GC-TOF MS under

the same experimental conditions as the biological samples analyzed. A tentative metabolite assignment was considered as a correct identification only if the experimental information of the authentic metabolite agrees with the corresponding information of the chromatographic peak in the biological samples, i.e., difference of the first dimension retention time $\leq 10 \mathrm{~s}$, difference of the second dimension retention time $\leq 0.05 \mathrm{~s}$, and the mass spectral similarity $\geq 700$.

\subsection{Results}

Four biological sample groups were formed in this study, pair-fed control mice without LGGs supplementation (PF-0, $\mathrm{n}=5$ ); pair-fed control mice with exposure to LGGs (PF-1, $\mathrm{n}=7$ ); alcohol fed mice without LGGs supplementation (AF-0, $\mathrm{n}=4$ ); and alcohol fed mice with LGGs supplementation $(\mathrm{AF}-1, \mathrm{n}=6)$. Metabolite identification was done in three sequential steps as described in our previous work, including mass spectral 
matching, retention index matching, and comparison with authentic standards[16]. We compared the metabolic profile differences between PF- 0 and AF- $0, \mathrm{PF}-1$ and PF- 0 , and AF-1 and AF-0 in mouse liver and fecal samples, respectively.

GC $\times$ GC-TOF MS instrument data provide four pieces of information for each metabolite, the first dimension retention time ${ }^{l} t_{R}$, the second dimension retention ${ }^{2} t_{R}$, fragment ion $m / z$, and peak height of each fragment ion. The information of fragment ion $\mathrm{m} / \mathrm{z}$ and peak height forms the mass spectrum of the metabolite. Figure 20 is a contour plot of the GC $\times$ GC-TOF MS data acquired from a fecal sample randomly selected from sample group PF-0. Each chromatographic peak in Figure 20 is composed of many data points, where the number of data points in each chromatographic peak depends on the size of the chromatographic peak. The color of each data point is the signal intensity, i.e., the total ion current of all fragment ions in the corresponding mass spectrum. The inset in Figure 20 demonstrates the increased separation power of $\mathrm{GC} \times \mathrm{GC}-\mathrm{TOF} \mathrm{MS}$ system. The two chromatographic peaks have identical retention time in the first dimension GC, but are completely separated from each other on the second dimension GC. Therefore, these two chromatographic peaks will co-elute on a GC-MS system and generate a mixed mass spectrum, which may induce false identification and quantification. 


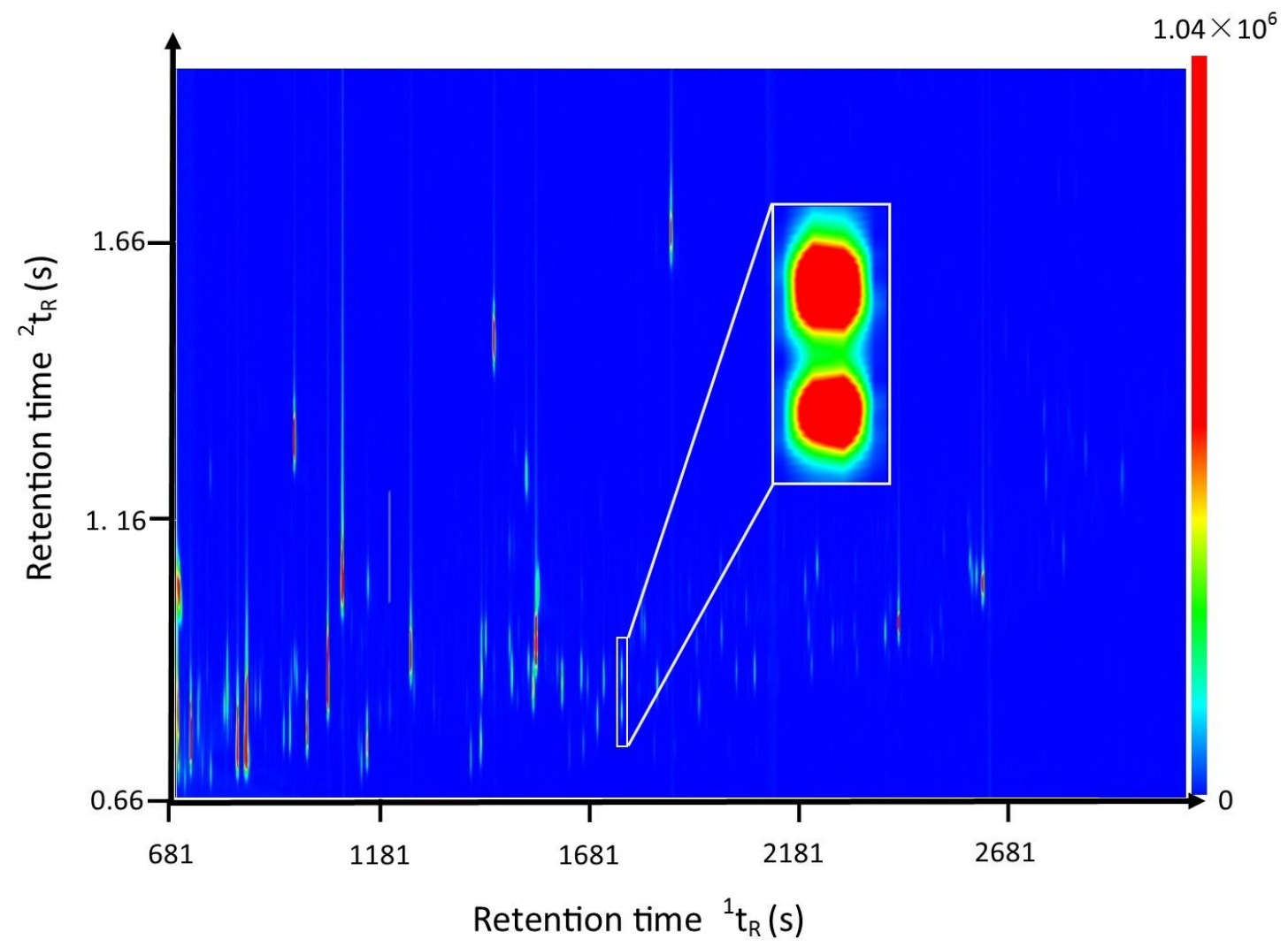

Figure 20. Sample GC $\times$ GC-TOF MS chromatogram of metabolite extract from mouse fecal samples. The $\mathrm{x}$-axis is the first dimension retention time ${ }^{1} t_{R}$ in seconds. The $y$-axis is the second dimension retention time ${ }^{2} t_{R}$ in seconds. The color bar shows the signal intensity of each peak plotted on the chromatogram in total ion current.

By setting spectral similarity threshold to $\geq 600$ in ChromaTOF, 440-1100 and 490-840 and metabolites were respectively identified from the liver and fecal samples. The numbers of metabolites detected in liver samples and fecal samples were further reduced to 390-880 and 435-727 after retention index matching. After cross sample alignment and removing chromatographic peaks detected in blank samples, 236 metabolites presented in 
more than $75 \%$ of liver samples in at least one sample group, while 212 in the fecal samples.

Two-way ANOVA tests were employed to recognize metabolite with significant abundance changes between sample groups contributed by alcohol, LGGs, or their interaction. A term fold-change was defined as the ratio of the large abundance value (peak area) of a metabolite in one group divided by the small abundance value of the same metabolite in the other group. The positive sign and negative sign indicate the abundance increase and decrease in the testing sample group compared to the reference sample group, respectively.

Figure $21 \mathrm{~A}$ is an example of the metabolite abundance level changes detected in the liver samples. Abundance distribution of the metabolite, hexanoic acid, in liver samples was detected in the four sample groups, PF-0, PF-1, AF-0 and AF-1. Hexanoic acid (caproic acid), a natural fatty acid existing in all mammals, has been shown to be beneficial in high density lipoprotein synthesis in the liver. Compared with the abundance level of this metabolite in control group PF-0, the level of this metabolite was increased with a fold change of 1.56 in group AF-0, and 1.09 fold decrease in group PF-1. Comparing its abundance levels between groups AF-1 and AF-0, this metabolite is decreased with a fold change of 1.23 in the AF-1 group. The $p$-values for the factors of alcohol, LGGs, and their interaction are $0.002,0.24$, and 0.80 , respectively. The alcohol factor has the smallest $p$-value of 0.002 , indicating that this metabolite (hexanoic acid) has a significant alteration in abundance level in mouse livers because of the alcohol factor, while LGGs alone $(p=0.24)$ and the interaction of LGGs and alcohol $(p=0.80)$ did not significantly change the abundance level of this metabolite in liver. 
Figure 21B depicts the abundance distribution of the same metabolite (hexanoic acid) in feces among the four sample groups. The trend of abundance distribution of this metabolite in fecal samples is completely different from that in the liver samples. Compared with the abundance of this metabolite in control group PF-0, the abundance level of this metabolite is decreased 1.54 fold in group PF-1 and by a1.25 fold decrease in sample group AF-0. Hexanoic acid is increased with a fold change of 1.58 in group AF-1 compared to AF-0. The $p$-values of alcohol, LGGs, and their interaction are $0.05,0.92$, and 0.003 , respectively. The large $p$-value of 0.92 indicates that LGGs alone did not significantly alter the abundance level of this metabolite in the mouse fecal metabolome, while alcohol alone affected the abundance level of this metabolite $(p=0.05)$. Importantly, the smallest magnitude of $p$-value $(p=0.003)$ for the interaction between alcohol and LGGs indicates that the abundance level of this metabolite was significantly changed owing to the synergistic effect of alcohol and LGGs on mouse fecal metabolome.

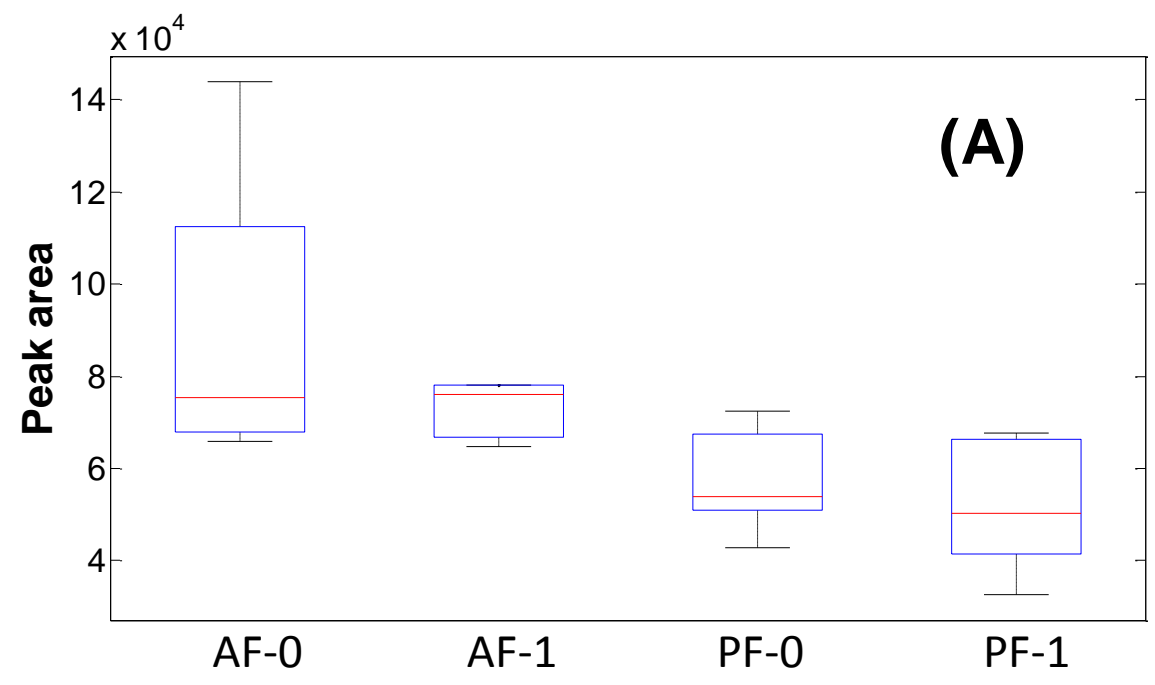




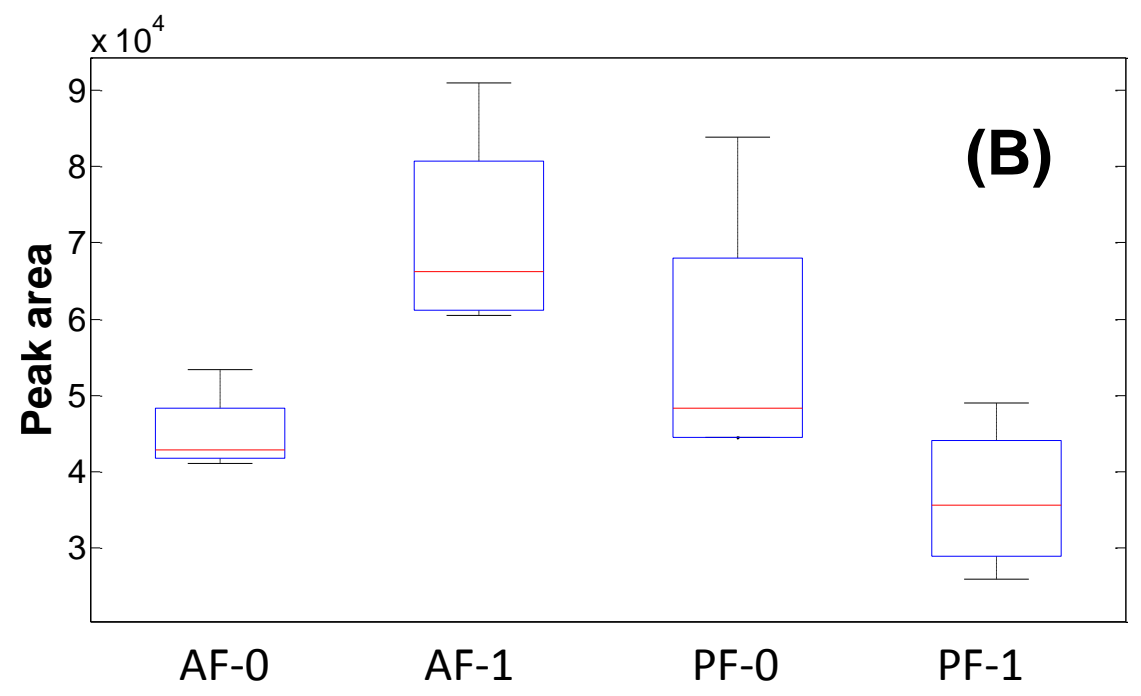

Figure 21. Abundance distribution of metabolite hexanoic acid in four sample groups. (A) is in liver samples, and (B) is in fecal samples.

Table 11 lists all metabolites that were detected with significant abundance changes from liver samples between the four sample groups with a threshold of $p \leq 0.05$. These compounds include eight free fatty acids, six amino acids, three other acids and three other small compounds. Among the eight fatty acids, one is short chain fatty acid (SCFA, acetic acid), two are medium chain fatty acids (MCFA, hexanoic acid and dodecanoic acid), one long chain fatty acid (LCFA, tetradecanoic acid), two monounsaturated fatty acids (trans-9-octadecenoic acid and cis-9-hexadecenoic acid) and two polyunsaturated fatty acids (mead acid and arachidonic acid). The abundance levels of all four saturated fatty acids and cis-9-hexadecenoic acid were increased when the mice were fed with alcohol (column FC-2 in Table 11). However, the abundance levels of these acids were all decreased when the alcohol fed mice were also exposed to LGGs (column FC-1 in 
Table 11). Figure 22 depicts the results of total free fatty acid analysis, showing that the total non-esterified fatty acids were increased by alcohol exposure and decreased by LGGs supplementation. Histological examination confirmed our metabolomics finding that hepatic fat accumulation by alcohol was markedly increased, and LGGs supplementation prevented it (Figure 23).

Among six amino acids, the abundances of five amino acids including L-isolucine, L-proline, L-threonine, L-phenylalanine and L-valine were decreased when mice were exposed to alcohol alone (column FC-2 in Table 11), while they all increased when the alcohol fed mice were exposed to LGGs (column FC-1 in Table 11).

Table 11. Metabolites with significant changes of abundance level among the four liver sample groups.

\begin{tabular}{|c|c|c|c|c|c|c|c|c|c|}
\hline Name & CAS & ${ }^{1} t_{R}(s)$ & ${ }^{2} t_{R}(s)$ & FC-1 ${ }^{\mathrm{a}}$ & FC-2 ${ }^{b}$ & FC- $3^{c}$ & $p-1^{d}$ & $p-2^{e}$ & $p-3^{f}$ \\
\hline \multicolumn{10}{|l|}{ Fatty acids } \\
\hline Acetic acid ${ }^{g}$ & $67226-76-0$ & 1443 & 1.01 & -1.05 & 2.05 & 1.22 & $1.3 \mathrm{E}-03$ & $9.0 \mathrm{E}-01$ & 2.9E-01 \\
\hline Dodecanoic acid & $143-07-7$ & 1961 & 1.04 & -1.41 & 1.59 & -1.09 & 8.9E-03 & 7.6E-02 & 4.7E-01 \\
\hline Hexanoic acid & $142-62-1$ & 1113 & 1.00 & -1.23 & 1.56 & -1.09 & $2.1 \mathrm{E}-03$ & $2.4 \mathrm{E}-01$ & 8.1E-01 \\
\hline Tetradecanoic acid & $4544-63-8$ & 2196 & 1.06 & -1.62 & 1.42 & 1.20 & $9.0 \mathrm{E}-01$ & 4.4E-01 & $4.4 \mathrm{E}-02$ \\
\hline $\begin{array}{l}\text { cis-9-Hexadecenoic } \\
\text { acid }^{\text {g }}\end{array}$ & $373-49-9$ & 2385 & 1.11 & -1.40 & 2.25 & 1.26 & $8.2 \mathrm{E}-03$ & $9.9 \mathrm{E}-01$ & $1.0 \mathrm{E}-01$ \\
\hline $\begin{array}{l}\text { trans-9-Octadecenoi } \\
\text { c } \text { acid }^{\mathrm{g}}\end{array}$ & $112-79-8$ & 2594 & 1.17 & -2.44 & -1.28 & -2.28 & $3.6 \mathrm{E}-01$ & $3.1 \mathrm{E}-02$ & 8.5E-01 \\
\hline Mead acid ${ }^{\mathrm{g}}$ & $20590-32-3$ & 2793 & 1.51 & 1.01 & 1.82 & 1.59 & $3.5 \mathrm{E}-02$ & 2.7E-01 & $1.7 \mathrm{E}-01$ \\
\hline Arachidonic acid ${ }^{g}$ & $506-32-1$ & 2762 & 1.51 & 1.35 & -1.55 & 1.10 & $1.7 \mathrm{E}-02$ & 7.9E-02 & 4.0E-01 \\
\hline \multicolumn{10}{|l|}{ Amino acids } \\
\hline L -Isoleucine & $73-32-5$ & 1748 & 0.99 & 1.43 & -1.09 & 1.20 & $1.0 \mathrm{E}+00$ & $3.9 \mathrm{E}-03$ & $2.8 \mathrm{E}-01$ \\
\hline L-Proline & $147-85-3$ & 1803 & 1.08 & 1.33 & -1.46 & 1.13 & $1.7 \mathrm{E}-03$ & $2.2 \mathrm{E}-02$ & $2.5 \mathrm{E}-01$ \\
\hline L -Threonine & $72-19-5$ & 1769 & 1.05 & 1.20 & -1.08 & -1.18 & 7.1E-05 & $1.4 \mathrm{E}-01$ & $1.3 \mathrm{E}-02$ \\
\hline L-Phenylalanine & $63-91-2$ & 2189 & 1.16 & 1.28 & -1.10 & 1.31 & $3.2 \mathrm{E}-01$ & 2.8E-02 & 9.9E-01 \\
\hline
\end{tabular}




\begin{tabular}{|c|c|c|c|c|c|c|c|c|c|}
\hline L-Tyrosine & $60-18-4$ & 2768 & 1.38 & 1.22 & 1.02 & 1.20 & $6.6 \mathrm{E}-01$ & $1.6 \mathrm{E}-02$ & $8.8 \mathrm{E}-01$ \\
\hline L-Valine & $72-18-4$ & 1652 & 0.99 & 1.34 & -1.06 & 1.16 & 9.7E-01 & $3.0 \mathrm{E}-02$ & 4.8E-01 \\
\hline \multicolumn{10}{|l|}{ Other acids } \\
\hline $\begin{array}{l}\text { 2-aminobutanoic } \\
\text { acid }\end{array}$ & $2835-81-6$ & 1585 & 0.99 & -2.16 & 3.57 & -1.14 & $2.5 \mathrm{E}-04$ & $1.3 \mathrm{E}-01$ & 8.0E-02 \\
\hline Glycolic acid ${ }^{\mathrm{g}}$ & $79-14-1$ & 1443 & 1.01 & -1.05 & 2.05 & 1.22 & $1.4 \mathrm{E}-03$ & $9.0 \mathrm{E}-01$ & $2.9 \mathrm{E}-01$ \\
\hline $\begin{array}{l}\alpha \text {-thylphenylacetic } \\
\text { acid }^{\mathrm{g}}\end{array}$ & $90-27-7$ & 2499 & 1.29 & 1.30 & -2.39 & 1.04 & $9.6 \mathrm{E}-04$ & 3.7E-01 & 7.2E-01 \\
\hline \multicolumn{10}{|l|}{ Others } \\
\hline 3Pyridinol ${ }^{\mathrm{g}}$ & $109-00-2$ & 1245 & 1.20 & -1.18 & 1.43 & 1.46 & $3.3 \mathrm{E}-01$ & $2.8 \mathrm{E}-01$ & $1.2 \mathrm{E}-02$ \\
\hline Hypoxanthine ${ }^{\mathrm{g}}$ & $68-94-0$ & 2345 & 1.43 & -1.09 & 1.55 & 1.26 & $3.2 \mathrm{E}-03$ & $5.1 \mathrm{E}-01$ & $9.4 \mathrm{E}-02$ \\
\hline $\begin{array}{l}\text { 2-(2-(2-ethoxyethox } \\
\text { y)ethoxy)acetic acid }\end{array}$ & $16024-58-1$ & 2456 & 1.05 & 4.20 & -4.04 & 1.14 & $4.3 \mathrm{E}-03$ & $3.9 \mathrm{E}-03$ & $1.5 \mathrm{E}-02$ \\
\hline
\end{tabular}

${ }^{a}$ fold change for AF-1 to AF-0. ${ }^{b}$ fold change for AF-0 to PF-0. ${ }^{c}$ fold change for PF-1 to PF-0. ${ }^{d}$-value of factor alcohol. ${ }^{e}$-value of factor LGGs. ${ }^{f}$-value of the interaction of alcohol and LGGs. ${ }^{\mathrm{g}}$ Tentative identification without verification using authentic standards. 


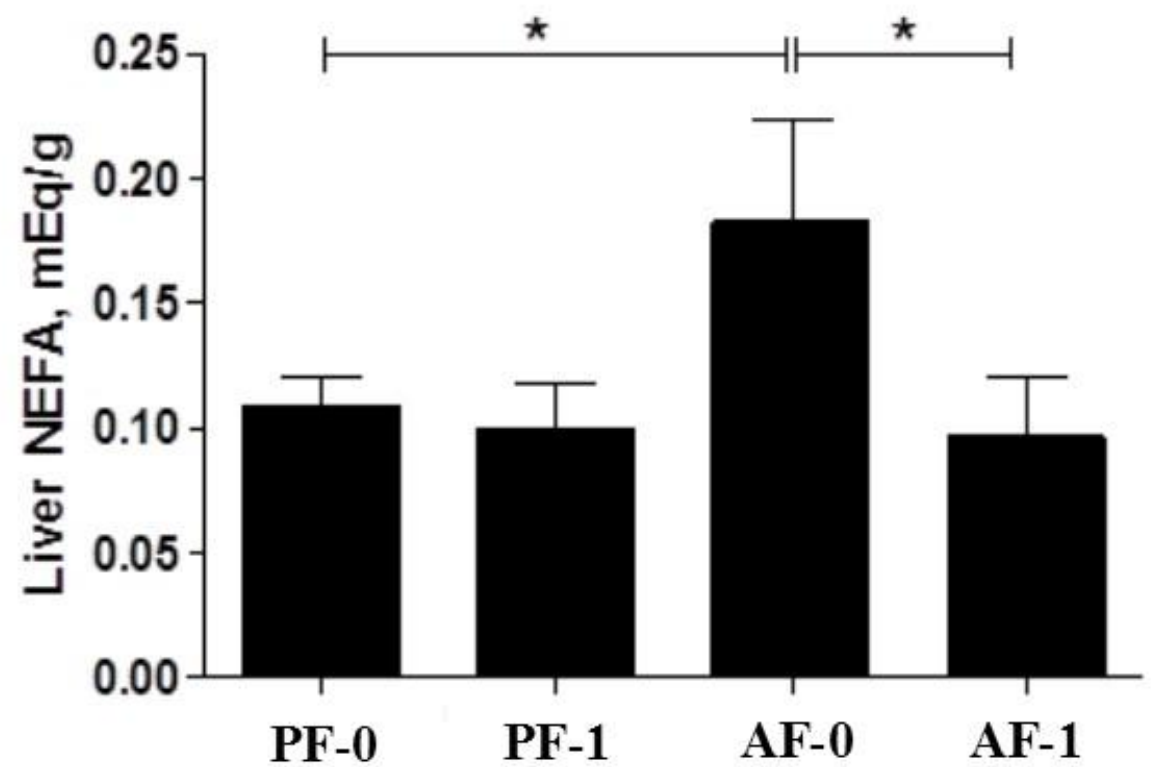

Figure 22. Effect of LGGs on hepatic free fatty acid levels. Stars * refer to the amount of total free fatty acids between two sample groups is significantly different with $\mathrm{p}<0.05$.

Table 12 lists metabolites with significant abundance changes between sample groups detected from fecal samples with a threshold of $p \leq 0.05$. A total of nine free fatty acids, nine amino acids, two alcohols, six other acids and nine other small compounds have significant changes in abundance level due to alcohol, LGGs or their synergetic interaction. Among these nine fatty acids, two are short chain fatty acids (SCFA, isovaleric acid and pentanoic acid), one MCFA (hexanoic acid), four LCFA (heptadecanoic acid, hexadecanoic acid, nonadecanoic acid, tetradecanoic acid), one polyunsaturated omega- 6 fatty acid (9,12-octadecadienoic acid) and one omega-7 monounsaturated fatty acid (cis-9-hexadecenoic acid). Compared with abundance levels in sample group AF-0, the abundance levels of these SCFAs, MCFAs and LCFAs in sample group AF-1 were significantly increased while the omega-7 monounsaturated fatty 
acid and polyunsaturated omega-6 fatty acid was decreased (column FC-1 in Table 12). Even though the fold-changes of most of the fatty acids are moderate, the abundance level of hexadecanoic acid was increased 20.6 fold in group AF-1. Among the 9 amino acids, the abundance levels of seven were decreased in group AF-0 compared to the group PF-0 (column FC-2 in Table 12), Among these seven amino acids, six amino acids were increased in the alcohol and LGGs treated group AF-1 compared to in the group AF-0 (column FC-1 in Table 12). It is interesting that the abundance level of L-Proline was decreased in group $\mathrm{AF}-1$ compared to the group $\mathrm{AF}-0$, decreased in group $\mathrm{AF}-0$ compared to group PF-0, and decreased in group PF-1 compared to that in group PF-0, respectively.

Table 12. Metabolites with significant change of abundance level among four fecal sample groups.

\begin{tabular}{|c|c|c|c|c|c|c|c|c|c|}
\hline Name & CAS & ${ }^{1} \mathbf{t}_{\mathrm{R}}(\mathbf{s})$ & ${ }^{2} t_{R}(s)$ & FC-1 ${ }^{\mathrm{a}}$ & FC-2 $^{b}$ & FC-3 $3^{\mathrm{c}}$ & $p-1^{d}$ & $p-2^{e}$ & $p-3^{f}$ \\
\hline \multicolumn{10}{|l|}{ Fatty acids } \\
\hline Isovaleric acid ${ }^{g}$ & 503-74-2 & 882 & 0.98 & 1.43 & -2.22 & 1.02 & $1.7 \mathrm{E}-02$ & $4.1 \mathrm{E}-01$ & $5.5 \mathrm{E}-01$ \\
\hline Pentanoic acid & $109-52-4$ & 957 & 1.00 & 1.67 & 1.26 & 1.96 & $5.7 \mathrm{E}-01$ & $1.7 \mathrm{E}-02$ & $5.9 \mathrm{E}-01$ \\
\hline Hexanoic acid & $142-62-1$ & 1114 & 1.02 & 1.58 & -1.25 & -1.54 & $5.5 \mathrm{E}-02$ & $9.2 \mathrm{E}-01$ & $3.2 \mathrm{E}-03$ \\
\hline Heptadecanoic acid & $506-12-7$ & 2511 & 1.12 & 1.25 & -2.41 & 1.44 & $1.5 \mathrm{E}-02$ & $1.5 \mathrm{E}-01$ & $5.5 \mathrm{E}-01$ \\
\hline Hexadecanoic acid & $57-10-3$ & 2396 & 1.10 & 20.6 & 2.04 & 8.74 & $1.3 \mathrm{E}-02$ & $1.5 \mathrm{E}-02$ & $6.5 \mathrm{E}-02$ \\
\hline Nonadecanoic acid & $646-30-0$ & 2728 & 1.31 & 1.06 & 1.69 & -1.06 & $1.2 \mathrm{E}-03$ & $9.6 \mathrm{E}-01$ & $6.2 \mathrm{E}-01$ \\
\hline Tetradecanoic acid & $544-63-8$ & 2197 & 1.09 & 1.81 & -1.41 & -1.01 & $4.5 \mathrm{E}-01$ & $1.6 \mathrm{E}-04$ & $1.4 \mathrm{E}-04$ \\
\hline $\begin{array}{l}\text { cis-9-Hexadecenoic } \\
\text { acid }^{\mathrm{g}}\end{array}$ & $373-49-9$ & 2385 & 1.14 & -2.84 & 1.88 & 1.67 & 4.7E-01 & $5.1 \mathrm{E}-01$ & $4.8 \mathrm{E}-02$ \\
\hline $\begin{array}{l}\text { 9,12-Octadecadienoi } \\
\text { c acid }^{\mathrm{g}}\end{array}$ & $60-33-3$ & 2687 & 1.37 & -1.82 & 1.02 & -1.88 & $8.8 \mathrm{E}-01$ & 4.1E-03 & $6.5 \mathrm{E}-01$ \\
\hline \multicolumn{10}{|l|}{ Amino acids } \\
\hline L-Methionine & $63-68-3$ & 2047 & 1.15 & 1.61 & -2.82 & 1.13 & $1.4 \mathrm{E}-04$ & $4.0 \mathrm{E}-02$ & $3.1 \mathrm{E}-01$ \\
\hline
\end{tabular}




\begin{tabular}{lccccccccc}
\hline L -Phenylalanine $^{-63-91-2}$ & 2189 & 1.20 & 1.99 & -2.57 & -1.13 & $3.6 \mathrm{E}-03$ & $1.4 \mathrm{E}-01$ & $4.4 \mathrm{E}-02$ \\
L -Ornithine $^{\mathrm{g}}$ & $70-26-8$ & 2389 & 1.05 & 8.34 & -1.68 & 1.13 & $3.4 \mathrm{E}-01$ & $1.1 \mathrm{E}-02$ & $4.5 \mathrm{E}-02$ \\
L -Alanine & $56-41-7$ & 789 & 0.97 & 1.66 & -1.18 & 1.47 & $3.9 \mathrm{E}-01$ & $2.0 \mathrm{E}-02$ & $9.1 \mathrm{E}-01$ \\
L -Threonine & $72-19-5$ & 2101 & 1.01 & 1.04 & -7.26 & 1.15 & $6.6 \mathrm{E}-06$ & $7.9 \mathrm{E}-01$ & $6.2 \mathrm{E}-01$ \\
L-Aspartic acid & $56-84-8$ & 2255 & 1.08 & 1.58 & -1.51 & -1.68 & $6.1 \mathrm{E}-01$ & $9.4 \mathrm{E}-01$ & $4.8 \mathrm{E}-02$ \\
L-Valine & $72-18-4$ & 1667 & 0.99 & 1.17 & 27.6 & 2.33 & $2.6 \mathrm{E}-06$ & $4.4 \mathrm{E}-03$ & $2.9 \mathrm{E}-02$ \\
L -Proline & $147-85-3$ & 1803 & 1.11 & -1.10 & -1.33 & -1.11 & $3.7 \mathrm{E}-03$ & $2.7 \mathrm{E}-01$ & $9.6 \mathrm{E}-01$ \\
L -Glutamic acid & $56-86-0$ & 2381 & 1.10 & -1.10 & 1.76 & -1.22 & $3.2 \mathrm{E}-02$ & $7.1 \mathrm{E}-01$ & $6.6 \mathrm{E}-01$
\end{tabular}

\section{Other acids}

\begin{tabular}{|c|c|c|c|c|c|c|c|c|c|}
\hline $\begin{array}{l}\text { 4-hydroxybenzoic } \\
\text { acid }^{\mathrm{g}}\end{array}$ & $99-96-7$ & 2231 & 1.19 & 3.12 & -2.83 & -1.10 & $1.5 \mathrm{E}-01$ & 4.9E-02 & $6.0 \mathrm{E}-02$ \\
\hline trans-Crotonic acid ${ }^{\mathrm{g}}$ & $107-93-7$ & 888 & 1.05 & 1.59 & -1.31 & -1.01 & $6.1 \mathrm{E}-01$ & 8.9E-03 & 5.9E-03 \\
\hline p-Coumaric acid ${ }^{g}$ & $501-98-4$ & 2579 & 1.32 & 1.46 & 2.34 & -1.43 & $7.0 \mathrm{E}-03$ & 7.5E-01 & $2.6 \mathrm{E}-01$ \\
\hline 2-ketobutyric acid ${ }^{\mathrm{g}}$ & $600-18-0$ & 1735 & 1.07 & -1.11 & 3.37 & 1.72 & $2.6 \mathrm{E}-03$ & $3.3 \mathrm{E}-01$ & 2.4E-01 \\
\hline Nicotinic acid ${ }^{g}$ & $59-67-6$ & 1509 & 1.28 & -1.07 & 6.29 & 1.12 & $1.6 \mathrm{E}-05$ & $6.7 \mathrm{E}-01$ & $8.6 \mathrm{E}-01$ \\
\hline Nonanedioic acid ${ }^{\mathrm{g}}$ & $123-99-9$ & 2391 & 1.17 & 1.89 & 1.42 & 1.14 & $3.0 \mathrm{E}-03$ & $5.2 \mathrm{E}-02$ & $1.6 \mathrm{E}-01$ \\
\hline \multicolumn{10}{|l|}{ Alcohols } \\
\hline Propylene glycol $^{\mathrm{g}}$ & $57-55-6$ & 1311 & 0.93 & 1.94 & -3.15 & -1.10 & $7.9 \mathrm{E}-05$ & 4.2E-02 & $1.1 \mathrm{E}-02$ \\
\hline $\begin{array}{l}\text { 2-Hydroxybenzyl } \\
\text { alcohol }^{\mathrm{g}}\end{array}$ & $90-01-7$ & 1961 & 1.13 & 1.46 & 2.36 & -1.36 & $6.6 \mathrm{E}-06$ & $6.5 \mathrm{E}-01$ & $1.3 \mathrm{E}-02$ \\
\hline \multicolumn{10}{|l|}{ Others } \\
\hline Phenol & $108-95-2$ & 1100 & 1.13 & 1.51 & -2.88 & -1.04 & $2.4 \mathrm{E}-05$ & $1.0 \mathrm{E}-01$ & 3.9E-02 \\
\hline DL-Glyceraldehyde ${ }^{\mathrm{g}}$ & $56-82-6$ & 2622 & 1.18 & -1.60 & -2.31 & 1.25 & $2.2 \mathrm{E}-02$ & 7.7E-01 & $8.1 \mathrm{E}-01$ \\
\hline Hydroxylamine $e^{g}$ & $\begin{array}{l}7803-49- \\
8\end{array}$ & 1076 & 0.94 & 1.58 & -2.12 & -1.23 & $1.4 \mathrm{E}-03$ & $2.2 \mathrm{E}-01$ & 7.1E-03 \\
\hline $\begin{array}{l}\text { Ethanolamine, } \\
\text { N-acetyl- }{ }^{\mathrm{a}}\end{array}$ & $142-26-7$ & 965 & 1.04 & 1.58 & -1.68 & -1.02 & $2.9 \mathrm{E}-02$ & 7.1E-02 & $5.0 \mathrm{E}-02$ \\
\hline $\begin{array}{l}\text { 2-(2-(2-ethoxyethoxy } \\
\text { )ethoxy)acetic acid }{ }^{\mathrm{g}}\end{array}$ & $\begin{array}{l}16024-58 \\
-1\end{array}$ & 2455 & 1.07 & -2.49 & -1.13 & -1.07 & $2.9 \mathrm{E}-02$ & 4.3E-01 & $6.1 \mathrm{E}-01$ \\
\hline $\begin{array}{l}\text { 1,2-Pyrrolidinedicarb } \\
\text { oxylic acid, }(2 \mathrm{R}) \mathrm{-}^{\mathrm{g}}\end{array}$ & $\begin{array}{l}1067236- \\
75-2\end{array}$ & 2139 & 1.29 & 1.70 & -1.08 & 1.22 & 5.3E-01 & $2.1 \mathrm{E}-02$ & 4.0E-01 \\
\hline Urea & $57-13-6$ & 1657 & 1.17 & 1.86 & 2.00 & 1.77 & 8.7E-03 & $4.9 \mathrm{E}-02$ & $9.0 \mathrm{E}-01$ \\
\hline 3-Pyridinol ${ }^{\mathrm{g}}$ & $109-00-2$ & 1061 & 1.14 & 1.94 & 1.83 & 1.32 & $1.1 \mathrm{E}-04$ & $2.5 \mathrm{E}-03$ & $8.6 \mathrm{E}-02$ \\
\hline $\mathrm{D}, \mathrm{L}-$ Homoserine ${ }^{\mathrm{g}}$ & $\begin{array}{l}1927-25- \\
9\end{array}$ & 2187 & 1.02 & -1.67 & 2.31 & -1.94 & $2.2 \mathrm{E}-02$ & $1.5 \mathrm{E}-01$ & 4.3E-01 \\
\hline
\end{tabular}


${ }^{\text {a }}$ fold change for AF-1 to AF-0. ${ }^{b}$ fold change for AF-0 to PF-0. ${ }^{c}$ fold change for PF-1 to PF-0. ${ }^{d}$-value of factor alcohol. ${ }^{e} p$-value of factor LGGs. ${ }^{f}$-value of the interaction of alcohol and LGGs. ${ }^{\mathrm{g}}$ Tentative identification without verification using authentic standards.
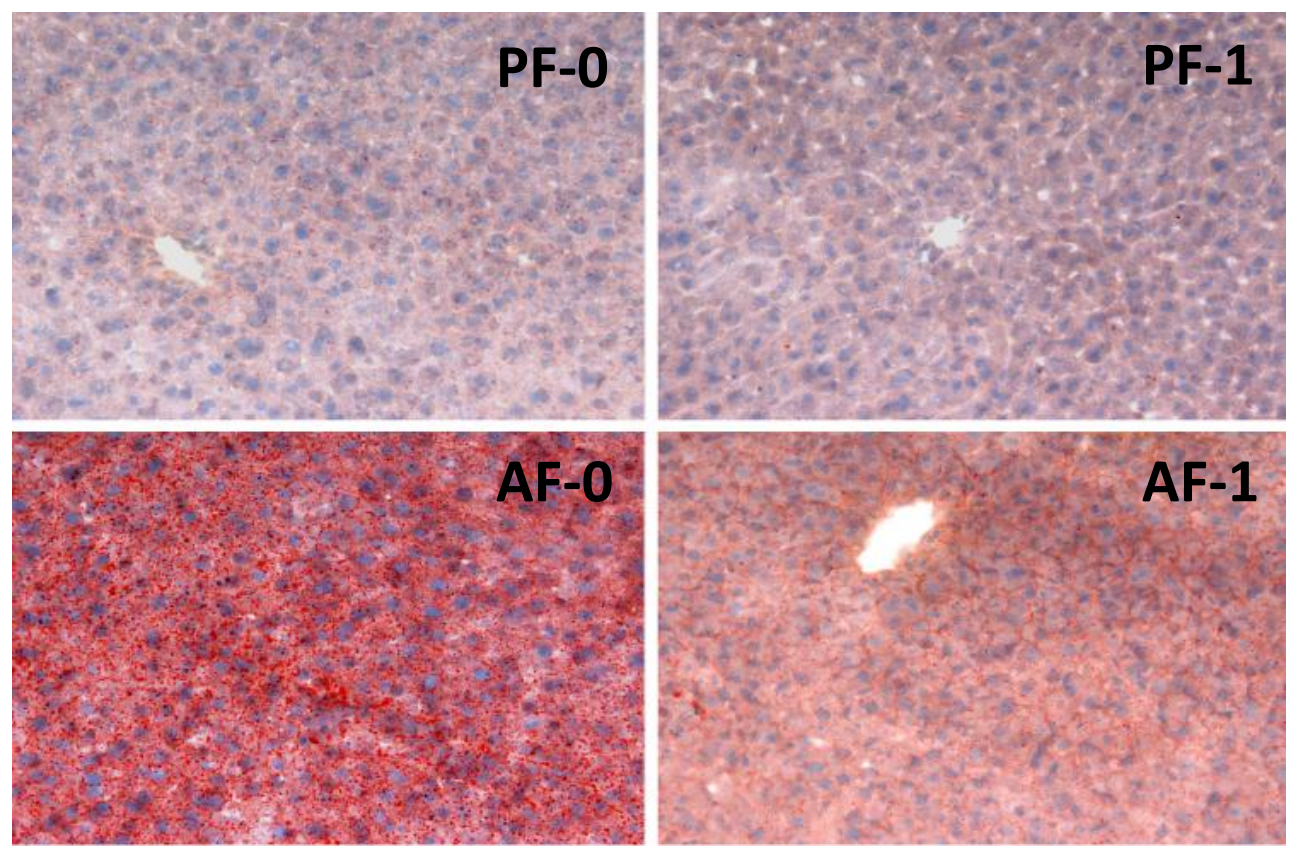

Figure 23. Effect of LGGs on liver fat accumulation. Hepatic cryosections were Oil red O stained and images were acquired by light microscopy.

\subsection{Discussion}

Four sample groups were formed in this study to investigate the effect of alcohol, LGGs and their interaction on the mouse liver and fecal metabolome, respectively. Figure 24 depicts clustering results of the metabolite profiles of the four liver sample groups (Figure 24A) and four fecal sample groups (Figure 24B), using partial least squares discriminant analysis (PLSDA). The clear separation between sample groups indicates that significant metabolic profiling difference presents between the sample groups. 

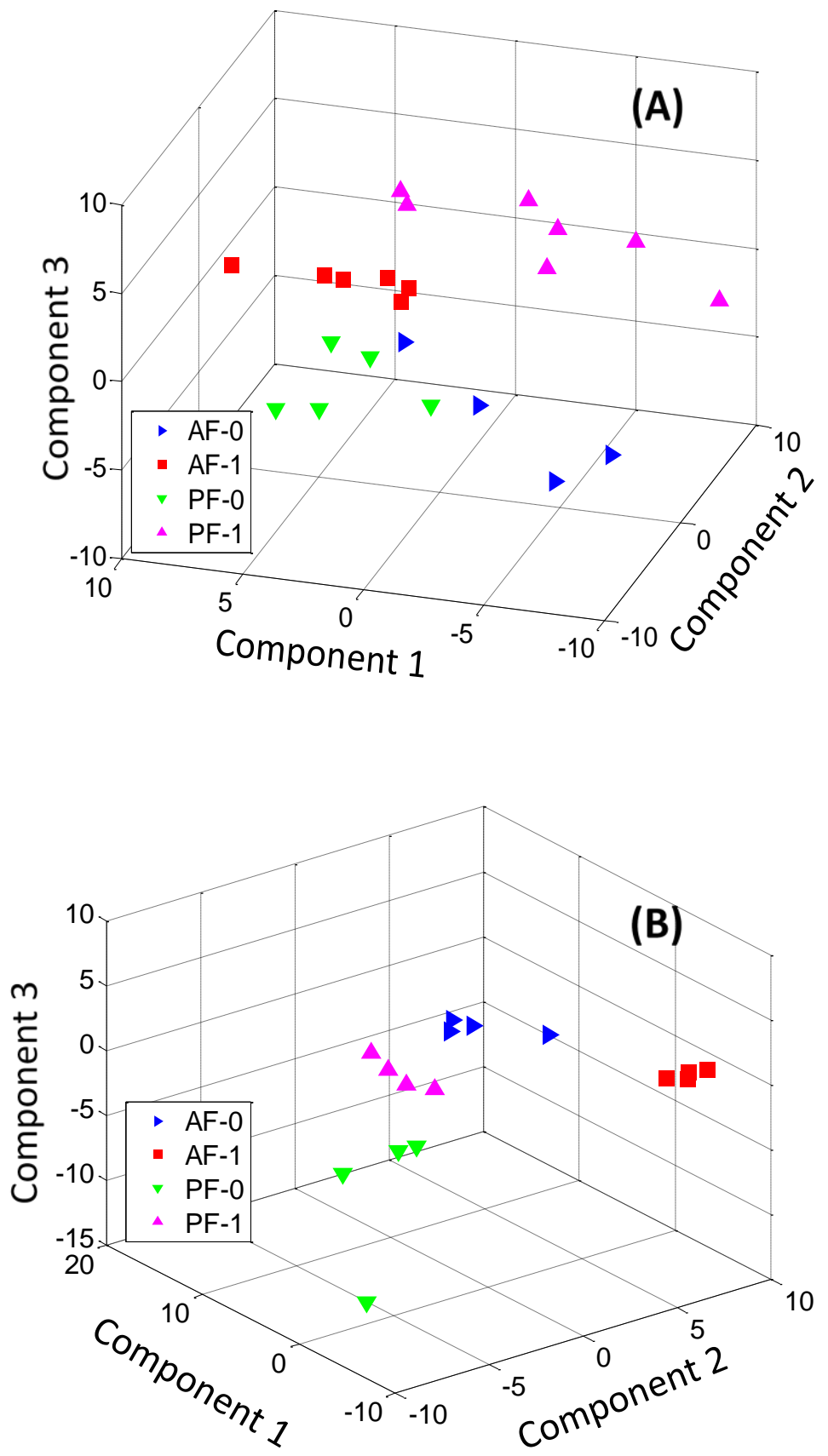

Figure 24. Clustering of metabolite profiles using partial least squares discriminant analysis (PLSDA). (A) is the clustering result of metabolite profiles of all liver samples and (B) is the clustering result of all fecal samples. 


\section{$\underline{\text { Analysis of liver samples }}$}

Excess alcohol consumption is one of the major factors in the development of fatty liver, which is characterized by hepatic accumulation of triglyceride, cholesterol and other types of lipids. A total of eight fatty acids were detected with significant alteration of abundance levels among the four sample groups. The four saturated fatty acids and cis-9-hexadecenoic acid have the same trend in alteration of abundance levels, while the polyunsaturated fatty acid arachidonic acid has opposite direction of abundance changes. The abundance levels of all four saturated fatty acids and cis-9-hexadecenoic acid are all increased in liver in the mice fed with alcohol. While SCFAs are metabolized in the mitochondria for energy production, MCFAs and LCFAs are mainly oxidized in the peroxisome[180].The higher levels of MCFAs and the LCFAs in the livers of mice fed alcohol strongly suggest that alcohol feeding interferes with lipid perexisomal oxidation, which is critical for hepatic fat elimination. However, hepatic levels of these fatty acids were significantly decreased in liver when LGGs was supplemented to alcohol-fed mice. This agrees with our total non-esterified fatty acids analysis and the results of histological examination of the liver sections (Figures 22 and 23). Taken together, the beneficial effects of LGGs on liver fat accumulation are mediated, at least in part, by increasing fatty acid peroxisomal oxidation.

Another finding in current study is the decrease of five amino acids, L-isoleucine, L-proline, L-threonine, L-phenylalanine, L-valine, by alcohol feeding, and the normalization of their abundance levels by LGGs supplementation. L-threonine is an essential amino acid which is not synthesized in mice. L-Threonine deficiency causes fat build up in the liver, while supplementation with L-threonine lowers hepatic fat 
concentration. [181]. L-Proline is a nonessential amino acid which can be synthesized from glutamate. Importantly, L-isoleucine is a branched-chain amino acid (BCAA), which is an essential amino acid and plays an important role in many aspects of hepatic physiology[182]. Clinical studies showed that BCAA enriched nutritional supplementation is beneficial for the treatment of ALD[183].Our results agree with these studies and demonstrate that LGGs supplementation increases L-isoleucine concentration to reduce the fat buildup induced by alcohol, likely through improved intestinal absorption. Altogether, our studies demonstrate that LGGs helps remedy the liver damage of amino acid depletion caused by alcohol consumption.

\section{Analysis of fecal samples}

We have previously demonstrated that saturated fat supplementation protects liver from alcohol-induced injury through stabilizing intestinal tight junctions leading to reduced circulating lipopolysaccharide (LPS)[150]. Although the underlying mechanisms are still unclear, recent studies showed that saturated fatty acids were metabolized by commensal Lactobacilli to promote their expansion[184]. Fatty acids can be synthesized by bacteria in the host in addition to being derived from the fat in the diet. In the current studies, we fed mice with the same amount of fat in the isocaloric diet in all animal groups. Therefore, the changes of fatty acids we observed are likely from bacterial production. The concentrations of five of nine detected fatty acids (i.e., isovaleric acid, hexanoic acid, heptadecanoic acid, tetradecanoic acid and cis-9-hexadecenoic acid) were significantly reduced in the feces of mice fed alcohol. Of particular interest, hepatadecanoic acid (C17:0), which is a LCFA produced only by bacteria[185] was reduced 2.41 folds by 
alcohol. Importantly, LGGs supplementation to both alcohol-fed and pair-fed mice significantly increased luminal levels of hepatadecanoic acid.

Chronic alcohol exposure significantly changes the gut bacterial population[186]. Administration of probiotics to alcohol-exposed mice normalized gut microflora, restored intestinal barrier tight junctions, reduced endotoxin translocation and attenuated liver steatosis and inflammation[169]. The increased abundance levels of fatty acids (i.e., isovaleric acid, pentanoic acid, hexanoic acid, heptadecanoic acid, hexadecanoic acid, nonadecanoic acid and tetradecanoic acid), therefore, contributes to the beneficial effects of LGGs in the reduced fatty liver and liver injury in response to alcohol exposure.

Similar to our findings in the liver, fecal concentrations of six amino acids (L-methionine, L-phenylalanine, L-omithine, L-alanine, L-threonine and L-aspartic acid) were reduced by alcohol feeding and increased by LGGs supplementation. Proteins in the diet are degraded in the intestine to amino acids which serve as nutrients for intestinal cells and extraenteric tissues. Previous work demonstrated that gut microflora potentially play a major role in the proteolysis in the large intestine. Amino acid metabolism in the host cells depends on the types of bacteria and the amino acids. Currently, it is still unclear how a specific amino acid is metabolized in the gut in response to alcohol exposure. The fact that the decreased amino acid concentrations of six amino acids were increased by LGGs supplementation suggests that the effects of LGGs on amino acid anabolism could potentially contribute to the beneficial effects in ALD.

While metabolite regulation differences between sample groups observed in this study are consistent with our histological study, our current work still has some limitations. Metabolites in mouse liver and mouse feces can have very diverse chemical 
characteristics. In this work, metabolites were extracted by water and methanol. Most of the nonpolar metabolites were lost during this analytical step. The extracted metabolites were then analyzed on GC×GC-TOF MS. Even though GC×GC-TOF MS has better separation power compared to the conventional one dimensional GC-MS, it may not be enough to resolve all metabolites and may result in overlapping chromatographic peaks which would introduce significant challenges for metabolite identification and quantification. In this work, metabolite identification was first achieved by matching the experimental mass spectra to the mass spectra recorded in the NIST11 MS library. The incompleteness of the existing mass spectral library not only introduces a certain degree of false-positive identifications, but also leaves a number of chromatographic peaks without any compound identification. Besides the MS library, metabolite identification GC $\times$ GC-TOF MS can also be affected by the spectral similarity measure and the optimal weight factor used during mass spectral matching[139]. It is impossible to estimate the rate of false-positive identifications induced by the incompleteness of the NIST11 MS library, However, analysis of NIST MS library demonstrates that the accuracy mass spectral matching based metabolite identification can be improved from about $80 \%$ to $96 \%$ if the top five ranked metabolite candidates are considered as the identification results[139]. To increase the metabolite identification accuracy of using existing NIST11 MS library, the top five ranked metabolite candidates for each chromatographic peak were used as the results of mass spectral matching and further subjected to retention index matching in this study. The metabolite candidate with the best retention index matching was kept for further analysis. All of these technical limitations in the current study prevent us from seeing the entire picture of the metabolite profile in mouse liver 
and feces. Further studies, such as using different extraction methods and combining both GC $\times$ GC-TOF MS with two-dimensional liquid chromatography high resolution mass spectrometry (2D LC-MS) may provide more comprehensive results.

\subsection{Conclusions}

GC $\times$ GC-TOF MS was used to identify metabolites that have significant alteration in their abundance levels in mouse liver and fecal samples collected from mice fed with and without alcohol, with and without co-exposure to LGGs. Our results show that the abundance levels of saturated fatty acids increase in liver samples but the abundance levels of a fraction of these fatty acids decreased in fecal samples when the mice were fed with alcohol. However, the abundance levels of all saturated fatty acids were decreased in mouse livers but increased in mouse feces when the mice were co-exposed to LGGs. Also we found that alcohol consumption depletes some amino acids in mouse livers, and such liver damage can be remedied by supplementing the mice with LGGs. These results demonstrate that LGGs alleviates alcohol-induced fatty liver by the mechanisms involving increasing intestinal and decreasing hepatic fatty acids and increasing amino acid concentration. 


\section{CHAPTER 6 SUMMARY AND CONCLUSIONS}

In this dissertation we first developed a bioanalytical platform LTQ-FTICR MS coupled with DI-nESI and the associated bioinformatic platform MetSign for metabolite biomarker discovery. We further applied the developed platforms for metabolite biomarker discovery and mechanistic studies including the analysis of Triacylglycerols Using High-Resolution 
Mass Spectrometry in Combination with in vivo Metabolite Deuterium Labeling; Metabolomic Analysis of the Effects of Polychlorinated Biphenyls in Non-Alcoholic Liver Disease.

The obvious disadvantage of direct infusion MS is easily getting ion suppression since there is no separation before detection. However, LTQ-FTICR MS has the advantages of high sensitivity and accuracy, wide dynamic range, and the ability to identify metabolites from complex samples. Also, chip-based nano-spray ESI ionization can get rid of carry over. In chapter 2, we profile all measurable metabolites. Metabolites were extracted based on Bligh \& Dyer's method to cover both polar and non-polar metabolites. In chapter 3, we focus on triglycerides. Thus chloroform: methanol (2:1) was used for lipid extraction. After extraction, we optimized the instrument parameter settings and applied it to analyze biological sample.

MetSign software code was written by bioinformatics specialist from our group. The software has assignment and quantitative analysis function. During assignment analysis, one $\mathrm{m} / \mathrm{z}$ value gives more than one candidate. To narrow down the identification candidate, we used mass frontier software to get the theoretical MS/MS spectrum. At the same time, we did experiment to get the MS/MS spectrum of this particular $\mathrm{m} / \mathrm{z}$. By matching the similarities between the experimental spectrum and the theoretical MS/MS spectrum, we get the best matching ID. Then authentic standard was purchase and analyzed to get MS/MS spectrum to confirm our identification. By following this procedure, identification accuracy was greatly improved.

Also I conducted spike-in experiments to test the identification and quantitative accuracy. The standard provided the identification information required to test our identification 
accuracy. Spiking with different concentrations of standards provided quantitative results to test our accuracy of quantitative analysis. Overall, we improved the identification accuracy and quantitative accuracy of the direct infusion MS platform allowing it to be applied to biomarker discovery. However, biomarker clinical studies were not performed and the biomarker has not been validated in this work.

In Chapter 2, we applied the developed platform to metabolomic analysis of the effects of polychlorinated biphenyls in non-alcoholic liver disease. We found several significant changes in molecules for different group comparisons. Notably, erythronic acid had the largest abundance change with a 28.8 -fold increase in the HFD+PCB 153 group when compared to the HFD group. GSH had a 5.91-fold decrease in the HFD+PCB 153 group and its conjugate, S-(hydroxymethyl)glutathione had a 2.97-fold decrease. Based on these results, antioxidant depletion is likely to be an important consequence of this interaction. Our metabolomics study can give more detailed information and even demonstrate some mechanisms by comparing histological studies.

In Chapter 3, we applied the developed platform combing with in vivo Metabolite Deuterium Labeling to study deuterium labeled triglycerides. Deuterium labeling water was used to feed mice, which make sure triglycerides in liver will be deuterium labeled. After 5 weeks feeding, normal water will be used to feed mice. Liver tissue and white adipose tissue were both collected to study. Time course study were also conducted, the mice were sacrificed after 2 weeks feeding or after 4 weeks feeding. We found that deuterated triacylglycerols (TGs) were significantly increased in alcohol induced fatty liver at two and four weeks of alcohol feeding periods, Also found that deuterated TGs were significantly decreased in both eWAT and sWAT at the two and four weeks. The 
increase of deuterium incorporated TGs in alcohol-induced fatty liver and their decrease in both eWAT and sWAT indicate that alcohol exposure induces hepatic influx of fatty acids which are released from WATs. The time course results further indicate a mechanistic link between adipose fat loss and hepatic fat gain in alcoholic fatty liver. This mechanism cannot be found only by histological study. Deuterium labeling combining with our analytical platform were combined together to demonstrate the mechanism.

We also successfully developed a GC×GC-TOF MS bioanalytical platform and MetPP bioinformatics platform for metabolomic profiling, and applied them to metabolite biomarker discovery including the effects of Chronic Arsenic Exposure in a Mouse Model of Diet-induced Fatty Liver Disease; Hepatic and Fecal Metabolomic Analysis of the Effects of Lactobacillus Rhamnosus GG on Alcoholic Fatty Liver Disease in Mice. GC $\times$ GC was originally described in 1991 . However, the system is still not that widely used mainly due to two reasons. One is the system is much complicated, another reason is the bottleneck of processing high throughput of $\mathrm{GC} \times \mathrm{GC}$ MS data. $\mathrm{GC} \times \mathrm{GC}-\mathrm{TOF}$ MS comparing with GC-TOF MS, it increased peak capacity, peak resolution, peak sensitivity, dynamic range, signal to noise ration when the system is optimized. However, the system is more complicated comparing with GC-TOF MS. A lot of parameters including column configuration including column stationary phase, column length, column width, temperature gradient, temperature offset of two column, flow rate, hot and cold pulse time ratio, modulation period will affect the separation power of the system. It is very critical to optimize all the parameters to get good metabolites coverage before sample analysis. We optimized each parameter based on peak resolution, detection limit, whether wrap around exist or not. 
For data processing, currently commercially software like ChromaTOF can do peak picking and tentative metabolite identification based on similarity between EI spectrum with NIST database. However, there is relative high rate of false identification. Retention index matching was performed using iMatch software with $\mathrm{p}$-value threshold set as $\mathrm{p} \leq$ 0.001, which use the information of retention time based on chemical property. Parts of the identified metabolites were confirmed by the first dimension retention index matching and parts were also preserved due to the lack of retention index information in the NIST11 retention index database. However, amount of the mass spectral matched metabolites were removed due to the large first dimension retention index deviation from the database values. This will decrease ratio of false identification. After this step, commercially available authentic standards were also purchased and analyzed to get spectrum to confirm our identification. Some metabolites were tentatively assigned if there are no commercially available standards. Tentatively-identified metabolites were considered as false identifications and removed from the identification list, if they were recognized as drugs or from non-mammalian animals during metabolic network analysis by the Ingenuity Pathway Analysis (IPA) software (Ingenuity Systems, Inc., Redwood City, CA).

Spike-in experiments were also carried out to test the identification and quantitative accuracy. The standard can give the identification information to test our identification accuracy. Spiking with different concentration of standards can provide quantitative results to test our accuracy of quantitative analysis.

In Chapter 4, we applied the developed platform to metabolomic analysis of the effects of chronic arsenic exposure of diet induced fatty liver disease. We optimized our system before our sample analysis and we did spike-in experiment to test the accuracy of 
identification and quantitation before sample analysis. A high value of AUC indicates a high accuracy of recognizing the concentration difference of the spiked-in compounds between sample groups.

We found that the high fat diet significantly altered nine metabolites and the addition of arsenic resulted in further alterations in three of these metabolites. The HFD-induced increase in cysteine was amplified, whereas the increase in glutamate was blocked, by co-exposure to arsenic. Also, the HFD-induced decrease in lysine was reversed by arsenic. Glycine was decreased by the combination of arsenic and a high fat diet. Therefore, all three of the amino acids that constitute the antioxidant glutathione (glutamate, cysteine and glycine) were altered, with glutamate and glycine levels lower and cysteine levels higher. The results showed that there are distinct hepatic metabolomic profiles associated with eating a high fat diet, drinking arsenic contaminated water, and the combination of the two. Among the metabolites that were decreased when arsenic exposure was combined with a high fat diet were hepatoprotective shortchain and medium-chain fatty acid metabolites and the anti-inflammatory amino acid glycine. These results are consistent with the observed increase in inflammation and cell death in the livers of these mice and point to potentially novel mechanisms by which these metabolic pathways could be altered by arsenic in the context of diet-induced fatty liver disease.

In Chapter 5, the platforms were applied to hepatic and fecal metabolomic analysis of the effects of LGG on alcoholic fatty liver disease in mice. Analysis of NIST MS library demonstrates that the accuracy mass spectral matching based metabolite identification can be improved from about $80 \%$ to $96 \%$ if the top five ranked metabolite candidates are considered as the identification results. To increase the metabolite identification accuracy 
of using existing NIST11 MS library, the top five ranked metabolite candidates for each chromatographic peak were used as the results of mass spectral matching and further subjected to retention index matching.

Fecal sample and liver sample were both collected. After analysis, we found that saturated fatty acids increase in liver samples, but decreased in fecal samples when the mice were fed with alcohol. However, all saturated fatty acids were decreased in mouse livers but increased in mouse feces when the mice were co-exposed to LGGs. Alcohol consumption depletes some amino acids in mouse livers, and such liver damage can be remedied by supplementing the mice with LGGs. It demonstrates that LGGs alleviates alcohol-induced fatty liver by the mechanisms involving increasing intestinal and decreasing hepatic fatty acids and increasing amino acid concentration. 


\section{REFERENCES}

1. Zhao, Y. Y., Cheng, X. L., Vaziri, N. D., Liu, S., and Lin, R. C. (2014) UPLC-based metabonomic applications for discovering biomarkers of diseases in clinical chemistry, Clin Biochem.

2. Wishart, D. S., Knox, C., Guo, A. C., Eisner, R., Young, N., Gautam, B., Hau, D. D., Psychogios, N., Dong, E., Bouatra, S., Mandal, R., Sinelnikov, I., Xia, J. G., Jia, L., Cruz, J. A., Lim, E., Sobsey, C. A., Shrivastava, S., Huang, P., Liu, P., Fang, L., Peng, J., Fradette, R., Cheng, D., Tzur, D., Clements, M., Lewis, A., De Souza, A., Zuniga, A., Dawe, M., Xiong, Y. P., Clive, D., Greiner, R., Nazyrova, A., Shaykhutdinov, R., Li, L., Vogel, H. J., and Forsythe, I. (2009) HMDB: a knowledgebase for the human metabolome, Nucleic Acids Research 37, D603-D610.

3. Schmidt, R. H., Jokinen, J. D., Massey, V. L., Falkner, K. C., Shi, X., Yin, X. M., Zhang, X., Beier, J. I., and Arteel, G. E. (2013) Olanzapine Activates Hepatic Mammalian Target of Rapamycin: New Mechanistic Insight into Metabolic Dysregulation with Atypical Antipsychotic Drugs, Journal of Pharmacology and Experimental Therapeutics 347, 126-135.

4. Shi, X., Wahlang, B., Wei, X. L., Yin, X. M., Falkner, K. C., Prough, R. A., Kim, S. H., Mueller, E. G., McClain, C. J., Cave, M., and Zhang, X. (2012) Metabolomic Analysis of 
the Effects of Polychlorinated Biphenyls in Nonalcoholic Fatty Liver Disease, J Proteome Res 11, 3805-3815.

5. Shi, X., Wei, X., Koo, I., Schmidt, R. H., Yin, X., Vaughn, A., Kim, S. H., McClain, C. J., Arteel, G. E., Zhang, X., and Watson, W. H. (2014) Metabolomic analysis of the effects of chronic arsenic exposure in a mouse model of diet-induced fatty liver disease, J. Proteome Res. 13, 547-554.

6. Jin, S., Song, C., Li, S., Zhang, Y., Chen, C., Zhou, X., Xu, Y., Feng, Y., Zhang, Z., and Jiang, H. (2014) Preventive effects of turmeric on the high-fat diet-induced hyperlipidaemia in mice associated with a targeted metabolomic approach for the analysis of serum lysophosphatidylcholine using LC-MS/MS, J. Funct. Foods 11, $130-141$.

7. Calder, A. G., and Smith, A. (1988) Stable isotope ratio analysis of leucine and ketoisocaproic acid in blood plasma by gas chromatography/mass spectrometry. Use of tertiary butyldimethylsilyl derivatives, Rapid Commun. Mass Spectrom. 2, 14-16.

8. Baran, R., Bowen, B. P., Bouskill, N. J., Brodie, E. L., Yannone, S. M., and Northen, T. R. (2010) Metabolite Identification in Synechococcus sp. PCC 7002 Using Untargeted Stable Isotope Assisted Metabolite Profiling, Analytical Chemistry 82, 9034-9042.

9. Creek, D. J., Chokkathukalam, A., Jankevics, A., Burgess, K. E. V., Breitling, R., and Barrett, M. P. (2012) Stable Isotope-Assisted Metabolomics for Network-Wide Metabolic Pathway Elucidation, Analytical Chemistry 84, 8442-8447. 
10. Fan, T. W. M., Lane, A. N., Higashi, R. M., Farag, M. A., Gao, H., Bousamra, M., and Miller, D. M. (2009) Altered regulation of metabolic pathways in human lung cancer discerned by C-13 stable isotope-resolved metabolomics (SIRM), Molecular Cancer 8.

11. Bathen, T. F., Sitter, B., Sjobakk, T. E., Tessem, M. B., and Gribbestad, I. S. (2010) Magnetic resonance metabolomics of intact tissue: a biotechnological tool in cancer diagnostics and treatment evaluation, Cancer Res 70, 6692-6696.

12. Powers, R. (2009) NMR metabolomics and drug discovery, Magn Reson Chem 47 Suppl 1, S2-11.

13. Schripsema, J. (2010) Application of NMR in plant metabolomics: techniques, problems and prospects, Phytochem Anal 21, 14-21.

14. Viant, M. R., Rosenblum, E. S., and Tieerdema, R. S. (2003) NMR-based metabolomics: a powerful approach for characterizing the effects of environmental stressors on organism health, Environ Sci Technol 37, 4982-4989.

15. Ward, J. L., Baker, J. M., and Beale, M. H. (2007) Recent applications of NMR spectroscopy in plant metabolomics, FEBS J 274, 1126-1131.

16. Zhang, S., Nagana Gowda, G. A., Ye, T., and Raftery, D. (2010) Advances in NMR-based biofluid analysis and metabolite profiling, Analyst 135, 1490-1498.

17. Dieterle, F., Riefke, B., Schlotterbeck, G., Ross, A., Senn, H., and Amberg, A. (2011) NMR and MS methods for metabonomics, Methods Mol Biol 691, 385-415.

18. Bando, K., Kunimatsu, T., Sakai, J., Kimura, J., Funabashi, H., Seki, T., Bamba, T., and Fukusaki, E. (2010) GC-MS-based metabolomics reveals mechanism of action for hydrazine induced hepatotoxicity in rats, J Appl Toxicol. 
19. Cevallos-Cevallos, J. M., Garcia-Torres, R., Etxeberria, E., and Reyes-De-Corcuera, J. I. (2010) GC-MS Analysis of Headspace and Liquid Extracts for Metabolomic Differentiation of Citrus Huanglongbing and Zinc Deficiency in Leaves of 'Valencia' Sweet Orange from Commercial Groves, Phytochem Anal.

20. Lin, S., Liu, N., Yang, Z., Song, W., Wang, P., Chen, H., Lucio, M., Schmitt-Kopplin, P., Chen, G., and Cai, Z. (2010) GC/MS-based metabolomics reveals fatty acid biosynthesis and cholesterol metabolism in cell lines infected with influenza A virus, Talanta 83, 262-268.

21. Stein, S. (1999) An Integrated Method for Spectrum Extraction and Compound Identification from GC/MS Data, J. Am. Soc. Mass Spectrom. 10, 12.

22. Thysell, E., Surowiec, I., Hornberg, E., Crnalic, S., Widmark, A., Johansson, A. I., Stattin, P., Bergh, A., Moritz, T., Antti, H., and Wikstrom, P. (2010) Metabolomic characterization of human prostate cancer bone metastases reveals increased levels of cholesterol, PLoS One 5, e14175.

23. Tikunov, Y., Lommen, A., de Vos, C. H., Verhoeven, H. A., Bino, R. J., Hall, R. D., and Bovy, A. G. (2005) A novel approach for nontargeted data analysis for metabolomics. Large-scale profiling of tomato fruit volatiles, Plant Physiol 139, 1125-1137.

24. Lee do, Y., Bowen, B. P., and Northen, T. R. (2010) Mass spectrometry-based metabolomics, analysis of metabolite-protein interactions, and imaging, Biotechniques 49, $557-565$.

25. Garcia, A., and Barbas, C. (2011) Gas chromatography-mass spectrometry (GC-MS)-based metabolomics, Methods Mol Biol 708, 191-204. 
26. Kieken, F., Pinel, G., Antignac, J. P., Monteau, F., Christelle Paris, A., Popot, M. A., Bonnaire, Y., and Le Bizec, B. (2009) Development of a metabonomic approach based on LC-ESI-HRMS measurements for profiling of metabolic changes induced by recombinant equine growth hormone in horse urine, Anal Bioanal Chem 394, 2119-2128.

27. Moco, S., Bino, R. J., Vorst, O., Verhoeven, H. A., de Groot, J., van Beek, T. A., Vervoort, J., and de Vos, C. H. (2006) A liquid chromatography-mass spectrometry-based metabolome database for tomato, Plant Physiol 141, 1205-1218.

28. Mohamed, R., Varesio, E., Ivosev, G., Burton, L., Bonner, R., and Hopfgartner, G. (2009) Comprehensive analytical strategy for biomarker identification based on liquid chromatography coupled to mass spectrometry and new candidate confirmation tools, Anal Chem 81, 7677-7694.

29. Sana, T. R., Roark, J. C., Li, X., Waddell, K., and Fischer, S. M. (2008) Molecular formula and METLIN Personal Metabolite Database matching applied to the identification of compounds generated by LC/TOF-MS, J Biomol Tech 19, 258-266.

30. Zhang, A., Sun, H., Wang, P., Han, Y., and Wang, X. (2012) Modern analytical techniques in metabolomics analysis, Analyst 137, 293-300.

31. Price, P. (2000) Mass spectrometry desk reference, edited by O. David Sparkman, J. Am. Soc. Mass Spectrom. 11, 1144.

32. Mishur, R. J., and Rea, S. L. (2012) Applications of mass spectrometry to metabolomics and metabonomics: Detection of biomarkers of aging and of age-related diseases, Mass Spectrom Rev 31, 70-95. 
33.González-Domínguez, R., Castilla-Quintero, R., García-Barrera, T., and Gómez-Ariza, J. L. (2014) Development of a metabolomic approach based on urine samples and direct infusion mass spectrometry, Anal Biochem 465, 20-27.

34. Lokhov, P. G., Kharybin, O. N., and Archakov, A. I. (2012) Diagnosis of lung cancer based on direct-infusion electrospray mass spectrometry of blood plasma metabolites, Int J Mass Spectrom 309, 200-205.

35. Lerma-García, M. J., Herrero-Martínez, J. M., Ramis-Ramos, G., and Simó-Alfonso, E. F. (2008) Prediction of the genetic variety of Spanish extra virgin olive oils using fatty acid and phenolic compound profiles established by direct infusion mass spectrometry, Food Chem 108, 1142-1148.

36. Zhang, S., Van Pelt, C. K., and Henion, J. D. (2003) Automated chip-based nanoelectrospray-mass spectrometry for rapid identification of proteins separated by two-dimensional gel electrophoresis, Electrophoresis 24, 3620-3632.

37. Wilm, M., and Mann, M. (1996) Analytical properties of the nanoelectrospray ion source, Anal Chem 68, 1-8.

38. Weber, R. J. M., Southam, A. D., Sommer, U., and Viant, M. R. (2011) Characterization of Isotopic Abundance Measurements in High Resolution FT-ICR and Orbitrap Mass Spectra for Improved Confidence of Metabolite Identification, Anal Chem $83,3737-3743$.

39. Tan, A. M., Benetton, S., and Henion, J. D. (2003) Chip-based solid-phase extraction pretreatment for direct electrospray mass spectrometry analysis using an array of monolithic columns in a polymeric substrate, Anal Chem 75, 5504-5511. 
40. Pereira-Medrano, A. G., Sterling, A., SnijderS, A. P. L., Reardon, K. F., and Wright, P. C. (2007) A systematic evaluation of chip-based nanoelectrospray parameters for rapid identification of proteins from a complex mixture, J Am Soc Mass Spectr 18, 1714-1725.

41. Gabelica, V., Vreuls, C., Filee, P., Duval, V., Joris, B., and De Pauw, E. (2002) Advantages and drawbacks of nanospray for studying noncovalent protein-DNA complexes by mass spectrometry, Rapid Commun Mass Sp 16, 1723-1728.

42. Chen, J., Canales, L., and Neal, R. E. (2011) Multi-Segment Direct Inject nano-ESI-LTQ-FT-ICR-MS/MS For Protein Identification, Proteome Sci 9.

43. Theodoridis, G., Gika, H., Franceschi, P., Caputi, L., Arapitsas, P., Scholz, M., Masuero, D., Wehrens, R., Vrhovsek, U., and Mattivi, F. (2012) LC-MS based global metabolite profiling of grapes: solvent extraction protocol optimisation, Metabolomics 8, $175-185$.

44. Ressom, H. W., Xiao, J. F., Tuli, L., Varghese, R. S., Zhou, B., Tsai, T.-H., Nezami Ranjbar, M. R., Zhao, Y., Wang, J., Di Poto, C., Cheema, A. K., Tadesse, M. G., Goldman, R., and Shetty, K. (2012) Utilization of metabolomics to identify serum biomarkers for hepatocellular carcinoma in patients with liver cirrhosis, Anal Chim Acta $743,90-100$.

45. Chen, F., Xue, J., Zhou, L., Wu, S., and Chen, Z. (2011) Identification of serum biomarkers of hepatocarcinoma through liquid chromatography/mass spectrometry-based metabonomic method, Anal Bioanal Chem 401, 1899-1904. 
46. Liu, S.-Y., Zhang, R.-L., Kang, H., Fan, Z.-J., and Du, Z. (2013) Human liver tissue metabolic profiling research on hepatitis B virus-related hepatocellular carcinoma, World J. Gastroenterol. 19, 3423-3432.

47. Harn, H.-J., Chen, Y.-L., Lin, P.-C., Cheng, Y.-L., Lee, S.-C., Chiou, T.-W., and Yang, H.-H. (2010) Exploration of potential tumor markers for lung adenocarcinomas by two-dimensional gel electrophoresis coupled with nano-LC/MS/MS, J. Chin. Chem. Soc. (Taipei, Taiwan) 57, 180-188.

48. Yang, Q., Shi, X., Wang, Y., Wang, W., He, H., Lu, X., and Xu, G. (2010) Urinary metabonomic study of lung cancer by a fully automatic hyphenated hydrophilic interaction/RPLC-MS system, J. Sep. Sci. 33, 1495-1503.

49. Müller, D. C., Degen, C., Scherer, G., Jahreis, G., Niessner, R., and Scherer, M. (2014) Metabolomics using GC-TOF-MS followed by subsequent GC-FID and HILIC-MS/MS analysis revealed significantly altered fatty acid and phospholipid species profiles in plasma of smokers, Journal of Chromatography B 966, 117-126.

50. Lisa, M., Cifkova, E., and Holcapek, M. (2011) Lipidomic profiling of biological tissues using off-line two-dimensional high-performance liquid chromatography mass spectrometry, Journal of Chromatography A 1218, 5146-5156.

51. Fairchild, J. N., Horvath, K., Gooding, J. R., Campagna, S. R., and Guiochon, G. (2010) Two-dimensional liquid chromatography/mass spectrometry/mass spectrometry separation of water-soluble metabolites, Journal of Chromatography A 1217, 8161-8166.

52. Xiao, J. F., Zhou, B., and Ressom, H. W. (2012) Metabolite identification and quantitation in LC-MS/MS-based metabolomics, Trends Analyt Chem 32, 1-14. 
53. Zhang, T., and Watson, D. G. (2013) High performance liquid chromatographic approaches to mass spectrometry based metabolomics, Curr. Metabolomics 1, 58-83.

54. Zhang, G.-F., Li, Q., Li, L., and Kasumov, T. (2012) Metabolomics research with tandem mass spectrometry, pp 309-330, InTech.

55. Warth, B., Parich, A., Bueschl, C., Schoefbeck, D., Neumann, N. K. N., Kluger, B., Schuster, K., Krska, R., Adam, G., Lemmens, M., and Schuhmacher, R. (2014) GC-MS based targeted metabolic profiling identifies changes in the wheat metabolome following deoxynivalenol treatment, Metabolomics, Ahead of Print.

56. Kobayashi, T., Nishiumi, S., Kawana, S., Azuma, T., and Yoshida, M. (2013) Biomarker discovery for the pancreatic cancer by the serum metabolomics based on gas chromatography mass spectrometry, Shimadzu Hyoron 70, 117-121.

57. Schaffert, C. S., Duryee, M. J., Hunter, C. D., Hamilton, B. C., 3rd, DeVeney, A. L., Huerter, M. M., Klassen, L. W., and Thiele, G. M. (2009) Alcohol metabolites and lipopolysaccharide: roles in the development and/or progression of alcoholic liver disease, World J Gastroenterol 15, 1209-1218.

58. Blachier, M., Leleu, H., Peck-Radosavljevic, M., Valla, D.-C., and Roudot-Thoraval, F. (2013) The burden of liver disease in Europe: A review of available epidemiological data, J Hepatol 58, 593-608.

59. Ludwig, J., Viggiano, T. R., McGill, D. B., and Oh, B. J. (1980) Nonalcoholic steatohepatitis: Mayo Clinic experiences with a hitherto unnamed disease, Mayo Clin Proc 55, 434-438. 
60. Hashimoto, E., Taniai, M., and Tokushige, K. (2013) Characteristics and diagnosis of NAFLD/NASH, J Gastroenterol Hepatol 28 Suppl 4, 64-70.

61. Lin, X., Zhang, Y., Ye, G., Li, X., Yin, P., Ruan, Q., and Xu, G. (2011) Classification and differential metabolite discovery of liver diseases based on plasma metabolic profiling and support vector machines, J Sep Sci 34, 3029-3036.

62. Ji, P., Wei, Y., Sun, H., Xue, W., Hua, Y., Li, P., Zhang, W., Zhang, L., Zhao, H., and Li, J. (2014) Metabolomics research on the hepatoprotective effect of Angelica sinensis polysaccharides through gas chromatography-mass spectrometry, Journal of Chromatography B 973, 45-54.

63. Shi, X., Wei, X., Koo, I., Schmidt, R. H., Yin, X., Vaughn, A., Kim, S. H., McClain, C. J., Arteel, G. E., Zhang, X., and Watson, W. H. (2014) Metabolomic analysis of the effects of chronic arsenic exposure in a mouse model of diet-induced fatty liver disease, J. Proteome Res. 13, 547-554.

64. Morikawa, T., Ninomiya, K., Miyake, S., Miki, Y., Okamoto, M., Yoshikawa, M., and Muraoka, O. Flavonol glycosides with lipid accumulation inhibitory activity and simultaneous quantitative analysis of 15 polyphenols and caffeine in the flower buds of Camellia sinensis from different regions by LCMS, Food Chem 140, 353-360.

65. Trammell, S. A. J., and Brenner, C. (2013) TARGETED, LCMS-BASED METABOLOMICS FOR QUANTITATIVE MEASUREMENT OF NAD+ METABOLITES, Computational and Structural Biotechnology Journal 4, 1-9. 
66. MacIntyre, L., Zheng, L. A., Scullion, P., Keating, P., and Watson, D. G. (2011) Metabolomic profiling of biomarkers of liver $\mathrm{X}$ receptor-induced toxicity in mouse liver tissue, Metabolomics 7, 54-70.

67. Loftus, N., Barnes, A., Ashton, S., Michopoulos, F., Theodoridis, G., Wilson, I., Ji, C., and Kaplowitz, N. (2011) Metabonomic Investigation of Liver Profiles of Nonpolar Metabolites Obtained from Alcohol-Dosed Rats and Mice Using High Mass Accuracy MS(n) Analysis, J Proteome Res 10, 705-713.

68. Cave, M., Appana, S., Patel, M., Falkner, K. C., McClain, C. J., and Brock, G. (2010) Polychlorinated Biphenyls, Lead, and Mercury Are Associated with Liver Disease in American Adults: NHANES 2003-2004, Environ Health Persp 118, 1735-1742.

69. Lyche, J. L., Skaare, J. U., Larsen, H. J. S., and Ropstad, E. (2004) Levels of PCB 126 and PCB 153 in plasma and tissues in goats exposed during gestation and lactation, Chemosphere 55, 621-629.

70. Wahlang, B., Falkner, K., Conklin, D., McClain, C., and Cave, M. (2012) Polychlorinated biphenyl 153 worsens non-alcoholic fatty liver disease in C57BL/6 mice, The Toxicologist CD Supplement to Toxicological Sciences - An Official Journal of the Society of Toxicology. 126, 84 .

71. Zhong, W., Zhao, Y. T., Tang, Y. N., Wei, X. L., Shi, X., Sun, W. L., Sun, X. H., Yin, X. M., Sun, X. G., Kim, S., McClain, C. J., Zhang, X., and Zhou, Z. X. (2011) Chronic Alcohol Exposure Causes Adipose Fat Overflux to the Liver in Mice: A Mechanistic Link between Lipodystrophy and Steatosis, Hepatology 54, 978a-978a. 
72. Weber, R. J., Southam, A. D., Sommer, U., and Viant, M. R. (2011) Characterization of isotopic abundance measurements in high resolution FT-ICR and Orbitrap mass spectra for improved confidence of metabolite identification, Anal Chem 83, 3737-3743.

73. Higgs, R. E., Zahn, J. A., Gygi, J. D., and Hilton, M. D. (2001) Rapid method to estimate the presence of secondary metabolites in microbial extracts, Appl Environ Microbiol 67, 371-376.

74. Bradford, B. U., O'Connell, T. M., Han, J., Kosyk, O., Shymonyak, S., Ross, P. K., Winnike, J., Kono, H., and Rusyn, I. (2008) Metabolomic profiling of a modified alcohol liquid diet model for liver injury in the mouse uncovers new markers of disease, Toxicol Appl Pharm 232, 236-243.

75. Bligh, E. G., and Dyer, W. J. (1959) A rapid method of total lipid extraction and purification, Can J Biochem Physiol 37, 911-917.

76. Wei, X., Sun, W., Shi, X., Koo, I., Wang, B., Zhang, J., Yin, X., Tang, Y., Bogdanov, B., Kim, S., Zhou, Z., McClain, C., and Zhang, X. (2011) MetSign: a computational platform for high-resolution mass spectrometry-based metabolomics, Anal Chem 83, $7668-7675$.

77. Bolstad, B. M., Irizarry, R. A., Astrand, M., and Speed, T. P. (2003) A comparison of normalization methods for high density oligonucleotide array data based on variance and bias, Bioinformatics 19, 185-193.

78. Jahn, M., Baynes, J. W., and Spiteller, G. (1999) The reaction of hyaluronic acid and its monomers, glucuronic acid and $\mathrm{N}$-acetylglucosamine, with reactive oxygen species, Carbohydr Res 321, 228-234. 
79. Harding, J. J., Hassett, P. C., Rixon, K. C., Bron, A. J., and Harvey, D. J. (1999) Sugars including erythronic and threonic acids in human aqueous humour, Curr Eye Res $19,131-136$.

80. Kim, H. J., Kim, J. H., Noh, S., Hur, H. J., Sung, M. J., Hwang, J. T., Park, J. H., Yang, H. J., Kim, M. S., Kwon, D. Y., and Yoon, S. H. (2011) Metabolomic Analysis of Livers and Serum from High-Fat Diet Induced Obese Mice, J Proteome Res 10, 722-731.

81. Goncharov, A., Pavuk, M., Foushee, H. R., and Carpenter, D. O. (2011) Blood pressure in relation to concentrations of PCB congeners and chlorinated pesticides, Environ Health Perspect 119, 319-325.

82. Nehme, A., Lobenhofer, E. K., Stamer, W. D., and Edelman, J. L. (2009) Glucocorticoids with different chemical structures but similar glucocorticoid receptor potency regulate subsets of common and unique genes in human trabecular meshwork cells, Bmc Med Genomics 2.

83. Pompella, A., Visvikis, A., Paolicchi, A., De Tata, V., and Casini, A. F. (2003) The changing faces of glutathione, a cellular protagonist, Biochem Pharmacol 66, 1499-1503.

84. Kelly, A., and Stanley, C. A. (2001) Disorders of glutamate metabolism, Mental retardation and developmental disabilities research reviews 7, 287-295.

85. Hirano, T., Kaplowitz, N., Tsukamoto, H., Kamimura, S., and Fernandez-Checa, J. C. (1992) Hepatic mitochondrial glutathione depletion and progression of experimental alcoholic liver disease in rats, Hepatology 16, 1423-1427. 
86. Pastore, A., Ciampalini, P., Tozzi, G., Pecorelli, L., Passarelli, C., Bertini, E., and Piemonte, F. (2012) All glutathione forms are depleted in blood of obese and type 1 diabetic children, Pediatric Diabetes 13, 272-277.

87. Altomare, E., Vendemiale, G., and Albano, O. (1988) HEPATIC GLUTATHIONE CONTENT IN PATIENTS WITH ALCOHOLIC AND NONALCOHOLIC LIVER-DISEASES, Life Sci 43, 991-998.

88. Jeong, Y. C., Walker, N. J., Burgin, D. E., Kissling, G., Gupta, M., Kupper, L., Birnbaum, L. S., and Swenberg, J. A. (2008) Accumulation of M(1)dG DNA adducts after chronic exposure to PCBs, but not from acute exposure to polychlorinated aromatic hydrocarbons, Free Radical Bio Med 45, 585-591.

89. Lutz, R. J., Dedrick, R. L., Tuey, D., Sipes, I. G., Anderson, M. W., and Matthews, H. B. (1984) Comparison of the Pharmacokinetics of Several Polychlorinated-Biphenyls in Mouse, Rat, Dog, and Monkey by Means of a Physiological Pharmacokinetic Model, Drug Metab Dispos 12, 527-535.

90. Safe, S., Bandiera, S., Sawyer, T., Robertson, L., Safe, L., Parkinson, A., Thomas, P. E., Ryan, D. E., Reik, L. M., Levin, W., Denomme, M. A., and Fujita, T. (1985) PCBS STRUCTURE-FUNCTION-RELATIONSHIPS AND MECHANISM OF ACTION, Environ Health Persp 60, 47-56.

91. Twaroski, T. P., O'Brien, M. L., and Robertson, L. W. (2001) Effects of selected polychlorinated biphenyl (PCB) congeners on hepatic glutathione, glutathione-related enzymes, and selenium status: implications for oxidative stress, Biochemical Pharmacology 62, 273-281. 
92. Kalhan, S. C., Guo, L., Edmison, J., Dasarathy, S., McCullough, A. J., Hanson, R. W., and Milburn, M. (2011) Plasma metabolomic profile in nonalcoholic fatty liver disease, Metabolism 60, 404-413.

93. Barr, J., Vazquez-Chantada, M., Alonso, C., Perez-Cormenzana, M., Mayo, R., Galan, A., Caballeria, J., Martin-Duce, A., Tran, A., Wagner, C., Luka, Z., Lu, S. C., Castro, A., Le Marchand-Brustel, Y., Martinez-Chantar, M. L., Veyrie, N., Clement, K., Tordjman, J., Gual, P., and Mato, J. M. (2010) Liquid chromatography-mass spectrometry-based parallel metabolic profiling of human and mouse model serum reveals putative biomarkers associated with the progression of nonalcoholic fatty liver disease, $\mathbf{J}$ Proteome Res 9, 4501-4512.

94. Xiao, C., Hsieh, J., Adeli, K., and Lewis, G. F. (2011) Gut-liver interaction in triglyceride-rich lipoprotein metabolism, Am. J. Physiol. 301, E429-E446.

95. Sethi, J. K., and Vidal-Puig, A. J. (2007) Adipose tissue function and plasticity orchestrate nutritional adaptation, J. Lipid Res. 48, 1253-1262.

96. Cusi, K. (2010) The role of adipose tissue and lipotoxicity in the pathogenesis of type 2 diabetes, Curr. Diabetes Rep. 10, 306-315.

97. Bradbury MW, B. P. (2004) Lipid metabolism in hepatic steatosis, Clinics in liver disease 8, 639-671.

98. Sozio, M. S., Liangpunsakul, S., and Crabb, D. (2010) The role of lipid metabolism in the pathogenesis of alcoholic and nonalcoholic hepatic steatosis, Semin. Liver Dis. 30, 378-390. 
99. Berk PD, Z. S., Bradbury MW. (2005) Increased hepatocellular uptake of long chain fatty acids occurs by different mechanisms in fatty livers due to obesity or excess ethanol use, contributing to development of steatohepatitis in both settings, Trans Am Clin Climatol Assoc 116, 335-344.

100. Addolorato, G., Capristo, E., Greco, A. V., Stefanini, G. F., and Gasbarrini, G. (1997) Energy expenditure, substrate oxidation, and body composition in subjects with chronic alcoholism: new findings from metabolic assessment, Alcohol Clin Exp Res 21, 962-967.

101. Addolorato, G., Capristo, E., Greco, A. V., Stefanini, G. F., and Gasbarrini, G. (1998) Influence of chronic alcohol abuse on body weight and energy metabolism: is excess ethanol consumption a risk factor for obesity or malnutrition?, J Intern Med 244, 387-395.

102. Kang, L., Chen, X., Sebastian, B. M., Pratt, B. T., Bederman, I. R., Alexander, J. C., Previs, S. F., and Nagy, L. E. (2007) Chronic Ethanol and Triglyceride Turnover in White Adipose Tissue in Rats: Inhibition of the anti-lipolytic action of insulin after chronic ethanol contributes to increased triglyceride degradation, J. Biol. Chem. 282, $28465-28473$.

103. Zhou, S. L., Gordon, R. E., Bradbury, M., Stump, D., Kiang, C. L., and Berk, P. D. (1998) Ethanol up-regulates fatty acid uptake and plasma membrane expression and export of mitochondrial aspartate aminotransferase in HepG2 cells, Hepatology 27, 1064-1074. 
104. Kang, X., Zhong, W., Liu, J., Song, Z., McClain, C. J., Kang, Y. J., and Zhou, Z. (2009) Zinc supplementation reverses alcohol-induced steatosis in mice through reactivating hepatocyte nuclear factor- $4 \alpha$ and peroxisome proliferator-activated receptor- $\alpha$, Hepatology (Hoboken, NJ, U. S.) 50, 1241-1250.

105. Zhong, W., Zhao, Y., Tang, Y., Wei, X., Shi, X., Sun, W., Sun, X., Yin, X., Sun, X., Kim, S., McClain, C. J., Zhang, X., and Zhou, Z. (2012) Chronic alcohol exposure stimulates adipose tissue lipolysis in mice: role of reverse triglyceride transport in the pathogenesis of alcoholic steatosis, Am. J. Pathol. 180, 998-1007.

106. Sun, X., Tang, Y., Tan, X., Li, Q., Zhong, W., Sun, X., Jia, W., McClain, C. J., and Zhou, Z. (2012) Activation of peroxisome proliferator-activated receptor- $\gamma$ by rosiglitazone improves lipid homeostasis at the adipose tissue-liver axis in ethanol-fed mice, Am. J. Physiol. 302, G548-G557.

107. Pouteau, E., Beysen, C., Saad, N., and Turner, S. (2009) Dynamics of adipose tissue development by 2H2O labeling, Methods Mol. Biol. (Totowa, NJ, U. S.) 579, 337-358.

108. Wei, X., Sun, W., Shi, X., Koo, I., Wang, B., Zhang, J., Yin, X., Tang, Y., Bogdanov, B., Kim, S., Zhou, Z., McClain, C., and Zhang, X. (2011) MetSign: a computational platform for high-resolution mass spectrometry-based metabolomics, Anal Chem 83, 7668-7675.

109. Newton MA, N. A., Sarkar D, Ahlquist P. (2004) Detecting differential gene expression with a semiparametric hierarchical mixture method, Biostatistics 5, 155-176.

110. Fisher, R. (1948) Questions and answers \#14, The American Statistician 2, 30-31. 
111. Fisher, R. (1932) Statistical Methods for Research Workers, 4th ed, Edinburgh, Oliver and Boyd.

112. Sumner, L. W., Amberg, A., Barrett, D., Beale, M. H., Beger, R., Daykin, C. A., Fan, T. W. M., Fiehn, O., Goodacre, R., Griffin, J. L., Hankemeier, T., Hardy, N., Harnly, J., Higashi, R., Kopka, J., Lane, A. N., Lindon, J. C., Marriott, P., Nicholls, A. W., Reily, M. D., Thaden, J. J., and Viant, M. R. (2007) Proposed minimum reporting standards for chemical analysis. Chemical Analysis Working Group (CAWG) Metabolomics Standards Initiative (MSI), Metabolomics 3, 211-221.

113. Wang, M.-Y., Grayburn, P., Chen, S., Ravazzola, M., Orci, L., and Unger, R. H. (2008) Adipogenic capacity and the susceptibility to type 2 diabetes and metabolic syndrome, Proc. Natl. Acad. Sci. U. S. A. 105, 6139-6144.

114. Large, V., Peroni, O., Letexier, D., Ray, H., and Beylot, M. (2004) Metabolism of lipids in human white adipocyte, Diabetes Metab. 30, 294-309.

115. Kang, L., and Nagy, L. E. (2006) Chronic ethanol feeding suppresses $\beta$-adrenergic receptor-stimulated lipolysis in adipocytes isolated from epididymal fat, Endocrinology $147,4330-4338$.

116. You, M., and Rogers, C. Q. (2009) Adiponectin: a key adipokine in alcoholic fatty liver, Exp. Biol. Med. (Maywood, NJ, U. S.) 234, 850-859.

117. Greco, A. V., Mingrone, G., Favuzzi, A., Capristo, E., Gniuli, D., Addolorato, G., Brunani, A., Cavagnin, F., and Gasbarrini, G. (2000) Serum leptin levels in post-hepatitis liver cirrhosis, J. Hepatol. 33, 38-42. 
118. Santolaria, F., Perez-Cejas, A., Aleman, M.-R., Gonzalez-Reimers, E., Milena, A., De La Vega, M.-J., Martinez-Riera, A., and Gomez-Rodriguez, M.-A. (2003) Low serum leptin levels and malnutrition in chronic alcohol misusers hospitalized by somatic complications, Alcohol Alcohol. (Oxford, U. K.) 38, 60-66.

119. You, M., Considine, R. V., Leone, T. C., Kelly, D. P., and Crabb, D. W. (2005) Role of adiponectin in the protective action of dietary saturated fat against alcoholic fatty liver in mice, Hepatology (Hoboken, NJ, U. S.) 42, 568-577.

120. Shen, Z., Liang, X., Rogers, C. Q., Rideout, D., and You, M. (2010) Involvement of adiponectin-SIRT1-AMPK signaling in the protective action of rosiglitazone against alcoholic fatty liver in mice, Am. J. Physiol. 298, G364-G374.

121. Tan, X., Sun, X., Li, Q., Zhao, Y., Zhong, W., Sun, X., Jia, W., McClain, C. J., and Zhou, Z. (2012) Leptin deficiency contributes to the pathogenesis of alcoholic fatty liver disease in mice, Am. J. Pathol. 181, 1279-1286.

122. Gao, B., and Bataller, R. (2011) Alcoholic Liver Disease: Pathogenesis and New Therapeutic Targets, Gastroenterology 141, 1572-1585.

123. Janero, D. R., Siuta-Mangano, P., Miller, K. W., and Lane, M. D. (1984) Synthesis, processing, and secretion of hepatic very low density lipoprotein, J. Cell. Biochem. 24, $131-152$.

124. Yao Z, Z. H., Figeys D, Wang Y, Sundaram M. (2012) Microsome-associated lumenal lipid droplets in the regulation of lipoprotein secretion, Curr Opin Lipidol. 
125. Kharbanda, K. K., Todero, S. L., Ward, B. W., Cannella, J. J., III, and Tuma, D. J. (2009) Betaine administration corrects ethanol-induced defective VLDL secretion, Mol. Cell. Biochem. 327, 75-78.

126. Tomita K, A. T., Kitamura N, Nishida J, Tamiya G, et al. (1999) Hepatocyte growth factor leads to recovery from alcohol-induced fatty liver in rats, J Clin Invest 103, 313-320.

127. Day, C. P., and James, O. F. (1998) Steatohepatitis: a tale of two "hits"?, Gastroenterology 114, 842-845.

128. Tilg, H., and Moschen, A. R. (2010) Evolution of inflammation in nonalcoholic fatty liver disease: the multiple parallel hits hypothesis, Hepatology 52, 1836-1846.

129. Cave, M., Deaciuc, I., Mendez, C., Song, Z., Joshi-Barve, S., Barve, S., and McClain, C. (2007) Nonalcoholic fatty liver disease: predisposing factors and the role of nutrition, J Nutr Biochem 18, 184-195.

130. Wahlang, B., Beier, J. I., Clair, H. B., Bellis-Jones, H. J., Falkner, K. C., McClain, C. J., and Cave, M. C. (2013) Toxicant-associated Steatohepatitis, Toxicol. Pathol. 41, 343-360.

131. Tan, M., Schmidt, R. H., Beier, J. I., Watson, W. H., Zhong, H., States, J. C., and Arteel, G. E. (2011) Chronic subhepatotoxic exposure to arsenic enhances hepatic injury caused by high fat diet in mice, Toxicol Appl Pharmacol 257, 356-364.

132. Argos, M., Kalra, T., Rathouz, P. J., Chen, Y., Pierce, B., Parvez, F., Islam, T., Ahmed, A., Rakibuz-Zaman, M., Hasan, R., Sarwar, G., Slavkovich, V., van Geen, A., Graziano, J., and Ahsan, H. (2010) Arsenic exposure from drinking water, and all-cause 
and chronic-disease mortalities in Bangladesh (HEALS): a prospective cohort study, Lancet 376, 252-258.

133. Waalkes, M. P., Liu, J., Chen, H., Xie, Y., Achanzar, W. E., Zhou, Y. S., Cheng, M. L., and Diwan, B. A. (2004) Estrogen signaling in livers of male mice with hepatocellular carcinoma induced by exposure to arsenic in utero, J. Natl. Cancer Inst. 96, 466-474.

134. Mazumder, D. N. (2005) Effect of chronic intake of arsenic-contaminated water on liver, Toxicol. Appl. Pharmacol. 206, 169-175.

135. States, J. C., Singh, A. V., Knudsen, T. B., Rouchka, E. C., Ngalame, N. O., Arteel, G. E., Piao, Y., and Ko, M. S. (2012) Prenatal arsenic exposure alters gene expression in the adult liver to a proinflammatory state contributing to accelerated atherosclerosis, PLoS One 7, e38713.

136. Ralston-Hooper, K., Hopf, A., Oh, C., Zhang, X., Adamec, J., and Sepulveda, M. S. (2008) Development of GCxGC/TOF-MS metabolomics for use in ecotoxicological studies with invertebrates, Aquat Toxicol 88, 48-52.

137. Mohler, R. E., Dombek, K. M., Hoggard, J. C., Pierce, K. M., Young, E. T., and Synovec, R. E. (2007) Comprehensive analysis of yeast metabolite GC x GC-TOFMS data: combining discovery-mode and deconvolution chemometric software, Analyst 132, 756-767.

138. Huang, X., and Regnier, F. E. (2008) Differential metabolomics using stable isotope labeling and two-dimensional gas chromatography with time-of-flight mass spectrometry, Anal. Chem. 80, 107-114. 
139. Kim, S., Koo, I., Jeong, J., Wu, S., Shi, X., and Zhang, X. (2012) Compound identification using partial and semipartial correlations for gas chromatography-mass spectrometry data, Anal. Chem. 84, 6477-6487.

140. Koo, I., Zhang, X., and Kim, S. (2011) Wavelet- and Fourier-transform-based spectrum similarity approaches to compound identification in gas chromatography/mass spectrometry, Anal. Chem. 83, 5631-5638.

141. Wei, X., Shi, X., Koo, I., Kim, S., Schmidt, R. H., Arteel, G. E., Watson, W. H., McClain, C., and Zhang, X. (2013) MetPP: a computational platform for comprehensive two-dimensional gas chromatography time-of-flight mass spectrometry-based metabolomics, Bioinformatics 29, 1786-1792.

142. Castillo, S., Mattila, I., Miettinen, J., Oresic, M., and Hyotylainen, T. (2011) Data analysis tool for comprehensive two-dimensional gas chromatography/time-of-flight mass spectrometry, Anal Chem 83, 3058-3067.

143. Hoggard, J. C., and Synovec, R. E. (2008) Automated resolution of nontarget analyte signals in GC x GC-TOFMS data using parallel factor analysis, Analytical Chemistry 80, 6677-6688.

144. Kozul, C. D., Nomikos, A. P., Hampton, T. H., Warnke, L. A., Gosse, J. A., Davey, J. C., Thorpe, J. E., Jackson, B. P., Ihnat, M. A., and Hamilton, J. W. (2008) Laboratory diet profoundly alters gene expression and confounds genomic analysis in mouse liver and lung, Chem. Biol. Interact. 173, 129-140. 
145. Tong, T., and Zhao, H. (2008) Practical guidelines for assessing power and false discovery rate for a fixed sample size in microarray experiments, Stat Med 27, 1960-1972.

146. Sydor, S., Gu, Y., Schlattjan, M., Bechmann, L. P., Rauen, U., Best, J., Paul, A., Baba, H. A., Sowa, J. P., Gerken, G., and Canbay, A. (2013) Steatosis does not impair liver regeneration after partial hepatectomy, Lab. Invest. 93, 20-30.

147. Shi, X., Wahlang, B., Wei, X., Yin, X., Falkner, K. C., Prough, R. A., Kim, S. H., Mueller, E. G., McClain, C. J., Cave, M., and Zhang, X. (2012) Metabolomic analysis of the effects of polychlorinated biphenyls in nonalcoholic fatty liver disease, J Proteome Res 11, 3805-3815.

148. Lu, S. C. (2013) Glutathione synthesis, Biochim Biophys Acta 1830, 3143-3153.

149. Santra, A., Maiti, A., Chowdhury, A., and Mazumder, D. N. (2000) Oxidative stress in liver of mice exposed to arsenic-contaminated water, Indian J Gastroenterol 19, $112-115$.

150. Ohlsson, L. (2010) Dairy products and plasma cholesterol levels, Food Nutr. Res. 54.

151. Kirpich, I. A., Feng, W., Wang, Y., Liu, Y., Barker, D. F., Barve, S. S., and McClain, C. J. (2012) The type of dietary fat modulates intestinal tight junction integrity, gut permeability, and hepatic toll-like receptor expression in a mouse model of alcoholic liver disease, Alcohol. Clin. Exp. Res. 36, 835-846. 
152. Kono, H., Fujii, H., Asakawa, M., Yamamoto, M., Matsuda, M., Maki, A., and Matsumoto, Y. (2003) Protective effects of medium-chain triglycerides on the liver and gut in rats administered endotoxin, Ann Surg 237, 246-255.

153. Ronis, M. J., Baumgardner, J. N., Sharma, N., Vantrease, J., Ferguson, M., Tong, Y., Wu, X., Cleves, M. A., and Badger, T. M. (2013) Medium chain triglycerides dose-dependently prevent liver pathology in a rat model of non-alcoholic fatty liver disease, Exp Biol Med (Maywood) 238, 151-162.

154. Gupta, S., Knight, A. G., Keller, J. N., and Bruce-Keller, A. J. (2012) Saturated long-chain fatty acids activate inflammatory signaling in astrocytes, Journal of neurochemistry 120, 1060-1071.

155. Huang, S., Rutkowsky, J. M., Snodgrass, R. G., Ono-Moore, K. D., Schneider, D. A., Newman, J. W., Adams, S. H., and Hwang, D. H. (2012) Saturated fatty acids activate TLR-mediated proinflammatory signaling pathways, Journal of lipid research 53, 2002-2013.

156. Murumalla, R. K., Gunasekaran, M. K., Padhan, J. K., Bencharif, K., Gence, L., Festy, F., Cesari, M., Roche, R., and Hoareau, L. (2012) Fatty acids do not pay the toll: effect of SFA and PUFA on human adipose tissue and mature adipocytes inflammation, Lipids in health and disease 11, 175.

157. Harvey, K. A., Walker, C. L., Pavlina, T. M., Xu, Z., Zaloga, G. P., and Siddiqui, R. A. (2010) Long-chain saturated fatty acids induce pro-inflammatory responses and impact endothelial cell growth, Clin Nutr 29, 492-500. 
158. Begriche, K., Massart, J., Robin, M. A., Bonnet, F., and Fromenty, B. (2013) Mitochondrial adaptations and dysfunctions in nonalcoholic fatty liver disease, Hepatology.

159. Joseloff, E., Sha, W., Bell, S. C., Wetmore, D. R., Lawton, K. A., Milburn, M. V., Ryals, J. A., Guo, L., and Muhlebach, M. S. (2013) Serum metabolomics indicate altered cellular energy metabolism in children with cystic fibrosis, Pediatric pulmonology.

160. Ikejima, K., Iimuro, Y., Forman, D. T., and Thurman, R. G. (1996) A diet containing glycine improves survival in endotoxin shock in the rat, Am. J. Physiol. 271, G97-103.

161. Rose, M. L., Germolec, D., Arteel, G. E., Schoonhoven, R., and Thurman, R. G. (1997) Dietary glycine prevents increases in hepatocyte proliferation caused by the peroxisome proliferator WY-14,643, Chem. Res. Toxicol. 10, 1198-1204.

162. Ikejima, K., Qu, W., Stachlewitz, R. F., and Thurman, R. G. (1997) Kupffer cells contain a glycine-gated chloride channel, Am. J. Physiol. 272, G1581-1586.

163. Wheeler, M. D., Ikejema, K., Enomoto, N., Stacklewitz, R. F., Seabra, V., Zhong, Z., Yin, M., Schemmer, P., Rose, M. L., Rusyn, I., Bradford, B., and Thurman, R. G. (1999) Glycine: a new anti-inflammatory immunonutrient, Cell. Mol. Life Sci. 56, 843-856.

164. Leoncini, R., Pagani, R., Marinello, E., and Keleti, T. (1989) Double inhibition of L-threonine dehydratase by aminothiols, Biochim. Biophys. Acta 994, 52-58.

165. Manohar, R., and Appaji Rao, N. (1984) Identification of active-site residues of sheep liver serine hydroxymethyltransferase, Biochem. J. 224, 703-707. 
166. Gavilanes, F., Peterson, D., Bullis, B., and Schirch, L. (1983) Structure and reactivity of cysteine residues in mitochondrial serine hydroxymethyltransferase, J. Biol. Chem. 258, 13155-13159.

167. Huang, C., Yin, Q., Zhu, W., Yang, Y., Wang, X., Qian, X., and Xu, Y. (2011) Highly selective fluorescent probe for vicinal-dithiol-containing proteins and in situ imaging in living cells, Angew. Chem. Int. Ed. Engl. 50, 7551-7556.

168. Kirpich, I. A., and McClain, C. J. (2012) Probiotics in the treatment of the liver diseases, Journal of the American College of Nutrition 31, 14-23.

169. Wang, Y., Kirpich, I., Liu, Y., Ma, Z., Barve, S., McClain, C. J., and Feng, W. (2011) Lactobacillus rhamnosus GG treatment potentiates intestinal hypoxia-inducible factor, promotes intestinal integrity and ameliorates alcohol-induced liver injury, Am. J. Pathol. 179, 2866-2875.

170. Forsyth, C. B., Farhadi, A., Jakate, S. M., Tang, Y., Shaikh, M., and Keshavarzian, A. (2009) Lactobacillus GG treatment ameliorates alcohol-induced intestinal oxidative stress, gut leakiness, and liver injury in a rat model of alcoholic steatohepatitis, Alcohol (N. Y., NY, U. S.) 43, 163-172.

171. Kelishadi, R., Farajian, S., and Mirlohi, M. (2013) Probiotics as a Novel Treatment for Non-Alcoholic Fatty Liver Disease; A Systematic Review on the Current Evidences, Hepatitis Monthly 13.

172. Wang, Y., Liu, Y., Kirpich, I., Ma, Z., Wang, C., Zhang, M., Suttles, J., McClain, C., and Feng, W. (2013) Lactobacillus rhamnosus GG reduces hepatic TNF $\alpha$ production and inflammation in chronic alcohol-induced liver injury, J. Nutr. Biochem. 24, 1609-1615. 
173. Wang, Y., Liu, Y., Sidhu, A., Ma, Z., McClain, C., and Feng, W. (2012) Lactobacillus rhamnosus GG culture supernatant ameliorates acute alcohol-induced intestinal permeability and liver injury, Am. J. Physiol. 303, G32-G41.

174. Zhang, M., Wang, C., Wang, C., Zhao, H., Zhao, C., Wang, Y., McClain, C., and Feng, W. (2014) Enhanced AMPK phosphorylation contributes to the beneficial effects of Lactobacillus rhamnosus GG supernatant on chronic alcohol-induced fatty liver disease, J Nutr Biochem In press.

175. Ralston-Hooper, K., Hopf, A., Oh, C., Zhang, X., Adamec, J., and Sepulveda, M. S. (2008) Development of GCxGC/TOF-MS metabolomics for use in ecotoxicological studies with invertebrates, Aquat Toxicol 88, 48-52.

176. Liu, S. M., Miller, D. M., and Roberts, R. F. (2011) Cloning of genes encoding colicin E2 in Lactococcus lactis subspecies lactis and evaluation of the colicin-producing transformants as inhibitors of Escherichia coli O157:H7 during milk fermentation, Journal of Dairy Science 94, 1146-1154.

177. Godsey, H. S., Oviatt, C. G., Miller, D. M., and Chan, M. A. (2011) Stratigraphy and chronology of offshore to nearshore deposits associated with the Provo shoreline, Pleistocene Lake Bonneville, Utah, Palaeogeogr Palaeocl 310, 442-450.

178. Koo, I., Zhang, X., and Kim, S. (2011) Wavelet- and Fourier-Transform-Based Spectrum Similarity Approaches to Compound Identification in Gas Chromatography/Mass Spectrometry, Analytical Chemistry 83, 5631-5638.

179. Zhang, J., Fang, A. Q., Wang, B., Kim, S. H., Bogdanov, B., Zhou, Z. X., McClain, C., and Zhang, X. (2011) iMatch: A retention index tool for analysis of gas 
chromatography-mass spectrometry data, Journal of Chromatography A 1218, $6522-6530$.

180.Rao, M. S., and Reddy, J. K. (2001) Peroxisomal $\beta$-oxidation and steatohepatitis, Semin. Liver Dis. 21, 43-55.

181. Vila, L., Roglans, N., Alegret, M., Sanchez, R. M., Vazquez-Carrera, M., and Laguna, J. C. (2008) Suppressor of cytokine signaling-3 (SOCS-3) and a deficit of serine/threonine (Ser/Thr) phosphoproteins involved in leptin transduction mediate the effect of fructose on rat liver lipid metabolism, Hepatology (Hoboken, NJ, U. S.) 48, 1506-1516.

182. Charlton, M. (2006) Branched-chain amino acid enriched supplements as therapy for liver disease, J. Nutr. 136, 295S-298S.

183. Calvey, H., Davis, M., and Williams, R. (1985) Controlled trial of nutritional supplementation, with and without branched chain amino acid enrichment, in treatment of acute alcoholic hepatitis, J Hepatol 1, 141-151.

184. Chen, P., Loomba, R., Torralba, M., DePew, J., Nelson, K. E., Tan, J., Embree, M., Zengler, K., Starkel, P., Pijkeren, J.-P. v., Ho, S. B., Bajaj, J. S., Mutlu, E. A., Keshavarzian, A., Tsukamoto, H., Fouts, D. E., and Schnabl, B. (2014) Supplementation of Saturated Long-chain Fatty Acids Maintains Intestinal Eubiosis and Reduces Ethanol-induced Liver Injury in Mice, Gastroenterology.

185. Aggarwal, S., Suzuki, T., Taylor, W. L., Bhargava, A., and Rao, R. K. (2011) Contrasting effects of ERK on tight junction integrity in differentiated and under-differentiated Caco-2 cell monolayers, Biochem. J. 433, 51-63. 
186. Bull-Otterson, L., Feng, W., Kirpich, I., Wang, Y., Qin, X., Liu, Y., Gobejishvili, L., Joshi-Barve, S., Ayvaz, T., Petrosino, J., Kong, M., Barker, D., McClain, C., and Barve, S. (2013) Metagenomic analyses of alcohol induced pathogenic alterations in the intestinal microbiome and the effect of Lactobacillus rhamnosus GG treatment, PLoS ONE 8, e53028. 


\section{APPENDIX \\ LIST OF ABBREVIATIONS}

AF: Alcohol-containing Diet

AFLD: Alcoholic Fatty Liver Disease

ALT: Plasma Alanine Aminotransferase

AMPKa: Adenosine Monophosphate-activated Protein Kinase- $\alpha$

AUC: Area Under Curve

BCAA: Branched-chain Amino Acid

BHT: Butylatedhydroxytoluene

CD: Control Diet

CID: Collision-induced Dissociation

DI-MS: Direct Infusion Mass Spectrometry

DI-nESI: Direct Infusion Chip-based Nano-electrospray Ionization

eWAT: epididymal White Adipose Tissue

FDR: False Discovery Rate

FFA: Free Fatty Acids

FLD: Fatty Liver Disease

FN: False-negative

FP: False-positive

FTICR-MS: Fourier Transform Ion Cyclotron Resonance Mass Spectrometry 
GC×GC-TOF MS: Two-dimensional Gas Chromatography Time-of-flight Mass

Spectrometry

GC-MS: Gas Chromatography Mass Spectrometry

GMM: Gaussian Mixture Model

GSH: Glutathione (GSH)

HFD: High Fat Diet

HILIC: Hydrophobic Interaction Liquid Chromatography

HMDB: Human Metabolome Database

HSL: Hormone Sensitive Lipase

IPA: Ingenuity Pathway Analysis

KEGG: Kyoto Encyclopedia of Genes and Genomes

LCFA: Long Chain Fatty Acid

LC-MS: Liquid Chromatography Mass Spectrometry

LGG: Lactobacillus Rhamnosus GG

LIT: Linear Ion Trap

LPS: Lipopolysaccharide

LTQ-FTICR MS: Linear Trap Quadruple - Fourier Transform Ion Cyclotron Mass

Spectrometer

M: Molar

mg: milligram

mL: milliliter

mmol: millimole

MS: Mass Spectrometry

NAFLD: Non-alcoholic Fatty Liver Disease

NASH: Non-alcoholic Steatohepatitis 
NCE: Normalized Collision Energy

nESI: Nano-electrospray Ionization

NMR: Nuclear Magnetic Resonance

OCT: Optimal Cutting Temperature

PCB: Polychlorinated Biphenyls

PF: Isocaloric Control Diet

PPB: Parts Per Billion

PPM: Parts Per Million

PPV: Positive Predictive Value

PTEN: Phosphatase and Tensin Homolog

ROC: Receiver Operating Characteristic

ROS: Reactive Oxygen Species

RP-LC: Reverse Phase Liquid Chromatography

RP: Resolving Power

RSD: Relative Standard Deviation

SCE: Strong Cation Exchange

SCFA: Short Chain Fatty Acids

SIAM: Stable Isotope Assisted Metabolomics

SIR: Selected Ion Recording

SPF: Second-order Polynomial Fitting

SRM: Slected Reaction Monitoring

sWAT: Subcutaneous White Adipose Tissue

TGs: Triacylglycerols

TP: True-positive

TPR: True-positive Rate 
VLDL: Very Low-density Lipoproteins

WAT: White Adipose Tissue

\section{ACS Publications Title: \\ Most Trusted. Most Cited. Most Read.}

Author:

Publication

Publisher:

Date:

Copyright @ 2012, American Chemical Society
Metabolomic Analysis of the Effects of Polychlorinated Biphenyls in Nonalcoholic Fatty Liver Disease

Xue Shi, Banrida Wahlang, Xiaoli Wei, et al
Logged in as:

Xue Shi

LOGOUT

PERMISSION/LICENSE IS GRANTED FOR YOUR ORDER AT NO CHARGE

This type of permission/license, instead of the standard Terms \& Conditions, is sent to you because no fee is being charged for your order. Please note the following:

- Permission is granted for your request in both print and electronic formats, and translations.

- If figures and/or tables were requested, they may be adapted or used in part.

- Please print this page for your records and send a copy of it to your publisher/graduate school.

- Appropriate credit for the requested material should be given as follows: "Reprinted (adapted) with permission from (COMPLETE REFERENCE CITATION). Copyright (YEAR) American Chemical Society." Insert appropriate information in place of the capitalized words.

- One-time permission is granted only for the use specified in your request. No additional uses are granted (such as derivative works or other editions). For any other uses, please submit a new request. 
ACSPublications Title:

Most Trusted. Most Cited. Most Read.

The:

Metabolomic Analysis of the Effects of Chronic Arsenic Exposure in a Mouse Model of Diet-Induced Fatty Liver Disease

Author: Xue Shi, Xiaoli Wei, Imhoi Koo, et al

Publication: Journal of Proteome Research

Publisher: American Chemical Society

Date: $\quad$ Feb 1, 2014

Copyright (9) 2014, American Chemical Society

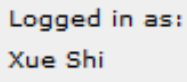

LOGOUT

\section{PERMISSION/LICENSE IS GRANTED FOR YOUR ORDER AT NO CHARGE}

This type of permission/license, instead of the standard Terms \& Conditions, is sent to you because no fee is being charged for your order. Please note the following:

- Permission is granted for your request in both print and electronic formats, and translations.

- If figures and/or tables were requested, they may be adapted or used in part.

- Please print this page for your records and send a copy of it to your publisher/graduate school.

- Appropriate credit for the requested material should be given as follows: "Reprinted (adapted) with permission from (COMPLETE REFERENCE CITATION). Copyright (YEAR) American Chemical Society." Insert appropriate information in place of the capitalized words.

- One-time permission is granted only for the use specified in your request. No additional uses are granted (such as derivative works or other editions). For any other uses, please submit a new request. 


\section{CURRICULUM VITAE}

\section{Xue Shi}

Tel: 502-619-9978

Email: xueshisx@gmail.com

\section{SKILLS}

\section{Analytical Instrumentation:}

GC-MS, GC-HRT MS, GC×GC-TOF MS, Nanomate-LTQ-FTICR MS, LC/UV/Vis, LC-MS, UPLC-MS, CE-ED, AAS

\section{Software Packages:}

Instrument control: ChromaTOF, ChemStation, Xcalibur, Chromeleon

Data mining:

ChromaTOF, Mass Frontier, Ingenuity Pathway Analysis (IPA), ChemBioDraw, MetPP, MetSign, MetaCore, MetaDrug, MZmine, NIST MS

\section{RESEARCH EXPERIENCE}

\section{Ph.D. Thesis Research}

\section{University of Louisville}

Metabolomic analysis of the effects of chronic arsenic exposure in a mouse model of diet-induced fatty liver disease by using GC×GC-TOF MS

- Pegasus 4D GC×GC-TOF MS instrument trouble shooting and maintenance

- Optimized extraction, derivatization method and GCxGC-TOF MS method development for biological sample

- Developed analytical and assisted developed bioinformatics platform MetPP for biomarker discovery

Developing HILIC $\times$ RP-MS platform for metabolomics analysis 
- Dionex 3000 UPLC/UV/Vis and Agilent nanoflow 1200 instrument set-up and trouble shooting

- Optimized HILIC-MS and RP-MS separation of mouse liver extract

- Assisted bioinformaticians to develop MetSign software and optimize parameters for quantitative analysis

Applied direct infusion nanomate FTICR MS for biomarker discovery

- Developed protocol for analysis of metabolites in mouse liver

- Assisted the development of MetSign software for FTICR MS data analysis

Applied AAS for analysis of $\mathrm{Zn}, \mathrm{Fe}, \mathrm{Cu}, \mathrm{Mn}, \mathrm{Mg}$ concentration in liver tissue

- Developed analytical protocols for analysis of metal ions in mouse liver tissue

\section{M.S. Thesis Research}

East China Normal University

Analysis of additives in cosmetic and food, active ingredient in Chinese Medicine by capillary electrophoresis with electrochemical detection

- CE-ED instrument set-up and trouble shooting

- Optimize separation and study linearity, recovery, repeatability, detection limit for the ingredients, then used for application

\section{WORKING EXPERIENCE}

\section{Coldstream Laboratories, Inc. A R\&D Associate Scientist $\quad 12 / 2013$ to now}

Developing, establishing, validation and transferring analytical methods including HPLC, GC;

Reviewing, analyzing data and information for technical correctness, accuracy, interpretation and

evaluation of results;

Write and review Standard Operating Procedures, analytical deviations and laboratory investigations.

\section{EDUCATION}

\section{PhD candidate: University of Louisville}

Analytical Chemistry, Advisor: Dr. Xiang Zhang

M.S.: East China Normal University

06/2009

Analytical Chemistry, Advisor: Dr. Jiannong Ye

\section{B.S.: Shandong Normal University}

$06 / 2006$

Chemical Engineering and Materials Science 


\section{HONORS AND FELLOWSHIP}

2013 Kirkbright Bursary Award, Association of British Spectroscopists

$02 / 2013$

Graduate Dean's Citation, Univesity of Louisville

$11 / 2014,12 / 2012$

Research Assistantship, University of Louisville

08/2010-07/2013

Teaching Assistantship, University of Louisville

08/2009-07/2010

Excellent Graduate Student Scholarship, East China Normal University

$10 / 2008$

Best Undergraduate Dissertation, Shandong Normal University

$06 / 2006$

Excellent League Member, Shandong Normal University

03/2005-03/2006

Excellent Student Scholarship, Shandong Normal University

09/2004-09/2009

\section{$\underline{\text { TEACHING EXPERIENCE }}$}

Teaching Assistant, University of Louisville

08/2009-07/2010, 08/2013-12/2013

- Supervised analytical chemistry lab 207/208 including titration, spectrometer analysis, grading for prelab and lab report, held weekly office hour

- Proctored and graded for General Chemistry 201/202 and 101

Teaching Assistant, East China Normal University

09/2007-01/2008

- Instructed student in GC/LC lab

Instructed undergraduate student and new graduate student on lab techniques and assisted student with the design and execution of research project and bachelor thesis writing

\section{PROFESSIONAL TRANINING EXPERIENCE}

LECO Pegasus 4D GC×GC-TOFMS ChromaTOF Training

$04 / 2010$

\section{$\underline{\text { SELECTED PUBLICATIONS }}$}

1) Shi, X.; Hukill B.; Laulhe S.; Nantz M.; Zhang,X. Differential metabolomics study using stable isotope labeling and GC×GC-TOFMS. Anal. Chem., In preparation.

2) Shi, X.; Yin, X.; Wei,X.; Koo, I; McClain, C.; Wenke Feng; Zhang,X. Hepatic and fecal metabolomic analysis of the effects of Lactobacillus Rhamnosus GG on alcoholic fatty liver disease.. J. Proteome Res. In Revision. 
3) Wei, X.; Shi, X.; Kim, S. H.; Patrick J.S.;Binkley J.;Kong M.; McClain, C.; Zhang, X. Data Dependent Peak Model Based Spectrum Deconvolution for Analysis of High Resolution LC-MS Data. Anal. Chem., 2014, 86 (4): 2156.

4) Koo I.;Wei, X.; Shi, X.; Zhou Z.; Kim, S. H.; Zhang, X.Constructing metabolic association networks using high-dimensional mass spectrometry data. Chemometrics and Intelligent Laboratory Systems, 2014, 138:193.

5) Koo I.; Shi, X.; Kim, S. H.; Zhang, X. iMatch2: Compound Identification Using Retention Index for Analysis of Gas Chromatography-Mass Spectrometry Data. JCA, 2014, 1337:202.

6) Shi, X.; Yin, X.; Zhang, X. Analysis of mouse liver metabolites on GC $\times$ GC-TOF MS. Methods Mol Biol., 2014, 1198: 99.

7) Shi, X.; Wei,X.; Koo, I, Yin, X.; Schmidt, R.; Kim,S.; McClain, C.; Arteel G; Watson W; Zhang,X. Metabolomic analysis of the effects of chronic arsenic exposure in a mouse model of diet-induced fatty liver disease. J. Proteome Res., 2014, 13 (2): 547.

8) Wei, X.; Shi, X.; Merrick M.; Willis P.; Alonso D.; Zhang, X. A Method of Aligning Peak Lists Generated by Gas Chromatography High-resolution Mass Spectrometry. Analyst, 2013, 138:5453

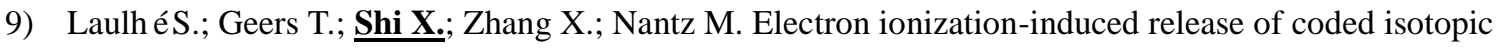
reporter ions in an $\mathrm{m} / \mathrm{z}$ zone of minimal interference for quantifiable, multiplexed GC-MS analyses. Analytical Methods, 2013, 5: 4701.

10) Shi, X.; Wei, X.; Tang, Y.; Sun, W.; Yin, X.; Sun, X.; Bogdanov, B.; Kim, K.; McClain, C.; Zhou, Z.; Zhang, X. Chronic Alcohol Exposure Disturbs Lipid Homeostasis at the Adipose Tissue-Liver Axis in Mice: Analysis of Triacylglycerols Using High-Resolution Mass Spectrometry in Combination with In Vivo Metabolite Deuterium Labeling. PLoS One. 2013, 8(2): e55382.

11) Jeong, J.; Zhang, X; Shi, X.; Kim, S.; Shen, C. An efficient post-hoc integration method improving peak alignment of metabolomics data from GCxGC/TOF-MS. BMC Bioinformatics. 2013, $14: 123$.

12) Wei, X.; Shi, X.; Koo, I.; Kim, S. H.; McClain, C.; Zhang, X. MetPP: a computational platform for comprehensive two-dimensional gas chromatography time-of-flight mass spectrometry-based metabolomics. Bioinformatics. 2013, 29(14): 1786-1792 .

13) Shi, X.; Wahlang, B.; Wei, X.; Yin, X.; Falkner, K.; Prough, R.; Kim, S.; McClain, C.; Cave, M.; 
Zhang, X. Metabolomic analysis of the effects of polychlorinated biphenyls in non-alcoholic fatty liver disease. J. Proteome Res. 2012, 11, 3805-3815.

14) Wei, X.; Shi, X.; Kim, S. H.; Zhang, L.; Patrick, J. S.; Binkley, J.; McClain, C.; Zhang, X. A data pre-processing method for liquid chromatography mass spectrometry-based metabolomics. Anal. Chem. 2012, 84, 7963-7971.

15) Kim, S.; Koo, I.; Jeong, J.; Wu, S.; Shi, X.; Zhang, X. Compound identification using partial and semi-partial correlations for gas chromatography mass spectrometry data. Anal. Chem. 2012, 84, 6477-6487.

16) Jeong, J.; Shi, X.; Zhang, X.; Kim, S.; Shen, C. Model-based peak alignment of metabolomics profiling from comprehensive two-dimensional gas chromatography mass spectrometry. BMC Bioinformatics 2012, 13:27.

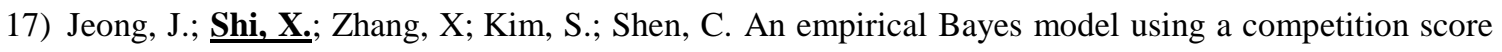
for metabolite identification in gas chromatography mass spectrometry. BMC Bioinformatics 2011, $12: 392$.

18) Wei, X.; Sun, W.; Shi, X.; Koo, I.; Wang, B.; Zhang, J.; Yin, X.; Tang, Y.; Bogdanov, B.; Kim, S. H.; Zhou, Z.; McClain, C. J.; Zhang, X. MetSign: A computational platform for high-resolution mass spectrometry-based metabolomics. Anal. Chem. 2011, 83, 7668-7675.

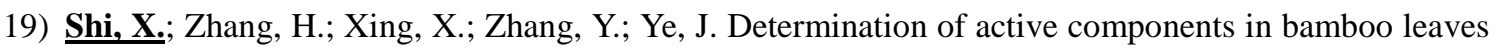
and bamboo-leaf tea by capillary electrophoresis with electrochemical detection. J. Anal. Sci. 2009, 25 (2), 143-147.

\section{SELECTED PRESENTATIONS}

1) Shi X.; Wei X.; Koo I.; Schmidt R.; Yin X.; Kim S.; Vaughn A.; Arteel G.; McClain C.; Watson W.; Zhang X. Metabolomics analysis of chronic arsenic exposure in a mouse model of diet-induced fatty liver disease. PITTCON Conference \& Expo 2013, Philadephia, PA, Mar. 17-21, 2013.

2) Wei X.; Shi X.; Koo I.; Kim S.; McClain C.; Zhang X. A Computational Platform for Comprehensive Two-dimensional Gas Chromatography-Time of Flight Mass Spectrometry-Based Metabolomics Profiling. PITTCON Conference \& Expo 2013, Philadephia, PA, Mar. 17-21, 2013.

3) Wei X.; Shi X.; Kim S.; Zhang L.; Patrrick J.; Binkley J.; McClain C.; Zhang X. A Computational 
Platform for High Resolution Mass Spectrometry-Based and Liquid Chromatography Mass Spectrometry-Based Metabolomics. PITTCON Conference \& Expo 2013, Oral, Philadephia, PA, Mar. 17-21, 2013.

4) Laulhé S.; Shi X.; Geers T.; Zhang X.; and Nantz M. Quantifiable Multiplexed GC-MS Analysis of Carbonyl and Amine Metabolites Using the Electron-Induced Cleavage of an Isotopic Reporter Tag. Poster No. 13, 15th Annual IMD3 Symposium, Louisville, KY; March 12, 2013.

5) Laulhé S.; Geers T.; Shi X.; Zhang X.; and Nantz M. Multiplexed Metabolite Quantitation Using an Isotope Coding Strategy for GC-MS Analysis. Oral, SERMACS, Raleigh, NC; November 16, 2012.

6) Zhang, X.; Shi, X.; Zhang, L.; Slek, K.; Wahlang, B.; Wei, X.; Koo, I.; Patrick, J.; Binkley, J.; Kim, S.; McClain, C.; Cave, M. A Comprehensive Approach to Disease Biomarker Discovery. 60th ASMS Conference on Mass Spectrometry and Allied Topics, Vancouver, BC, CA. May 20 - 24, 2012.

7) Wahlang, B.; Shi, X.; Zhang, X.; Falkner, K.C.; McClain, C.J.; Prough, R.; Cave, M. The metabolic effects of PCB 153 depend on nutrient interactions in obesity/nonalcoholic fatty liver disease. Digestive Disease Week 2012. San Diego, CA. May 19 - 22, 2012.

8) Shi, X.; Wang, B.; Zhang, J.; Wei, X.; Kim, S.; Kirpich, I.; Feng, W.; McClain, C.; Zhang, X. Metabolomics analysis of alcohol on mice fed with unsaturated fat diet using GC $\times$ GC-MS. 59th ASMS Conference on Mass Spectrometry and Allied Topics, Denver, CO. June 5 - 9, 2011. 